\title{
Resource Allocation and Interference Management in Heterogeneous Wireless Networks
}

\author{
by \\ Ali Y. Al-Zahrani \\ A Thesis submitted to \\ the Faculty of Graduate and Postdoctoral Affairs \\ in partial fulfilment of \\ the requirements for the degree of \\ Doctor of Philosophy in Electrical and Computer Engineering \\ Ottawa-Carleton Institute for \\ Electrical and Computer Engineering
}

Department of Systems and Computer Engineering

Carleton University

Ottawa, Ontario, Canada

January 2015

Copyright (C)

2015 - Ali Y. Al-Zahrani 


\section{Abstract}

Heterogeneous networks (HetNet) are a promising solution to improve network performance in terms of spectrum efficiency and energy efficiency. Nevertheless, HetNets suffer from two main sources of interference: mutual interference between macrocells and small cells (called cross-layer interference), as well as inter-cell interference among small cells themselves (called co-layer interference). In this thesis, we study the resource allocation and interference issues of HetNets. First, in HetNet systems with a moderate number of small cells, we integrate two popular approaches: spectrum avoidance and spectrum sharing, using optimization and game theory. In this solution, small base stations (SBSs) opportunistically avoid the parts of the spectrum that are used by macro BS (MBS), thereby controlling cross-layer interference, while the co-layer interference is controlled using a spectrum sharing technique. We then exploit recent advances in the mean-field game theory in order to control the co-layer interference between a large number of small cells. Meanwhile, a spectrum avoidance technique is applied to control the cross-layer interference. Next, we design a full spectrum sharing technique based on the mean-field game theory for interference management in hyper dense HetNet systems. The joint cross-layer and co-layer interference management issue is formulated as two nested problems, which are solved via distributed algorithms. Furthermore, tools from the optimization theory are employed to enhance the performance of cell edge users in open access HetNets. Simulation results are presented to show the effectiveness of the proposed schemes. 
To my parents: Yahya Al-Zahrani \& Safyah Al-Zahrani 


\section{Acknowledgments}

I am so grateful to God (Allah) for his uncountable bounties on me including the ability to perform this research and to write this thesis. I am deeply indebted to many people for their help during the multiple phases of this work.

My deep and sincere appreciation goes to my supervisor: Professor Richard Yu for all the hours and days of help, guidance, and intellectual discussion. Thanks for your continuous support and kind cooperation, catching my misunderstandings and vague wordings and thereby greatly improving the quality of this thesis.

I am infinitely grateful to my mother for her overwhelming love, continuous care as well as honest prayers for me. My deceased father is a source of inspiration for me. His strive for the whole family will not be forgotten. I am incredibly lucky for being their son. This thesis is dedicated to them.

The Lord has blessed me with a wonderful family to the point that no words are sufficient to express my gratitude and love for them. I am very grateful to my wife: Fatmah AlZahrani who was always beside me, and has encouraged me with infinite support to accomplish this achievement. My children: Yahya, Reem, Raneem and Yonis and their mother are indeed my special people who make my life very meaningful and wonderful. Time constraints and struggling to complete this work made me unable to give them all what they deserve, and hence I will never forget their understanding and patience.

I would like also to acknowledge my brothers who played a great role in my 
education. Special thanks goes to my sisters who supported me throughout my life.

Finally, my colleagues in the lab deserve my appreciation for incredible cooperation, many useful comments on the thesis as well as sharing fun times with them. 


\section{Table of Contents}

Abstract $\quad$ ii

Acknowledgments $\quad$ iv

Table of Contents $\quad$ vi

List of Figures $\quad$ xi

Nomenclature $\quad$ xiv

1 Introduction 1

1.1 Challenges Facing HetNets . . . . . . . . . . . . . . . . 2

1.2 Problem Statement . . . . . . . . . . . . . . . 5

1.3 Main Contributions and Thesis Organization . . . . . . . . . 6

1.3.1 Thesis Outline and List of Publications . . . . . . . . . . 8

2 Preliminaries and Background $\quad 11$

2.1 Channel Model ... . . . . . . . . . . . . . . 11

2.2 Channel Adaptation . . . . . . . . . . . . . . . . . 15

2.3 Mathematical Tools . . . . . . . . . . . . . . . . . 16

2.3.1 Game Theory . . . . . . . . . . . . . . 17

2.3.2 Mean-Field Game Theory . . . . . . . . . . . . . . . . . 19

2.3.3 Mean Field Approximation (MFA) . . . . . . . . . . . . . . . 22 
2.3.4 Hungarian Algorithm . . . . . . . . . . . . . . . . . . . . 23

3 Resource Allocation and Interference Management in HetNets: A Literature Review $\quad 26$

3.1 General Resource Allocation and Interference Management Schemes . 27

3.2 Game Theoretic Approaches for Resource Allocation and Interference Management . . . . . . . . . . . . . . . . . . . . . . . 31

4 Resource Allocation and Interference Management in HetNets with Moderate Number of Small Cells $\quad 35$

4.1 System Description . . . . . . . . . . . . . . . . . . 37

4.2 Macro-BS Overlay Optimization Problem . . . . . . . . . . . . . 39

4.2 .1 Problem Formulation . . . . . . . . . . . . . . . . . . . 39

4.2 .2 Overlay Algorithm in MBS . . . . . . . . . . . . . . . . . 41

4.3 Formulation of a Non-Cooperative Game . . . . . . . . . . . 45

4.3.1 Non-Cooperative Game Among SBSs . . . . . . . . . . . . . 46

4.3 .2 Utility Function . . . . . . . . . . . . . . . . . . 47

4.3.3 Equilibrium Analysis: Existence and Uniqueness . . . . . . . 52

4.3.4 Game Equilibrium Outcome . . . . . . . . . . . . . . 55

4.3.5 Distributed Power Control Algorithm . . . . . . . . . . . . 57

4.3.6 Subchannels' Maximum Power Allocation . . . . . . . . . 59

4.4 Simulation Results and Discussions _. . . . . . . . . . . . . . 60

$4.4 .1 \quad$ Organized Deployment . . . . . . . . . . . . . . 62

$4.4 .2 \quad$ Spontaneous Deployment . . . . . . . . . . . . . . . 65

4.5 Conclusions . . . . . . . . . . . . . . . . . . . . . . 69

5 Resource Allocation and Interference Management in HetNets with a Large Number of Small Cells $\quad 72$ 
5.1 System Description . . . . . . . . . . . . . . . . . . . 74

5.2 Formulation of the Macro-BS Optimization Problem . . . . . . . 75

5.3 Formulation of the Mean-Field Game among Small BSs . . . . . . . 76

5.3.1 Estimation of the Interference Channels' Average $\bar{h}_{-n m} \ldots$. . 79

5.3.2 Conjecture of the Interferers' Transmit Power Average $\bar{p}_{-n m}$. 80

5.3 .3 Utility Functions . . . . . . . . . . . . . . . . . . . . . . . 81

5.3.4 Decentralized Solution . . . . . . . . . . . . . . . . . 81

5.4 Simulation Results and Discussions _. . . . . . . . . . . . 83

5.4.1 Random Network Topology _. . . . . . . . . . . . . . . 83

5.4 .2 Organized Network Topology _. . . . . . . . . . . 86

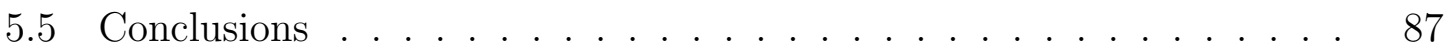

6 Resource Allocation and Interference Management Based on Full Spectrum Sharing for Hyper-Dense HetNets 89

6.1 System Description and Problem Formulation . . . . . . . . . . . . 91

6.1 .1 Overlay Problem Formulation $\ldots \ldots \ldots$

6.1.2 Underlay Problem Formulation . . . . . . . . . . . . . 95

6.2 Underlay Game Treatment . . . . . . . . . . . . . . . . . . . 96

6.2.1 System Decoupling Using Mean-Field Theory _ . . . . . . . 97

6.2.2 Game Outcome and Condition . . . . . . . . . . . . . . 98

6.2.3 Underlay Interference Control Algorithm . . . . . . . . . . 100

6.3 Overlay Problem Treatment . . . . . . . . . . . . . . . . . . . 101

6.3.1 Overlay Interference Control Algorithm . . . . . . . . . . 105

6.3 .2 Selecting $p_{m}^{\text {initial } \ldots \ldots \ldots \ldots ~ \ldots ~ \ldots ~ \ldots ~ \ldots ~ . ~ . ~ . ~ . ~ . ~} 106$

6.4 Simulation Results and Discussions _. . . . . . . . . . . . 113

6.4.1 Planned Deployment . . . . . . . . . . . . . . . . . . 114

6.4 .2 Random Deployment . . . . . . . . . . . . . . . . . . 118 
6.5 Conclusions . . . . . . . . . . . . . . . . . . . 120

7 Enhancing Cell Edge Users Performance in Open Access HetNets 123

7.1 System Description . . . . . . . . . . . . . . . . . 125

7.2 Fully Distributed Scheduling Schemes . . . . . . . . . . . . . . 128

7.2.1 Network-Centric Objective (NCO) _ . . . . . . . . . . . 129

7.2.2 Cross Layer-Based Scheduling Schemes _ . . . . . . . . . 130

7.3 Generalized Formulation . . . . . . . . . . . . . . . . . . . 132

7.4 Simulation Results and Discussions . . . . . . . . . . . . . . 134

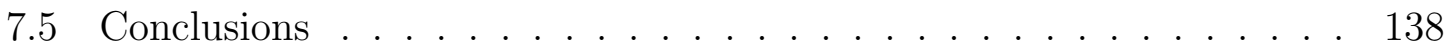

8 Conclusion and Research Directions 142

8.1 Extensions to the Proposed Research . . . . . . . . . . . . . . 144

8.1.1 Priority-Less Schemes . . . . . . . . . . . . . . . . . 144

8.1.2 Incorporating a Medium Access Control (MAC) Protocol . . . 144

8.1.3 Stochastic Dynamic Games . . . . . . . . . . . . . . . 145

8.1.4 Device-to-Device Communication . . . . . . . . . . . 145

8.2 Relevant Research Directions . . . . . . . . . . . . . . . 146

8.2.1 Densification Limits . . . . . . . . . . . . . . . . 146

8.2.2 Exploiting Traffic Patterns in Partitioning Based Schemes . 146

8.2.3 Learning the Behaviours of Neighboring SBSs . . . . . . . . 147

8.2.4 MIMO Small Cells . . . . . . . . . . . . . . . . . . 147

8.2.5 Joint Beamforming and Scheduling . . . . . . . . . . . 148

$\begin{array}{ll}\text { List of References } & 149\end{array}$

$\begin{array}{ll}\text { Appendix A Simulation Setup } & 156\end{array}$

A.1 Network Deployment . . . . . . . . . . . . . . . . 156

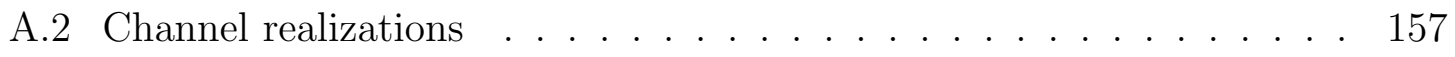




\section{List of Tables}

4.1 Simulation Parameters . . . . . . . . . . . . . . 63

5.1 Simulation parameters. . . . . . . . . . . . 85

6.1 Simulation parameters . . . . . . . . . . . . . . 114

7.1 Different scenarios for serving 2 cell edge users via 2 SBSs. . . . . . . 126

7.2 Simulation parameters . . . . . . . . . . . . . . 134

7.3 Occurrence percentage of each type of scheduling as chosen by each proposed scheme. . . . . . . . . . . . . . . . . 135 


\section{List of Figures}

1.1 A heterogeneous network. . . . . . . . . . . . . . 3

2.1 Adaptive modulation assignment [1] . . . . . . . . . . . . . 16

4.1 Utility function: a) with respect to SINR while power is fixed, and b) with respect to power while SINR is fixed. . . . . . . . . . . . . 48

4.2 SINR utility and its cost in low and high power regimes. . . . . . . . 51

4.3 Utility function w.r.t power, when the desired channel $h$ and interference $I$ are fixed. . . . . . . . . . . . . . . . . . . . . . . . . . . . . . . 52

4.4 The impact of increasing $\lambda_{n}$ beyond the RHS of (4.17) on: a) spectral efficiency, and b) energy efficiency. The number of small cells in this example is $6 . \ldots \ldots \ldots \ldots$. . . . . . . . . . . . . . . . . . 58

4.5 Relationship between the number of macrocell users and the number of their assigned subchannels using Algorithm 1. . . . . . . . . . . 63

4.6 Average aggregate spectral efficiency in the organized network topology. 66

4.7 Average border user spectral efficiency in the organized network topology. 66

4.8 Average energy efficiency in the organized network topology. . . . . . 67

4.9 Empirical CDF of the required iterations for the game-based schemes to converge (The number of SBSs is 27). . . . . . . . . . . . 67

4.10 Empirical CDF of the a) aggregate spectral efficiency, and b) border user spectral efficiency (random topology). . . . . . . . . . . . 68

4.11 Average energy efficiency in a random topology. . . . . . . . . . . . 69 
4.12 Average number of iterations to convergence (random topology). . . . 70

4.13 Average aggregate spectral efficiency in the random topology. . . . . 70

5.1 The coined distribution of the transmit power versus the distribution of the actual transmit power obtained by (5.13). . . . . . . . . 85

5.2 Spectral efficiency in different scenarios. . . . . . . . . . . . 86

5.3 Spectral efficiency comparison between the MFG-based approach and the Reuse-3 technique. TotalArea $\approx N \pi r_{F}^{2}$. . . . . . . . . . . . 87

6.1 A heterogeneous wireless network. . . . . . . . . . . . . . . 92

6.2 The relationship between price factor $\lambda_{n m}$ and $\bar{p}$ as described by the consistent condition $(C 1)$. . . . . . . . . . . . . . 100

6.3 The point that realizes consistent condition $(C 1)$. The selected operating point here is: $\bar{p}_{m}=0.3 \ldots \ldots \ldots \ldots$. . . . . . . . . 102

6.4 Cut-off channel gain $\left(h_{n m}^{(l)}\right)$ as a function of $\bar{p}_{m} \ldots \ldots \ldots$. . . . . . 108

6.5 The best utility value $\left(u^{*}\right)$ as a function of $\bar{p}_{m}$, where $h_{n m}>h_{n m}^{(l)}$. . 111

6.6 Average aggregate spectral efficiency vs. the number of small cells. . . 112

6.7 Average energy efficiency vs. the number of small cells. . . . . . . . . 112

6.8 Average border user spectral efficiency vs. the number of small cells. 113

6.9 Average area spectral efficiency vs. normalized density of small cell base stations. . . . . . . . . . . . . . . . . . . . . . . . 117

6.10 Average border user spectral efficiency vs. the number of small cell base stations. . . . . . . . . . . . . . . . . . . . . . . . 118

6.11 Average energy efficiency vs. the number of small cell base stations. . 119

6.12 Empirical CDF of the area spectral efficiency in a random deployed network (The average number of SBSs is 27). . . . . . . . . . . . 120

6.13 Empirical CDF of the border user spectral efficiency in a random deployed network (The average number of SBSs is 27) . . . . . . . . . . 121 
6.14 Empirical CDF of the required iterations for conventional game to converge (The average number of SBSs is 27). . . . . . . . . . . . . . 121

6.15 Empirical CDF of the total transmit power of MBS. . . . . . . . . . . 122

7.1 The problem of cell edge users in an open access small cells network. 125

7.2 Cumulative density function (CDF) of the sum rate (simulation parameters are show in Table 7.2) . . . . . . . . . . . . . . . . 128

7.3 (a) CDFs of the packet time delay of different proposed scheduling schemes; (b) CDFs of the packet time delay when the conventional interference channel scheduling is used. . . . . . . . . . . . . . . . . 139

7.4 CDFs of cell edge users' sum rates for different schemes. . . . . . . . 139

7.5 CDFs of the total time required to deliver 200 packets. (a) Different proposed scheduling schemes, (b) Conventional interference channel scheduling. . . . . . . . . . . . . . . . . . . . . . . . . 140

7.6 The sum rate vs. the packet arrival rate. . . . . . . . . . . . . . 140

7.7 Average packet delay. (a) Different proposed scheduling schemes, (b) Conventional interference channel scheduling. . . . . . . . . . . . . . 141

A.1 Organized HetNet: (a) low density network, (b) dense network. Green star: MBS

Green dots: MUs

Coloured triangles: SBSs

Small coloured dots: SCUs . . . . . . . . . . . . . . . . . . 157

A.2 HetNet system with random topology.

Green star: MBS

Green dots: MUs

Black triangles: SBSs

Small black dots: SCUs . . . . . . . . . . . . . . . . . . . . 158

A.3 Time-frequency fading realizations: (a) desired link, (b) interfering link.159 


\section{Nomenclature}

Here are the lists of acronyms and symbols.

\section{Acronyms:}

$\begin{array}{ll}\text { Acronym } & \text { Description } \\ \text { AMC } & \text { Adaptive Modulation and Coding } \\ \text { ASE } & \text { Area Spectral Efficiency } \\ \text { AWGN } & \text { Additive White Gaussian Noise } \\ \text { BER } & \text { Bit Error Rate } \\ \text { BF } & \text { Beamforming } \\ \text { BS } & \text { Base Station } \\ \text { CC } & \text { Consistency Condition } \\ \text { CDF } & \text { Cumulative Density Function } \\ \text { CDMA } & \text { Code Division Multiple Access } \\ \text { COMP } & \text { Coordinated Multipoint } \\ & \end{array}$




$\begin{array}{ll}\text { Acronym } & \text { Description } \\ \text { CP } & \text { Cyclic Prefix } \\ \text { dB } & \text { Decibel } \\ \text { DP } & \text { Dynamic Programming } \\ \text { EE } & \text { Energy Efficiency } \\ \text { EMA } & \text { Exponential Moving Average } \\ \text { FBE } & \text { Fair Best Effort Scheme } \\ \text { FDD } & \text { Frequency Division Duplex } \\ \text { HetNet } & \text { Heterogeneous Network } \\ \text { ICI } & \text { Intercell Interference } \\ \text { IFC } & \text { Conventional Interference Channel } \\ \text { ISI } & \text { Inter-Symbol Interference } \\ \text { IW } & \text { Iterative Water-filling } \\ \text { KKT } & \text { Karush-Kuhn-Tucker } \\ \text { LR } & \text { Lagrangian Relaxation } \\ \text { MA } & \text { Long Term Evolution } \\ \text { MA } & \text { Moving Average } \\ \text { MAcro Base Station }\end{array}$




\section{Acronym Description}

\begin{tabular}{|c|c|}
\hline MFA & Mean Field Approximation \\
\hline $\mathrm{MFG}$ & Mean Field Game \\
\hline MIMO & Multiple Input Multiple Output \\
\hline MINLP & Mixed Integer Non-Linear Programming \\
\hline MISO & Multiple Input Single Output \\
\hline $\mathrm{MU}$ & Macro User \\
\hline $\mathrm{NCO}$ & Network-Centric Objective Scheme \\
\hline $\mathrm{NE}$ & Nash Equilibrium \\
\hline $\mathrm{OA}$ & Outer Approximation \\
\hline OFDM & Orthogonal Frequency Division Multiplexing \\
\hline OFDMA & Orthogonal Frequency Division Multiple Access \\
\hline $\mathrm{PDF}$ & Probability Distribution Function \\
\hline $\mathrm{PPP}$ & Poisson Point Process \\
\hline QoS & Quality of Service \\
\hline REA & Range Expansion Association \\
\hline $\mathrm{Rx}$ & Receiver \\
\hline SA & Spectrum Avoidance \\
\hline AT & Satisfying a Quality of Service \\
\hline
\end{tabular}




\section{Acronym Description}

$\begin{array}{ll}\text { SBS } & \text { Small Base Station } \\ \text { SCU } & \text { Small Cell User } \\ \text { SE } & \text { Spectral Efficiency } \\ \text { SFR } & \text { Soft Frequency Reuse } \\ \text { SINR } & \text { Signal to Interference plus Noise Ratio } \\ \text { SNR } & \text { Signal to Noise Ratio } \\ \text { SON } & \text { Self Organizing Network } \\ \text { SS } & \text { Spectrum Sharing } \\ \text { TDMA } & \text { Time Division Multiple Access } \\ \text { TEXP } & \text { Truncated Exponential Distribution } \\ \text { Tx } & \text { Transmitter } \\ & \\ & \end{array}$




\section{Symbols:}

$\begin{array}{ll}\text { Symbol } & \text { Description } \\ D_{m m} & \text { Distance Separating MBS and MU } \\ D_{s m} & \text { Distance Separating MBS and SCU } \\ D_{s s} & \text { Distance Separating SBS and a Desired SCU } \\ D_{m s} & \text { Distance Separating SBS and MU } \\ D_{i s s} & \text { Distance Separating SBS and non desired SCU } \\ L_{m, m} & \text { Decibel (dB) Path Loss Between MBS and a MU } \\ L_{s, m} & \text { dB Path Loss Between MBS and a SCU } \\ L_{s, s} & \text { dB Path Loss Between a SBS and a Desired SCU } \\ L_{m, s} & \text { dB Path Loss Between a SBS and an Outside MU } \\ L_{i s, s} & \text { dB Path Loss Between a SBS and a SCU at another small cell } \\ f_{c} & \text { Carrier Frequency } \\ \nu_{m m} & \text { Outdoor Path Loss Exponent } \\ \nu_{m s} & \text { Indoor to Outdoor Path Loss Exponent } \\ \nu_{s s} & \text { Indoor Path Loss Exponent } \\ \alpha_{n} & \text { Loss Due to Wall Penetration } \\ \eta & \text { Fairness Weighting Factor } \\ & \end{array}$




\section{Symbol Description}

$\lambda \quad$ Price Factor

$\tau \quad$ A Time Slot Duration

$\rho \quad$ Fairness Window Size

$\Gamma \quad$ Target signal-to-interference-and-noise ratio

$\gamma \quad$ Signal-to-Interference-and-Noise Ratio

$\sigma^{2} \quad$ power of the additive white Gaussian noise

$\alpha \quad$ Fairness factor

$\theta \quad$ phase of the antenna weight

$\psi \quad$ marginal utility of SINR

$\omega \quad$ marginal utility of holding the power

$\mathcal{M}$ Set of Perfectly orthogonal OFDM Subchannels

$\mathcal{N} \quad$ Set of SBSs

$\mathcal{N}_{S C U} \quad$ Set of SCUs

$\mathcal{J} \quad$ Set of MUs

$I_{n m} \quad$ Total Interference at the $n^{\text {th }}$ Small Cell's Receiver over $m^{\text {th }}$ Subch.

$p_{j m} \quad$ Transmit Power by the $j^{\text {th }}$ Macrocell's Transmitter on $m^{\text {th }}$ Subch.

$p_{n m} \quad$ Transmit Power by the $n^{\text {th }}$ Small Cell's Transmitter on $m^{\text {th }}$ Subch.

$p_{\text {total }}^{(C)} \quad$ Total Allowable Transmit Power at the Macrocell 


\section{Symbol Description}

$p_{\text {total }}^{(F)} \quad$ Total Allowable Transmit Power at Each Small Cell

$B \quad$ Bandwidth of Each Subchannel

$R_{j} \quad$ Minimum Data Rate Required by $j^{\text {th }} \mathrm{MU}$

$\beta_{j} \quad$ A Positive Factor that Takes into Account the Required BER of $M U_{j}$

$\beta_{n} \quad$ A Positive Factor that Takes into Account the Required BER of $S C U_{n}$

$\xi \quad$ A Partition Parameter Defined by the Operator

$\mathcal{M}^{M} \quad$ Set of Macrocell Subchannels

$\mathcal{M}^{S} \quad$ Set of Small Cells Subchannels

$\mathbf{p}_{m} \quad$ Vector of all players' transmit powers over $m^{\text {th }}$ Subchannel

$u_{n m} \quad$ Utility Function in $n^{\text {th }}$ Small Cell

$u_{n m}^{*} \quad$ Best Utility Value in $n^{\text {th }}$ Small Cell

$\mathbf{H i}_{m} \quad$ Interference Channel Matrix

$\bar{h}_{-n m} \quad$ Average of the Interference Channels to the $n^{\text {th }}$ Small Cell Receiver

$\bar{p}_{-n m} \quad$ Average Transmit Power Over all Transmitter Except the $n^{\text {th }}$ Transmitter

$N_{o} \quad$ Noise Power Spectral Density

$p_{n m}^{*} \quad$ Best Response Transmit power

$\digamma\left(\gamma_{n m}\right) \quad$ A Function that Maps the SINR into Data Rate 


\section{Chapter 1}

\section{Introduction}

Since the introduction of the first cellular system, less than 30 years ago, the technology used in wireless systems has progressed spectacularly. Business arrangements, technological advancements, and focused research not only allowed ordinary people to receive voice calls, but also made it possible for them to receive anytime anywhere services, at an affordable price. In recent years, and as a result of these flourishing wireless networks, we have been confronted by an explosion of data traffic. Moreover, a 1000-fold data traffic increase is anticipated in the next decade. In other words, wireless systems are going to face exponentially increasing demands from all classes of the population. This challenge makes the powerful technological progress achieved negligible compared to what is needed to meet the current and expected demands. Designers therefore need to focus on finding methods to scale the network capacity with reasonable capital and operating expenditures.

Recent solutions such as diversity and adaptive modulation and coding schemes (AMC), which enhance spectrum utilization, are quite limited as the link capacity is very close to the theoretical limits. Furthermore, increasing the bandwidth is generally a very costly solution.

A solution guaranteed to increase the system capacity is to bring transmitters closer to their receivers. Such a solution would achieve the dual benefits of enhanced 
link budgets and increased frequency reuse. This could be implemented by deploying more infrastructures such as microcells, relays, and distributed antennas. A better and more cost efficient way is to deploy a large number of smaller base stations in each macrocell area. This can be implemented by allowing end users to deploy their own small base stations (SBSs), and hence take part in the network capital and operational expenditures. Consequently, small cell networks, whose main purpose is to maximize spectrum utilization, would play an important role in overcoming the future challenges of cellular networks.

As shown in Fig. (1.1), heterogeneous networks (HetNets) consist of a central macrocell base station ${ }^{1}(\mathrm{MBS})$ underlaid with a large number of shorter range, smaller base stations $^{2}$ (SBSs), which are wired to an IP backhaul (e.g. cable or fiber to the home). As a result, the SBSs support greater capacity in small areas, while the MBSs support large scale mobility.

\subsection{Challenges Facing HetNets}

Due to shorter radii and more spectrum reuse, small cells deployment is theoretically an economical and viable strategy for enhancing network capacity. Nevertheless, there are several challenges that can obstruct a successful HetNets deployment. They are listed as follows:

- As cells are packed in a relatively small area, inter-cell interference (ICI) would be the main capacity limiting factor of heterogeneous networks [2]. In HetNets, there are two sources of inter-cell interference. The first interference arises when a macro base station coverage area overlaps with many small BSs' coverage areas, causing cross layer interference [3]. The second interference emerges

\footnotetext{
${ }^{1}$ Macro BSs cover an area with a radius of more than $500 \mathrm{~m}$.

${ }^{2}$ Small BSs are base stations that can cover femto and pico cells whose areas' radii are less than $30 \mathrm{~m}$ and $300 \mathrm{~m}$ respectively.
} 


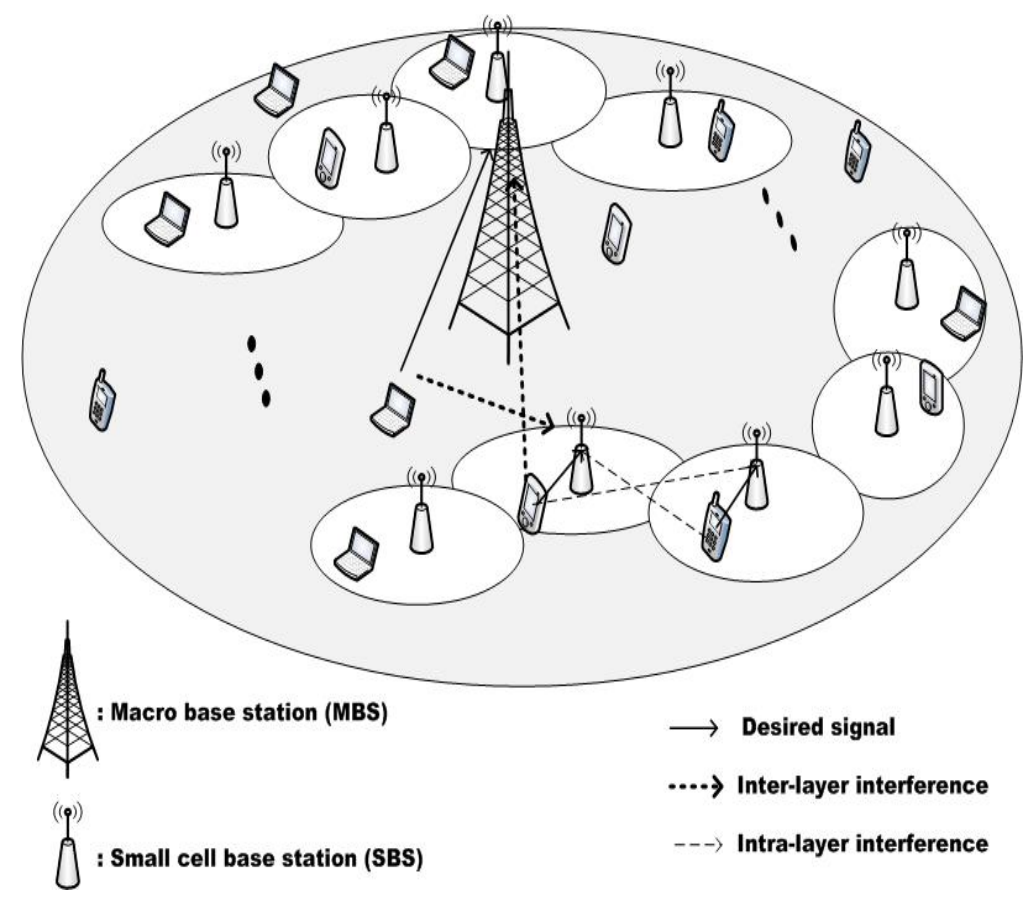

Figure 1: A heterogeneous network.

when the small BSs' coverage areas are themselves partially overlapping with each other, causing co-layer interference ${ }^{3}[3]$. Because of the lack of coordination between different BSs, a distributed interference mitigation scheme is indeed crucial for future HetNet systems.

- It is expected that most small base stations would be deployed in random locations. This unplanned deployment is uncorrelated with the network traffic pattern and resource requirements. Therefore, new self-organizing procedures are required for reconfiguring, re-optimizing and self-healing.

- The current mobility management and handover mechanisms may not be sufficient for HetNets, since each mobile would experience several handover events in a relatively short distance and/or time. The less complex and less expensive

\footnotetext{
${ }^{3}$ The inter-cell interference is the mutual interference between nodes located in different cells while the intra-cell interference is the mutual interference between nodes located in the same cell. Therefore, cross-layer interference and co-layer interference both fall in the category of inter-cell interference.
} 
SBS is not capable of rapidly tracking the handover lists as users come and go. Thus, developing a low rate handover algorithm is required for stable HetNets.

- SBSs would be owned by the end users who will probably not allow public access to them, unless they have been tempted by some incentives. Therefore, closed access small cells would create and receive uncoordinated interference with a faster timescale variation.

- If incentives were arranged for SBSs owners to open their SBSs for roamers, security and privacy would be important issues for open access HetNets. In terms of resource allocation, the priority of the SBS owner over the macrocell roamers has to be arranged. Furthermore, for fair billing purposes, operators need to differentiate between the traffic of SBSs owners and that of the roamers. Tradeoffs between open access and closed access HetNets were provided in [4].

- Synchronization is another challenge to the success of HetNet systems. Synchronization is required to align received signals in order to minimize co-channel interference. Moreover, central handover coordination between the macrocell and the small cells is difficult to design in HetNets. Therefore, synchronization and timing play a crucial role in the success of a handover. The problem is that SBSs are connected to an IP backhaul, which usually suffers from packet jitter, which may create errors in synchronization.

- Since the main objective of small cells deployment is maximizing network capacity via spectrum reuse, a user may associate with a BS whose received power is weaker than another BS's, as long as the gain (in rate) due to spatial reuse is greater than the loss (in rate), due to higher interference. Therefore, a new cell association mechanism, which takes into account the spectrum reuse factor, should be created. 


\subsection{Problem Statement}

Since the main capacity limiting factor of OFDM-based heterogeneous networks is the inter-cell interference [2], this research is dedicated to addressing this challenge. The interference management can be achieved by cleverly allocating the resources over

the cells. In a heterogeneous network (as shown in Fig.1.1), there are two sources of inter-cell interference: the mutual interference between a macrocell and each small cell, and the mutual interference among small cells. The former source of interference, which is due to an overlap between the area of a macrocell and those of many small cells, is called cross-layer interference [3]. On the other hand, the latter source is called co-layer interference, which arises when SBSs' coverage areas are themselves partially overlapping each other [3]. In such conditions, each BS should be capable of deciding its optimal transmit power on each radio resource, at every given time, with the objective of maximizing the satisfaction level of its users. The optimal transmit power is a power level that balances between the need to maximize the received power at a desired destination and the need to minimize the negative reaction of other transmitters, which manifests as interference experienced by that destination. A network whose BSs are capable of taking such optimal decisions is called a self organizing network (SON). The objective of this research is to equip each BS with such capability. In particular, we consider an OFDM-based heterogeneous network consisting of several SBSs located within a macrocell area that is simultaneously served by one MBS.

The OFDM-based network is chosen because most standardization bodies still consider OFDM to be the best transmission scheme for the future networks (e.g. 5G and beyond). The most appealing feature of the OFDM transmission scheme is its ability to suppress the frequency selective fading. Moreover, OFDM-based networks are free of intracell interference, assuming perfectly orthogonal subchannels, whereas 
the most limiting factor of such networks is inter-cell interference.

\subsection{Main Contributions and Thesis Organization}

In this thesis, we aim to propose holistic and fully distributed resource allocation and interference management for HetNet systems. The contributions are as follow:

1. In HetNet systems with few to a moderate number of small cells:

- A dynamic partitioning of the resources between the macrocell and small cells is proposed by formulating an optimization problem. This proposed partitioning is flexible, and hence the operator is able to adjust/implement the required operational strategies to achieve certain goals and satisfy different interests.

- In addition to eliminating cross-layer interference, an energy saving policy is incorporated into the problem formulation in order to increase the energy efficiency of the MUs.

- An iterative algorithm is designed to solve the above MINLP optimization problem. The conditions needed to directly examine the MUs' satisfactions are identified.

- To control the co-layer interference, a non-cooperative game is formulated, and two elements are analyzed: the utility function, whose penalty part is quadratic in the transmit power, as well as the equilibrium existence and uniqueness. The outcome of the game is then identified. Furthermore, a distributed iterative algorithm, which quickly converges to the equilibrium point in a real-time operation, is suggested. To the best of our knowledge, There is no literature that examine and analyze a game with a utility function whose penalty part is quadratic with respect to the transmit power. 
- Two forms/versions of the game, which differ in their action sets, are proposed and experimentally compared.

2. To the best of our knowledge, we have not found a literature that considers applying mean field game theory into dense HetNets. Therefore, in HetNet systems with a large number of small cells (i.e., densified network):

- We formulated the inter-cell interference management issue as two nested problems; an overlay problem at the MBS level and an underlay problem at the SBS level. In the overlay problem, the MBS selects the optimal action first, which satisfies its associated MUs with a minimum amount of cross-layer interference, and also considers the reaction of the SBSs.

- The underlay problem is then formulated as a non-cooperative game among the SBSs. The mean-field theory is exploited to help decouple a complex large-scale optimization problem into a family of localized optimization problems. Thus, each SBS can implement its policy by using only its local information and some macroscopic information. Furthermore, by using the same technique, the overlay problem at the MBS level is converted into a single cell resource allocation problem.

- In addition to the achieved tradeoff between spectral efficiency and energy efficiency, our approach can substantially reduce the communication overhead of interference management and resource allocation in HetNets.

3. In an open access small cells network, the throughput of the cell edge users, who are the real victims of the inter-cell interference, is enhanced significantly. In this case, the proximity of the small BSs is viewed as an advantage to be exploited via smart and optimal scheduling. Different schemes are proposed to enhance the cell edge users rate to at least double the existing rate, and 
substantially reduce the average packet delay. In these schemes, the SBSs decide their transmission types by solving a common problem that does not need any inter-SBS information exchange. Moreover, to ensure a better performance in terms of packet delays, upper layer information is also taken into account.

\subsubsection{Thesis Outline and List of Publications}

This thesis can be divided into two main parts. The first three chapters present the introduction, the background and the literature review. Then, the next four chapters are my contributions, while the final chapter concludes the work. The breakdown of the thesis and the produced papers and reports cross-referenced to individual chapters are as follows:

- For a greater comprehension of the subsequent chapters, a detailed background as well as essential preliminary knowledge are introduced in Chapter 2.

- Chapter 3 serves as a literature review and a panorama of the state of the art.

- Chapter 4 presents a fairness-aware distributed interference management scheme using a non-cooperative game theory designed for heterogeneous networks with a moderate number of small cells. The following journal paper and conference paper are the outcome of that chapter:

- Ali Y. Al Zahrani and F. Richard Yu, "An Energy-Efficient Resource Allocation and Interference Management Scheme in Green Heterogeneous Networks Using Game Theory", submitted to IEEE Transaction on Vehicular Technology, April 2014, revised in Sep. 2014.

- Ali Y. Al Zahrani and F. Richard Yu, "A Game Theory Approach for Inter-Cell Interference Management in OFDM Networks", in Proc. IEEE ICC'11, pp. 1-5, Kyoto, Japan, June 2011. 
- In Chapter 5, a mean-field game based scheme is introduced to distributively tackle the inter-cell interference in HetNet systems with large number of small cells. The following technical report and paper are the outcome of that chapter.

- Ali Y. Al-Zahrani, F. Richard Yu and Minyi Huang, "Uplink Power Control in HetNet via Mean Field Games", Deliverable D3/2012, submitted to Huawei Canada, 28 June. 2012.

- Ali Y. Al Zahrani, F. Richard Yu and Minyi Huang, "A Mean-Field Game Approach for Distributed Interference and Resource Management in Heterogeneous Cellular Networks", in Proc. IEEE GLOBECOM'13, pp. 49644969, Atlanta, GA, Dec. 2013.

- A novel decentralized and full spectrum sharing-based interference control mechanism in dense heterogeneous networks is introduced in Chapter 6. The following technical report, journal paper and conference paper are the outcome of the content of Chapter 6:

- Ali Y. Al-Zahrani, F. Richard Yu and Minyi Huang, "A Mean-Field Game Theoretic Approach For Fair and Energy-Efficient Distributed Interference Control in Heterogeneous Cellular Networks", Deliverable D2/2013, submitted to Huawei Canada, 29 Aug. 2013.

- Ali Y. Al Zahrani, F. Richard Yu and Minyi Huang, "A Joint Cross-Layer and Co-Layer Interference Management Scheme in Dense Heterogeneous Networks Using Mean-Field Game Theory", Accepted in IEEE Transaction on Vehicular Technology, Oct. 2014.

- Ali Y. Al Zahrani, F. Richard Yu and Minyi Huang, "A Distributed Interference Control Scheme in Large Cellular Networks Using Mean-Field Game Theory", in Proc. IEEE PIMRC'13, pp. 3339-3343, London, UK, 
Sep. 2013.

- Chapter 7 presents a novel scheme that enhances the throughput of cell edge users in small cells via optimal scheduling using binary linear optimizations. The following paper is the outcome of Chapter 7 .

- Ali Y. Al Zahrani and F. Richard Yu, "Enhancing Cell Edge Users Performance in Open Access Small Cells Networks: A Cross Layer Approach", Accepted and to be presented in IEEE GLOBECOM14, Austin, TX, Dec. 2014.

- Chapter 8 summarizes the thesis conclusions with a discussion of the possible extensions and relevant research directions. 


\section{Chapter 2}

\section{Preliminaries and Background}

This chapter reviews the basic characteristics of wireless communication systems and provides a deep overview of some gadgets used in this research.

\subsection{Channel Model}

Channel models often split the effects of propagation impairments into path loss due to distance, shadow fading or shadowing, and multi-path fading. In the following section, we explain the channel model selected for this thesis in greater detail.

Path loss (or attenuation) is the decline in power density of an electromagnetic wave as it propagates through space. Such a reduction can be due to free-space loss, diffraction, reflection, and/or scattering. In free space, path loss is generally assumed to be deterministic at a given transmit-receive distance. In addition, path loss is influenced by the environment (urban or rural), by the propagation medium (dry or moist air), by the transmission frequency, and by the height of the antennas. The path loss models adopted throughout this thesis are based on the International Mobile Telecommunications (IMT) standard [5]. In the following, we describe these models, which capture the essence of signal propagation for different links:

- The decibel $(\mathrm{dB})$ path loss between MBS and a macro user (MU) is modelled 
as:

$$
L_{m, m}=30 \log _{10}\left(f_{c}\right)-71+10 \nu_{m, m} \log _{10}\left(D_{m, m}\right)
$$

where $f_{c}$ is the carrier frequency, $\nu_{m m}$ is the outdoor path loss exponent, and $D_{m m}$ is the distance separating MBS and MU.

- The dB path loss model between MBS and a small cell user (SCU) is given by:

$$
L_{s, m}=30 \log _{10}\left(f_{c}\right)-71+W+10 \nu_{m, m} \log _{10}\left(D_{s, m}\right)
$$

where $W$ is the loss due to wall penetration during outdoor to indoor propagation.

- The dB path loss model between SBS and its associated small user (SCU) is given by:

$$
L_{s, s}=37+10 \nu_{s, s} \log _{10}\left(D_{s, s}\right),
$$

where $\nu_{s s}$ is the indoor path loss exponent.

- The path loss model between SBS and the outside macro user (MU) is:

$$
L_{m, s}=37+W+10 \nu_{m, s} \log _{10}\left(D_{m, s}\right)
$$

- Finally, the dB path loss model of the interference between a SBS and a SCU at another small cell is provided by:

$$
L_{i s, s}=37+2 W+10 \nu_{m, s} \log _{10}\left(D_{i s, s}\right),
$$

where $2 W$ is due to a double wall penetration during indoor to indoor propagation. Note that the path exponents from indoor to outdoor and indoor to indoor are almost the same. 
These models are extended from the COST231 empirical model [6] for IMT standard $[5]$.

In addition to path loss due to distance, a transmitted signal will be attenuated by objects blocking the path between the transmitter and the receiver. This attenuation is referred to as shadow fading or shadowing. In this thesis, it is modelled as a Gaussian distribution in dB scale (log-normal distribution in linear scale) [6,7].

Multi-path fading describes the received power variations and rapid fluctuations of the amplitudes and phases due to the constructive and destructive addition of multipath signal components. Such variations and fluctuations occur over a much shorter period of time and/or distance compared to path loss and shadowing. Generally, multi-path fading is influenced by spatial (physical) channels, the speed of the mobile user, the speed of the surrounding objects, and the bandwidth of the transmitted signal [7]. When the channel consists of several weak paths ${ }^{1}$, that is

$$
h(t)=\sum_{i=1}^{n(t)} a_{i}(t) \delta\left(t-\tau_{i}(t)\right)
$$

where $n(t)$ is the number of paths at time $t, a_{i}(t)$ is the gain of path $i$ at time $t$, and $\tau_{i}(t)$ is the time delay associated with path $i$ at time $t$. It is common for the Rayleigh distribution to be considered a potential model to describe the statistical variations of the received envelope of a fading signal $[6,7]$. The Rayleigh distribution probability density function (pdf) is:

$$
f_{R}(r)= \begin{cases}\frac{r}{\beta^{2}} \cdot e^{-\frac{r^{2}}{2 \beta^{2}}} & (0 \leq r \leq \infty) \\ 0 & (r<0)\end{cases}
$$

where $\beta^{2}$ is the time-average power of the received signal.

\footnotetext{
${ }^{1}$ Which is the case of the interfering channels and of the many desired channels
} 
Since this research is concerned with inter-cell interference, where most of the signal components are weak, a multi-path model based on the Rayleigh distribution is adopted in this research. In this case, the channel power gain would vary according to an exponential distribution, due to the fact that the square of a Rayleigh random variable is an exponential random variable. Moreover, since the physical layer transmission scheme in the considered HetNets is OFDM, wireless channel may experience flat and slow fading (since users within houses are relatively static).

In regards to the interference effect, the focus of this thesis is only on the inter-cell interference in OFDM HetNet systems. Thus, the SINR can generally be expressed as follows:

$$
\gamma=\frac{h_{i, i} p_{i}}{I_{i}+\sigma^{2}}
$$

where $h_{i, i}$ and $p_{i}$ are respectively the channel gain of the desired signal and the transmitted power. $I_{i}$ is the overall interference experienced by link $i$, whereas $\sigma^{2}$ is the power of the additive white Gaussian noise (AWGN). While path loss, shadowing, and multi-path are degrading the SINR by degrading the power of the desired channel $h_{i, i}$, interference is reducing the SINR by increasing the value of the denominator. If user $i$ is a macro user, then $I_{i}$ will be the overall interference arriving from all small cells. On the other hand, if user $i$ is a small cell user, $I_{i}$ will consist of the interference arriving from the macro cell plus the interference from all remaining small cells.

While the interference affects the SINR in the same way as the noise does, the interference has a greater adverse impact on the SINR, due to the higher power it possesses as well as the severe randomness of its nature. Whereas the noise can be modelled by the Gaussian distribution, interference is very hard to analytically model since there are many random variables involved; there is only one case where the interference can be efficiently modelled. When there are a large number of identical interferers, interference can then be regarded as a Gaussian random variable owing 
to the central limit theorem.

Remark: In this thesis, it is assumed that the system is reasonably immune to the inter-macrocell interference due to interference coordination such as static or semi-static fractional frequency reuse.

\subsection{Channel Adaptation}

Due to the large fluctuations in a wireless channel, we can often gain from adapting transmissions to the channel variations (called link adaptations). Parameters that can be adapted are, for instance, the modulation constellation size and the coding rate. Transmission techniques that do not have the ability to adapt to the fading conditions require a modulation and coding scheme that guarantees an acceptable bit error rate in instances when channel quality is poor. These systems are actually designed for worst-case conditions, where the signal loss might reach up to $30 \mathrm{~dB}$ [6]. Designing for worst-case conditions leads to a very inefficient utilization of resources. Therefore, channel adaptation can increase throughput (and conversely decrease the required transmitting power or reduce the bit error rate (BER)) by exploiting the favourable channel instances. Figure (2.1) shows how the choice of modulation occurs. Lets assume, for example, that the required BER is $10^{-3}$, then the transmitter should be in idle mode, in case the SINR is less than $7 \mathrm{~dB}$. However, if the SINR is higher than $7 \mathrm{~dB}$ and lower than $11 \mathrm{~dB}$, the transmitter should transmit using BPSK mode. The transmitter should transmit with 4-QAM if the SINR falls between $11 \mathrm{~dB}$ and $17 \mathrm{~dB}$, and so on and so forth.

Adaptive modulation systems require that some information about the state of the channel be provided to the transmitter. In time division duplex systems, channel reciprocity can be exploited by assuming that the channel remains fixed for the duration of uplinks and downlinks. Alternatively, the channel knowledge can also be 


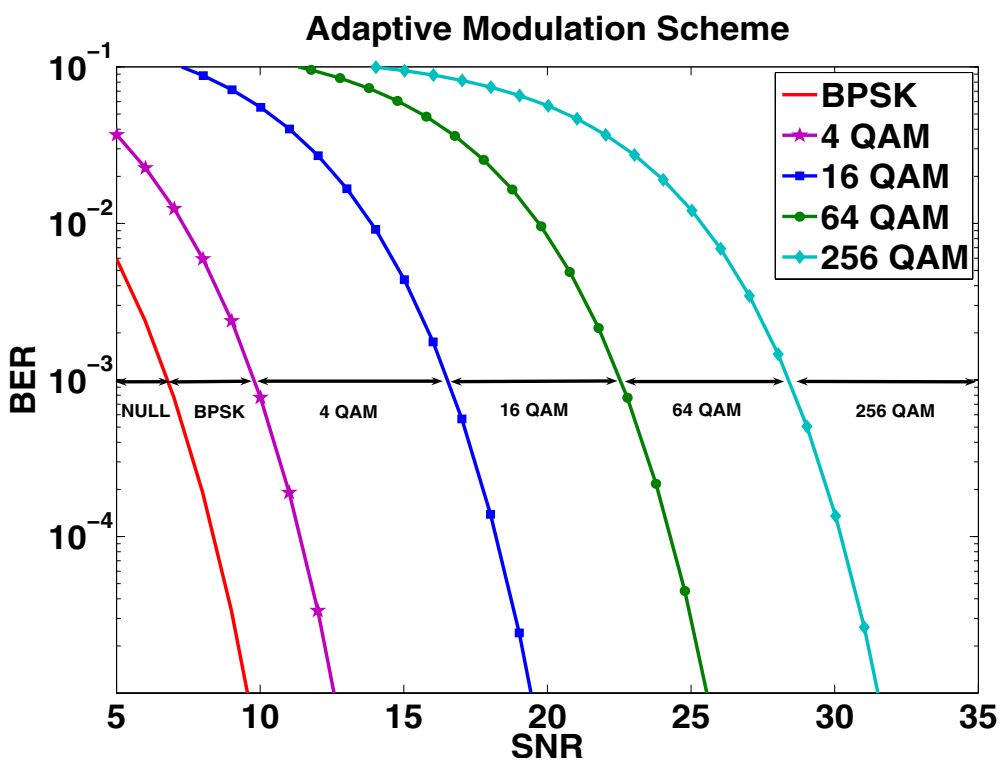

Figure 2.1: Adaptive modulation assignment [1].

directly measured at the receiver, and then fed back to the transmitter.

Although the adaptive modulation and coding scheme switches from one modulation level to another in a discrete manner, with respect to SINR, the following spectral efficiency formula is used to analytically capture the effect of the link adaptation scheme, in a continuous fashion, with SINR [8] [9]:

$$
\eta=\log _{2}\left(1+\frac{-1.5 \gamma}{\ln (5 B E R)}\right)
$$

where $\eta$ is in bps/Hz. Throughout this thesis, it is assumed that all wireless nodes are able to perform a link adaptation technique.

\subsection{Mathematical Tools}

In this section, we briefly explain various mathematical gadgets and helpful tools that are employed in our research. 


\subsubsection{Game Theory}

In chapter 4 , game theory results were exploited to control the co-layer interference. Game theory is a mathematical discipline whose goal is to model, describe, and analyze a competitive situation in which two or more decision makers have to select specific actions that may cause mutual conflict. In recent years, game theory has been applied to networking and wireless communication to solve competitive situations, mostly in routing, and resource allocation problems. For instance, transmitting entities in a wireless communication system are considered rational decision makers that control their communication parameters to cope with the scarcity of radio resources. Accordingly, they attempt to resolve such conflicts by adopting certain actions such as transmitting later, changing their transmission channel, or adjusting their transmission power.

A game is characterized by a set of rational players, a set of actions associated with each player, and a utility function for each player: $\mathcal{G}=\left(\mathcal{K},\left\{\mathcal{A}_{k}\right\}_{k=1}^{K},\left\{u_{k}\right\}_{k=1}^{K}\right)$, where

- $\mathcal{K}=\{1,2, \ldots K\}$ is the set of rational players.

- $\mathcal{A}_{k}=\left\{a_{k 1}, a_{k 2}, \ldots, a_{k v_{k}}\right\}$ is the set of actions available to player $k$, where $v_{k}$ is the number of actions available to player $k$.

$\mathcal{A P}=\mathcal{A}_{1} \times \mathcal{A}_{2} \times \ldots \times \mathcal{A}_{K}=\left\{\left(a_{1}, a_{2}, \ldots, a_{K}\right)\right\}$ is the set of action profiles, which means all the possible combinations of actions for the players, where $a_{k} \in \mathcal{A}_{k}$.

- $u_{k}: \mathcal{A}_{1} \times \mathcal{A}_{2} \times \ldots \times \mathcal{A}_{K} \longrightarrow \Re$ is the utility function for each player $k$. It maps an action profile to a numerical real number. If $a_{k 1}, a_{k 2} \in \mathcal{A}_{k}$, player $k$ prefers $a_{k 1}$ over $a_{k 2}$ if and only if $u_{k}\left(a_{k 1}\right) \geq u_{k}\left(a_{k 2}\right)$.

Note that player's utility values only convey ordinal information. In other words, if $u_{k}(a)>u_{k}(b)$, it means that player $k$ prefers action $a$ over action $b$. As a result, 
utility functions can take negative values [10].

In a non-cooperative game, each player selects the action which maximizes its own utility, i.e.

$$
a_{k}^{*}=\arg \underset{a_{k} \in \mathcal{A}_{k}}{\operatorname{maximize}} u_{k}, \forall k=1, \ldots, K
$$

Therefore, Nash equilibrium (NE) is an action profile $\left(a_{1}^{*}, a_{2}^{*}, \ldots, a_{K}^{*}\right)$ in which no player can unilaterally enhance its own utility. Mathematically:

$$
u_{k}\left(a_{k}^{*}, a_{-k}^{*}\right) \geq u_{k}\left(a_{k}, a_{-k}^{*}\right) \quad \forall a_{k} \in \mathcal{A}_{k} \text { and } k \in \mathcal{K} .
$$

where $a_{-k}^{*}=\left(a_{1}^{*}, \ldots, a_{k-1}^{*}, a_{k+1}^{*}, \ldots, a_{K}^{*}\right)$. At NE, the players would not benefit from changing their own strategy; thus, NE is a stable outcome of the game $\mathcal{G}$.

Nash equilibrium can be obtained by using the method of best response of the players. Consider a player $k$. For any given action from its competitors (i.e. $a_{-k}$ ), the best response $B_{k}$ for player $k$ is the action $a_{k} \in \mathcal{A}_{k}$ that maximizes its utility, i.e.,

$$
B_{k}\left(a_{-k}\right)=\left\{a_{k} \in \mathcal{A}_{k}: u_{k}\left(a_{k}, a_{-k}\right) \geq u_{k}\left(a_{k}^{\prime}, a_{-k}\right) \forall a_{k}^{\prime} \in \mathcal{A}_{k}\right\} .
$$

As a result, the Nash equilibrium can be found by identifying the best response of each player given other players' actions. After considering all possible combinations of the players' actions, the Nash equilibrium is identified as the set of the best responses of all the players. Consequently, the game solution (i.e. the equilibrium of the game) is the action profile adopted by the players such that none of the players want to deviate from it. Formally, at a Nash equilibrium $\left(a_{1}^{*}, a_{2}^{*}, \ldots, a_{K}^{*}\right)$,

$$
a_{k}^{*} \in B_{k}\left(a_{-k}\right), \quad \forall k \in \mathcal{K} .
$$


In brief, game theory has two important characteristics: individualism and interdependence. Individualism influences rationality and self-interest, while interdependence determines the action of each player in response to its competitors' actions (i.e. a player's utility does not only depend on one's actions, but it also depends on the other players' actions).

Wireless user equipments (UEs) that are located in adjacent cells and operate on the same subchannel, are indeed affecting each other through inter-cell interference. This interaction can be modelled as a game whose players are those UEs that are competing for network resources (i.e. bandwidth and energy). Obviously, any action taken by a user affects the performance of the other users. Accordingly, game theory is a natural method of studying such interactions and resolving such conflicts.

\subsubsection{Mean-Field Game Theory}

Mean-field game theory (MFG) and mean-field approximation concepts are at the heart of the second and third research in chapters 5 and 6 . Therefore, this subsection, as well as the next, serve as preliminaries to that research. MFG is an emerging field that investigates the interaction between a large number of players who take conflicting decisions within a stochastic setting. In the traditional game theory, when the number of players exceeds the order of tens, the problem of dimensionality appears, and suddenly the analysis and the tracking of the game become tedious, and the equilibrium computation becomes infeasible [11-14]. On the other hand, MFG theory makes use of the existence of a large number of players to average out their effect on the game and hence comes up with brief information. In other words, MFG exploits the "law of large numbers" to aggregate the effect of the other players. It works as follows: consider a regime in which the number of players grows large. In such a regime, the aggregate effect of opposing players seen by a single player is similar to "noise". Thus, solving a player's decision problem can lead to finding its 
optimal strategy. Since each player is small relative to the rest of the system, the law of large numbers suggests a natural consistency condition:

- each player should have selected an optimal action given the expected behaviour effect in the system; and

- the aggregate behaviour effect should result from the optimal actions of the players.

Interestingly enough, the growth of population is viewed by the traditional game theory as an impediment to analysis, while it is treated by MFG as an opportunity for simplifying the analysis of the equilibrium. The underlying reason is that systems have certain properties that emerge only if these systems are sufficiently large and complex [15]. Pertaining to the accuracy, the performance of MFG closely approaches the performance of conventional game theory as the number of players increases largely $[13,16]$. There are three fields that have contributed effectively to the development of the MFG theory:

- Physics:

In physics, describing the dynamics or equilibrium of a system of many particles by modelling all the inter-particles interactions is really prohibitive due to the enormous number of interactions. Luckily, mean-field game theory provides an effective methodology for handling a wide variety of these situations. Basically, it is possible to create an excellent approximation by introducing one or two intermediate variables (e.g. system pressure or temperature) that brief all interparticle interactions. In summary, the main contributions of this field are the concepts introduced by the mean-field theory.

- Economic Equilibrium:

In this field, agents in the market have little concern about each other. Indeed, 
each one is only concerned with his own interests and looks only to market prices. In other words, in the theory of economic equilibrium, prices are the intermediate variables that brief the social interactions. These interactions between agents are the main interests of economists. For a long time, their goal was to determine how rational behaviour sets the market prices.

- Game Theory:

This is the field that has had the greatest influence on MFG. Its impact is due to extensive studies involving the limit of a large class of N-player games, assuming $\mathrm{N}$ goes to infinity. The results show that inter-individual complex actions/strategies (selected by taking into account every specific player) cannot be implemented by the players, because from their perspective every other player is increasingly lost in the crowd. Moreover, the effect of one individual player on the game is infinitesimally small, and if we interchange any two players, the output of the game would not change. In other words, the identity of the players is irrelevant and nothing is dependent on the individual.

Therefore, mean-field games model situations of competition between a large number of rational players/agents that play non-cooperative games under certain symmetrical assumptions (i.e. the available actions/strategies for all the players are the same). From the above discussion, we derive the following properties for mean-field games:

- Each player is infinitesimal in a mass of other players. Consequently, the contribution of each player on the MFG is infinitesimally small.

- Each player selects its action/strategy according to its own interests, condition/state, and the condition/state of the infinite mass of other players (i.e. mean-field value) who simultaneously select theirs the same way. 
- Accordingly, when analyzing the game and finding the equilibrium, the study of one typical (representative) player is sufficient.

- As the number of players tends toward infinity, the equilibrium of MFG approaches the Nash equilibrium (NE) in the case of a static game, or the Markov perfect equilibrium (MPE) in the case of a repeated dynamic game.

- When approximating a N-player game by MFG, a special technique of approximation (usually mean-field approximation borrowed from statistical physics) is employed to allow for the restriction of the actions/strategies of the players, since the actions in MFG don't directly depend on the actions of individual players .

In hyper-dense HetNets, where small cells are largely packed in every unit area, the traditional game theory solutions would be computationally intractable. Furthermore, any distributed iterative algorithm would take longer to converge. In MFG, however, each transmitter optimizes its actions given only the long-run average statistics of the other devices, rather than the whole instantaneous vector of its competitors actions [17]. Hence, a transmitter reacts to much simpler aggregate statistics than the behaviour of the individual devices. Moreover, the computation of the equilibrium and the algorithm convergence are much simpler and faster than those in the conventional game theory.

\subsubsection{Mean Field Approximation (MFA)}

Mean field approximation ${ }^{2}$ is a well established theory in statistical physics. It essentially aims to approximate the system behaviour of interacting spins in thermal equilibrium. MFA simplifies the system by replacing the complexity of interactions between spins by averaged effective fields acting on individual spins. In other words,

\footnotetext{
${ }^{2} \mathrm{MFA}$ is sometimes called the self-consistent field theory.
} 
MFA approximates a coupled system in equilibrium with the model of a non-coupled system in which each spin is under the influence of an external field whose value is the average of all the forces exerted by the other spins. In the mean-field approximation model, spin variables are therefore independently distributed. Moreover, under the assumptions of homogeneity ${ }^{3}$ and regularity ${ }^{4}$, they are also identically distributed [18]. Thus, the behaviour of the system is reduced to a single spin problem; however, this approximation has to be done under certain consistent conditions that all spins must adhere to. More importantly, the average drawn from the resultant decoupled system has to equal the originally assumed average of the effective field. This constraining condition would cleverly maintain/model the actual correlation among spins.

In our problem, All small cells share the same properties (size, functions) in addition to the identical game properties (action set and utility function). Therefore, the homogeneity assumption is satisfied. Regarding the regularity assumption, the designed utility function, which is identical among all small cells, is chosen to be smooth function. Accordingly, we borrow this concept and apply it in a small cells network whose SBSs interact with each other, making the system very coupled. Hence, MFA can help simplify the analysis and approximates the equilibrium behaviour of a system with too many small cells.

\subsubsection{Hungarian Algorithm}

Suppose we have $n$ resources that we want to assign to $n$ tasks on a one-to-one basis. Suppose also that we know the cost of assigning a given resource to a given task. We wish to find an optimal assignment that minimizes the total cost.

Let $c_{i, j}$ be the cost of assigning the $i^{\text {th }}$ resource to the $j^{\text {th }}$ task. The cost matrix

\footnotetext{
${ }^{3}$ Homogeneity is a statistical term which means that the properties of any part of the data set are same as any other part.

${ }^{4}$ Regularity is the notion of smoothness.
} 
can be defined as the $n \times n$ matrix

$$
\begin{aligned}
& \text { Task }_{1} \text { Task }_{2} \quad \cdots \quad \text { Task }_{n} \\
& \begin{array}{l}
\text { Resource }_{1} \\
\text { Resource }_{2} \\
\vdots \\
\text { Resource }_{n}
\end{array}\left(\begin{array}{cccc}
c_{1,1} & c_{1,2} & \cdots & c_{1, n} \\
c_{2,1} & c_{2,2} & \cdots & c_{2, n} \\
\vdots & \vdots & \ddots & \vdots \\
c_{n, 1} & c_{n, 2} & \cdots & c_{n, n}
\end{array}\right) .
\end{aligned}
$$

To properly define the Hungarian algorithm and clearly explain its procedure, certain terms shall first be defined.

Definition 1. An assignment is a set of $n$ entry positions in the cost matrix, no two of which lie in the same row or column.

Definition 2. The cost of a given assignment is the sum of the $n$ entries of that assignment.

Definition 3. The optimal assignment is the assignment with the smallest possible cost.

Now, the Hungarian algorithm (also called the Kuhn-Munkres algorithm) can be defined as follows:

Definition 4. The Hungarian algorithm is a useful method that finds an optimal assignment for a given cost matrix in polynomial time.

The procedure of the Hungarian algorithm is summarized as follows:

Step 1. For each row, subtract the minimum entry from all the entries.

Step 2. For each column, subtract the minimum entry from all the entries.

Step 3. Draw the minimum number of vertical and horizontal lines such that all zero entries in the cost matrix are covered. 
Step 4. Test for optimality:

- If the minimum number of covering lines is $n$, an optimal assignment of zero entries is possible and the algorithm is finished.

- If the minimum number of covering lines is less than $n$, an optimal assignment of zero entries is not yet possible. In such a case, proceed to Step 5.

Step 5. Determine the smallest entry that is not covered by any line. Subtract this entry from each uncovered row, and then add it to each covered column. Return to Step 3. 


\section{Chapter 3}

\section{Resource Allocation and Interference Management in HetNets: A Literature Review}

In this chapter, we review the main contributions and landmark papers that have proposed significant resource allocation solutions to combat inter-cell interference in OFDM-based heterogeneous networks. In traditional cellular networks, intercell interference control techniques can be classified as signal processing based techniques and interference avoidance based techniques. Examples of the former category are interference cancellation, interference randomization, and beamforming techniques [19-21]. For the latter, the system applies certain restrictions on the available resources, in a coordinated manner, between cells [21]. This process is also known as frequency reuse, and the restrictions applied can be in terms of frequency, time, and/or power domain. A frequency reuse scheme can be of the "hard frequency reuse" or the "fractional frequency reuse" type, with an integer or a rational reuse factor, respectively [22]. Furthermore, the reuse factor can be static, semi-static, or fully dynamic, depending on how the restriction pattern changes over time. Since the interference structure in HetNets is different than in conventional cellular networks, the 
problem of inter-cell interference in HetNets has recently attracted a lot of research attention.

\subsection{General Resource Allocation and Interference Management Schemes}

Distributed joint association and resource allocation algorithms have been designed in [23] with the objective of maximizing the sum utility of average rates. In HetNets, the maximum transmit power of a BS varies depending on its size (i.e. femto, pico, or micro), whereas the maximum power of each user equipment (UE) is identical. Thus, if the cell association is based on the received power, the downlink coverage area for different BSs will vary due to the different transmit power budgets. On the other hand, the uplink coverage areas of different BSs will roughly be alike and depend only on the channel power gains. This asymmetrical nature between downlink and uplink coverage areas motivates the range expansion-based association (REA) mechanism proposed in [23]. The REA solely depends on the channel power gains. Since this type of cell association may suffer higher downlink interference, the authors argued that a user may be associated with a BS whose received power is weaker than another BS's, provided that the loss (in rate) due to the higher interference is outweighed by the gain (in rate) due to spatial reuse. Moreover, they showed that some resource partitioning among adjacent cells could alleviate a great deal of interference. The authors experimentally demonstrated that the REA is slightly better than the traditional association mechanism, but that when the resources are partitioned (even with simple fixed partitioning), the REA leads to substantial throughput gains compared to the traditional association.

To improve interference management, the authors in [23] further formulated a 
semi-static resource partitioning problem with the objective of maximizing the sum utility of average rates. However, they only provided a qualitative solution. Furthermore, they described how the traffic in smaller base stations is more dynamic than that in MBS, leading to increased temporal interference variations from the underlay small cells. In particular, since there are a few users in every small cell, there may be time slots where there are no packets waiting for transmission. To exploit the spatial-temporal traffic variations, a fast dynamic interference management method was suggested, where the semi-static allocated resources of a free-queue cell could be used by adjacent BSs in a faster time-scale.

A distributed joint resource allocation scheme for minimal energy consumption has been proposed in [24]. According to the received CQI from every user, each BS independently solves an optimization problem to update bit load, resource block (RB) allocation and power allocation. It is assumed that BSs carry out their optimization at different instants in time. The authors formulated linear integer programming problem whose running time is quite lengthy. Therefore, they resorted to a two-level optimization method to achieve near-optimal performance with a notable running time improvement. Their method incurs slight increase in the cost function (about $\% 6.37)$.

To improve the fairness among the cells, an iterative cooperative power allocation algorithm has been designed in [25]. Initially, the authors formulated an NP hard optimization problem. Then, they resorted to a suboptimal algorithm in which each cell performs power allocation based on enhanced modified iterative water filling algorithm to minimize the interference that it causes to a heavy traffic cell. This algorithm consists of two steps; solving the subchannel allocation according to proportional fair scheduling, then solving the power allocation. The advantage of their proposed scheme is that it takes into consideration the spatial traffic variation. However, the converging time might be too long. Moreover, designing a cooperative 
scheme in dense HetNet systems is usually prohibitive.

In [26], the authors proposed a cross-layer interference management technique based on the orthogonal random beamforming technology [27] and aided by the maxthroughput scheduler. In their scheme, the MBS adaptively selects a subset of beams according to its users' geometries, thereby creating spatial opportunities. These opportunities are to be exploited by the smaller BSs to access the same spectrum. In a multiple input single output (MISO) structure with $N_{t}$ transmit antennas, the precoding orthogonal matrix ${ }^{1} \mathbf{W} \in \mathbb{C}^{N_{t} \times Q}$ could support up to $Q=N_{t}$ independent data streams (i.e. serving $N_{t}$ macro users using one radio resource). However, due to multi-user interference ${ }^{2}$, it is sometimes optimal to have $Q<N_{t}$, especially when the total number of users is low or their SNR is high.

Considering downlink, authors in [26] introduced and analyzed a subset beam selection strategy in HetNet systems, with the objective of maximizing the throughput of each layer. Consider a large number of macro users and a chosen subset beam strategy $\mathcal{Q} \subseteq \mathcal{W}$, where $\mathcal{W}$ is the set of all possible beamforming strategies. There will be a macro user $k$ who maximizes the achievable rate over beam $i \in \mathcal{Q}$. Thus, at a given time, and over a given subchannel, the sum rate of the multiple streams using subset strategy $\mathcal{Q}$ is $R(\mathcal{Q})=\sum_{i \in \mathcal{Q}} \log _{2}\left(1+\max _{k \in \mathcal{K}} \Gamma_{k, i}(\mathcal{Q})\right)$, where $\Gamma_{k, i}(\mathcal{Q})$ is the $\operatorname{SINR}$ of user $k$ in beam $i$ in the case where the $\mathcal{Q}$ subset strategy is selected. Then the optimal subset strategy would be $\mathcal{Q}^{*}=\arg \max _{\mathcal{Q}} R(\mathcal{Q})$. Although the authors neatly formulated the beam selection problem, they did not provide a systematic method of solving it, giving the impression that they will go through all possible combinations, which is quite prohibitive since there are $\sum_{Q=1}^{N_{t}}\left(\begin{array}{c}N_{t} \\ Q\end{array}\right)$ different beamforming strategies.

\footnotetext{
${ }^{1} \mathbf{W}$ is a random matrix with orthogonal columns. $Q$ varies depending on which subset strategy $\mathcal{Q}$ is in use.

${ }^{2}$ Since the BS is transmitting multiple streams over the same resource, those beams interfere with each other. It is also called the quantization error.
} 
Exploiting the converse opportunity, the authors further proposed an opportunistic channel access scheme at the SBS level, aided with a power control mechanism. It has been shown that the interference from the macrocell to small cells statistically decreases as the number of macrocell beams $Q$ decreases. Therefore, $Q$ orthogonal directions in the $N_{t}$-dimensional space will be occupied by macrocell beams, while $N_{t}-Q$ directions will be free to be exploited by SBSs.

The optimum partial spectrum sharing in HetNets, which maximizes the spatial spectrum reuse, has been investigated in [28]. The objective was to maximize the total number of simultaneous transmissions within a given area. Authors considered a HetNet in which part of the spectrum is shared between the macrocell and small cells, while the other part is exclusively assigned to the macrocell. Thus, using such partial co-channel sharing and considering unplanned SBSs deployment, they found the ergodic capacity for both the macrocell and the small cells. Then, they found the area spectral efficiency (ASE), which is defined as the sum of the achievable rates per unit bandwidth, per unit area. They further addressed the criteria of the optimum partial spectrum sharing for ASE maximization. Moreover, the effects of the propagation environment, QoS requirements, as well as the density of small cells are all investigated. In their model, however, they did not consider the shadowing effect or small scale fading.

An orthogonal spectrum access strategy was proposed in [29] for a free crosslayer interference. In this scheme, the whole spectrum was partitioned into two orthogonal parts: one is to be used by the MBS and the other is to be shared among SBSs. To alleviate co-layer interference, the authors further suggested that each SBS randomly accesses a subset of the spectrum portion that has been allotted for SBSs use. This frequency domain ALOHA-like spectrum access is motivated by the hypothesis that neighbouring SBSs are unlikely to consistently access the same bands. Under this setup, authors found ASE for both macrocell and small cell networks, and 
they investigated ASE behaviour with respect to the density of small cells. It has been found that ASE saturates beyond a certain density threshold. Moreover, if the density is low, the maximum ASE is attainable when each SBS accesses the whole spectrum assigned to small cells. On the other hand, each SBS should access a decreasing portion of their spectrum as the density increases. For both layers, and under certain QoS requirements, authors have also investigated the criteria of partitioning the entire spectrum. They have shown that if the required QoS is uniform over the whole network, more than $90 \%$ of the spectrum should be assigned to the macrocell. However, an equal division of the spectrum would be the right partitioning when SBSs are to provide significantly higher data rates. Furthermore, it has also been shown that a channel aware scheduler at the macrocell level can provide significant ASE gains compared to a channel blind round robin scheduler, under the same QoS requirements.

To suppress the cross-tier interference to the MBS, interference mitigation strategies, in which SCUs adjust their maximum transmit power using open-loop and closedloop techniques, was proposed in [30]. In the open-loop control, a SCU adjusts the maximum transmit power so that the resulting cross-layer interference level is lower than a fixed threshold. On the other hand, to satisfy an adaptive interference threshold, the closed-loop control adjusts the maximum transmit power of the SCUs.

\subsection{Game Theoretic Approaches for Resource Al- location and Interference Management}

Considering only one radio resource for uplink, theoretical conditions that guarantee a peaceful coexistence of a MBS and a collection of closed access SBSs have been provided in [2]. Given a target macrocell SINR, the authors analytically characterized 
the small cells' maximum SINR target for which all transmit powers of small cell users are nonnegative. Under the assumptions of zero co-layer interference and a common target SINR across small cells, they found that the sum of decibel macrocell SINR and decibel small cell SINR is equal to a channel dependant constant denoted as the link budget. They further found that this link budget monotonically increases with path loss exponents. Moreover, the authors proposed a distributed game-based solution for cross-layer interference. The small cell utility function consists of a SINR dependant reward component and a penalty component, which is proportional to the interference caused at the MBS. Thus, small cells are discouraged of transmitting with high power. Nevertheless, there are still cases in which the required macrocell SINR cannot be attained. To solve this issue, the authors proposed a correction algorithm that ensures the macrocell QoS remains unaffected by the SBSs. They assume that the interfering channel from a SCU to a MBS is known to the SBS. This assumption is usually difficult to realize in frequency division duplex (FDD) systems. Moreover, co-layer interference was not thoroughly studied in [2].

An energy-efficient power allocation scheme was proposed in [31], considering spectrum sharing between a heterogeneous cognitive cellular network with small cells and several primary networks. The energy-efficient power allocation problem in heterogeneous cognitive radio networks with femtocells was formulated as a Stackelberg game. Then, a gradient-based iteration method was introduced to obtain the Stackelberg equilibrium. However, the spectrum resources were allocated orthogonally among the heterogeneous cognitive nodes by the cognitive MBS, meaning a centralized scheduling scheme. A broadband link is also required between MBS and each SBS, which is not required in our fully distributed proposed schemes. Furthermore, the spectrum resource assigned to the cognitive node (MU or SBS) cannot be shared with the other nodes. In [31], spectrum sharing is only between a heterogeneous cognitive node and a primary node. 
Authors in [32] borrowed the interference power constraint concept from cognitive radio networks to design price-based resource allocation strategies for the uplink transmission. MBS controls the transmit power of small cell users by pricing their resulting interference power levels at the MBS receiver, subject to a maximum tolerable interference margin. In this work, a Stackelberg game is formulated to maximize the revenue of the MBS (leader) as well as the individual utilities of the SBSs (followers). In particular, the interference tolerance margin at the MBS is used as the resource that the MBS and the SBSs compete for. With this game, simple pricebased resource allocation strategies are obtained. The formulation is as follows: let the aggregate interference from all SCUs that can be tolerated by MBS be $Q$, where the MBS problem is:

$$
\underset{\mu \succeq \mathbf{0}}{\operatorname{maximize}} \sum_{n=1}^{N} \mu_{n} I_{n}\left(p_{n}\right)
$$

subject to

$$
\sum_{n=1}^{N} I_{n}\left(p_{n}\right) \leq Q .
$$

where $I_{n}$ denotes the interference power at MBS received from $n^{\text {th }} \mathrm{SCU}$, and $\mu_{n}$ is the interference price assigned by MBS on $n^{t h}$ SCU. The objective function is the MBS utility function that represents its revenue. The constraint in the above problem is known as the interference temperature constraint [33]. On the other hand, the small cells problem is:

$$
\begin{aligned}
& \underset{p_{n} \leq 0}{\operatorname{maximize}} \lambda_{n} \log \left(1+\gamma_{n}\right)-\mu_{n} I_{n}\left(p_{n}\right) \\
& \text { subject to } \\
& p_{n} \geq 0 .
\end{aligned}
$$


where $\lambda_{n}$ is the utility gain per unit transmission rate for user $n$, and $\gamma_{n}$ is the SINR at $\operatorname{SBS} n$. Again, the objective function is the utility function of SCU $n$. The above two problems form a Stackelberg game. The Stackelberg equilibrium (SE) can be obtained as follows: let $\mu^{*}$ be a solution to the MBS problem and $p_{n}^{*}$ be a solution for $n^{\text {th }}$ SBS. For a given $\mu$, the SBS problem is solved first. Then, with the obtained best response function $p^{*}$ of the small cells, the MBS problem is solved for the optimal interference price of $\mu^{*}$.

The authors proposed two pricing schemes: uniform and non-uniform pricing. In the former pricing scheme, a uniform price is assigned to all SBSs, and in the latter scheme, different prices are established for different SBSs, based on the interference power. The equilibrium of the game has been studied in two network settings: sparsely deployed networks (rural areas) and densely deployed networks (urban areas). It should be noted that there are several drawbacks to this proposal. First, the formulation is brought from cognitive radio networks, in which the primary operator is different from the secondary operator. It therefore benefits from a real revenue by pricing the secondary users. On the other hand, in this proposal the MBS receives a virtual revenue from the small cells since they belong to the same operator all along. Thus, the MBS pricing on SCUs may restrict some SCUs who could potentially increase the network revenue. The second shortcoming is the requirement that all SBSs be connected to the MBS since the prices are computed at the MBS, and are then distributed to the small cells via high speed backhaul links. Further, in this work the co-layer interference was not studied thoroughly. 


\section{Chapter 4}

\section{Resource Allocation and Interference Management in HetNets with Moderate Number of Small Cells}

In general, controlling co-channel interference can be achieved via controlled spectrum sharing (SS) or spectrum avoidance $(\mathrm{SA})$. In this chapter, we propose a resource allocation and interference control scheme for HetNet systems by applying two-level algorithms. The overlay algorithm is motivated by a subtle fact: although SBSs are spectrally and energetically more efficient than MBSs, operators are sometimes obliged to use MBSs in order to serve mobile and isolated macrocell users (MUs) [2]. From the operators viewpoint, there are several reasons why it is important to satisfy MUs. First, the main role of the mobile network is to provide anytime anywhere service, especially for mobile/isolated users. Second, unlike SBSs, which are usually installed by the end users for their own interests, the MBS is installed by the operators themselves. According to the operators, MBS is modelled as a primary infrastructure, and satisfying users associated with MBS is a priority. Third, small cell users (SCUs) have many advantages over MUs. For example, transmitter-receiver separation is relatively very short in small cells. The frequency resources share of one 
SCU is relatively high due to the low number of SCUs per small cell. In addition, since the SCU owns the SBS, it may act selfishly to maximize its QoS; it may even tune the SBS settings and select its location to attain better service. Hence, operators are conflicted between achieving commitment towards MUs and maximizing network efficiency by relying heavily on small BSs.

It has been indicated in [38] that a multiple access channel potentially provides greater channel capacity than allowing several nodes, which are located within the same macrocell, to simultaneously share a common channel ${ }^{1}$. We resolve the mentioned conflict by formulating an optimization problem in which the MBS is given full access to the whole pool of system resources, in order to exclusively select the minimal number of subchannels fairly satisfying the MUs, provided that this minimal number does not exceed a predefined upper bound.

The underlay algorithm, which runs at each SBS in a distributed fashion, is proposed to control the co-layer interference using an effective solution; namely, the non-cooperative game theory $[10,39]$. The motivation of this approach is as follows. Co-channel interference in its essence is a result of the competition between different transmitters over the network resources (e.g. bandwidth and energy). For such a conflict, non-cooperative game theory is considered a natural strategic solution, where such a contention can be modelled as a game whose players are the transmitters in adjacent small cells.

In general, this chapter aims to propose a holistic and fully distributed solution for energy-efficient resource allocation and inter-cell interference management in HetNets. In addition to the improvements in the aggregate spectral efficiency, as well as the border user spectral efficiency achieved by the proposed solution, it can substantially improve energy efficiency. It can also reduce the complexity of the resource

\footnotetext{
${ }^{1}$ In our case, those nodes are either MBS or a collection of SBSs.
} 
allocation process. The decentralized strategy, aided by game-based interference control, could allow for dynamic soft frequency reuse within each macrocell; it may also lead to a plug and play SBS.

The rest of this chapter is organized as follows. The system model and assumptions are introduced in Section 4.1. In Section 4.2, the optimization problem of macrocell assignment is formulated and near-optimally solved by the proposed successive algorithm. Section 4.3 formulates, analyzes, and solves the underlay game problem, which deals with the co-layer interference among small cells. Then, simulation results are shown and discussed in Section 4.4. Finally, this work is concluded and future work is suggested in Section 4.5.

\subsection{System Description}

In the heterogeneous network shown in Fig. 1.1, we consider a discretized time, orthogonal frequency division multiplexing (OFDM) system. The considered macrocell area contains $N$ SBSs and their associated SCUs as well as one MBS and its associated $J$ MUs. The set of perfectly orthogonal OFDM subchannels available in this area is $\mathcal{M}=\{1, \ldots, m, \ldots, M\}$. Similar to $[2,41]$, our proposed interference control scheme does not require specific type of medium access control (MAC) in small cells, rather the MAC protocol in SBSs can be chosen arbitrarily. Thus, in this research, we chose the time division multiple access (TDMA) as the SBSs' MAC protocol. The only reason for this choice is to simplify the description of our proposed scheme. Hence, for a given time slot $t$, we assume one active user per small cell.

The sets of all SBSs and active SCUs, are $\mathcal{N}=\left\{S B S_{1}, S B S_{2}, \ldots, S B S_{N}\right\}$ and $\mathcal{N}_{S C U}=\left\{S C U_{1}, S C U_{2}, \ldots, S C U_{N}\right\}$, respectively. In the macrocell, however, we will design an OFDMA-based scheduling scheme that facilitates a simultaneous and spectrally orthogonal access of $J$ MUs. Thus, the set of all MUs is 
$\mathcal{J}=\left\{M U_{1}, M U_{2}, \ldots, M U_{J}\right\}$, and the index of their MBS is 0 . As briefly described in the introduction, cross-layer interference would be avoided by SBSs since they would access their own band or opportunistically access remaining subchannels, which aren't used by MBS. Considering uplink, if subchannel $m$ is available at the small cells, the signal to interference plus noise ratio (SINR) over the $m$ th subchannel, at $S B S_{n}$, and in time slot $t$ would be

$$
\begin{aligned}
\gamma_{n m}(t) & =\frac{p_{n m}(t) h_{n n m}(t)}{\sum_{\substack{k \in \mathcal{N} \\
k \neq n}} p_{k m}(t) h_{n k m}(t)+\sigma_{n m}^{2}} \\
& =\frac{p_{n m}(t) h_{n n m}(t)}{I_{n m}(t)+\sigma_{n m}^{2}},
\end{aligned}
$$

where $\gamma$ is SINR. $p_{n m}$ and $p_{k m}$ are the transmit power over the $m$ th subchannel from the associated user $S C U_{n}$ and the interfering user $S C U_{k}$, respectively. In general, $h_{n k m}$ is the $m$ th subchannel gain from $S C U_{k}$ in the $k$ th cell to $S B S_{n}$ located at cell $n$. Hence, $h_{n n m}$ and $h_{n k m}$ are respectively the power gains of the desired channel from $S C U_{n}$ to $S B S_{n}$, and the interfering channel from $S C U_{k}$ located at cell $k$ to $S B S_{n}$. In an abuse of notation, we may use the shorthand $h_{n m}$ to refer to the $m$ th subchannel gain between $S C U_{n}$ and its serving BS $S B S_{n}$ (i.e., $h_{n n m}$ ). $I_{n m}$ is the total interference at $S B S_{n}$. In addition, $\sigma_{n m}^{2}$ is the power of the additive white Gaussian noise at $S B S_{n}$. On the other hand, if subchannel $m$ is used by MBS and assigned to $M U_{j}$, then the SINR at MBS in time slot $t$ would be

$$
\gamma_{j m}(t)=\frac{p_{j m}(t) h_{0 j m}(t)}{\sigma_{0 m}^{2}} .
$$

Again, we may use the shorthand $h_{j m}$ to refer to $h_{0 j m}$. Since the proposed interference scheme depends only on the current time slot, for simpler presentation, the argument $t$ will be omitted in the subsequents.

For every $m \in \mathcal{M}$, the value of the desired channel is assumed known to the 
receiving BS. Moreover, since the physical layer transmission scheme in this system is OFDM, each subchannel is assumed flat during each time slot. Finally, we assume that BSs make decisions and dictate those decisions on their associated users whose data queues are assumed nonempty.

\subsection{Macro-BS Overlay Optimization Problem}

In this section, we first formulate the overlay problem in the MBS, and we define the variables and parameters. Since the formulated problem is analytically hard to solve, we then introduce the overlay algorithm that solves it numerically.

\subsubsection{Problem Formulation}

In order to satisfy macrocell users within certain limits, and simultaneously maximize the network efficiency, we need to carefully select the MUs' subchannels. It is reasonable that a minimal number of subchannels, which guarantee the required quality of service (QoS), are first exclusively assigned to the macrocell users, provided that this minimal number should not exceed an operator-defined ceiling. The remaining subchannels $\left(\mathcal{M}^{S}\right)$ should then be left to small cells to help maximize the network sum rate. Therefore, the following optimization problem, whose solution is to be found by the MBS, is proposed, 


$$
\begin{aligned}
& \underset{X_{j m}, p_{j m}}{\operatorname{minimize}} \sum_{j \in \mathcal{J}} \sum_{m \in \mathcal{M}}\left(p_{\text {total }}^{(C)} X_{j m}+p_{j m}\right) \\
& \text { subject to } \\
& \sum_{m \in \mathcal{M}} B \log _{2}\left(1+\frac{p_{j m} h_{j m}}{\beta_{j} \sigma_{0 m}^{2}}\right) X_{j m} \geq R_{j}, \forall j, \\
& \sum_{m \in \mathcal{M}} p_{j m} \leq p_{\text {total }}^{(C)}, \forall j, \\
& \sum_{j \in \mathcal{J}} X_{j m} \leq 1, \forall m, \\
& \sum_{m \in \mathcal{M}} \sum_{j \in \mathcal{J}} X_{j m} \leq\lceil\xi M\rceil, \\
& X_{j m} \in\{0,1\}, \forall m \& j, \\
& p_{j m} \geq 0, \forall m \& j,
\end{aligned}
$$

where $p_{\text {total }}^{(C)}$ is the maximum transmit power allowed for each MU at every given time, and $X_{j m}$ is a binary variable which takes a value of 1 if the $j^{\text {th }}$ MU is assigned the $m^{\text {th }}$ subchannel, and 0 otherwise. $B$ is the bandwidth of each subchannel, $\beta_{j}=\frac{-\ln \left(5 B E R_{j}\right)}{1.5}$ is a positive constant that takes into account the required bit error rate (BER) of the application under transmission. $R_{j}$ is the minimum data rate required by $M U_{j}$. $\xi$ is an operator-defined parameter. Problem (4.3) is optimized over the variables $X_{j m}$ and $p_{j m}$ for all $m$ and $j$. The objective function is meant primarily to minimize the number of subchannels used by MUs. Since communicating with MBS is naturally less energy efficient than that with SBSs, the objective function is further designed to support energy saving policy by adding the second term. However, minimizing the number of assigned subchannels has higher priority than minimizing the power consumption. This higher priority is achieved via multiplying the first term in the objective function by an expensive factor (i.e., $p_{\text {total }}^{(C)}$ ). Constraint (4.3a) makes sure 
that the minimum required QoS of each MU is attained. Moreover, constraint (4.3b) takes into account the fact that each MU has a limited power budget $p_{\text {total }}^{(C)}$. Free intra-cell interference is ensured by constraint (4.3c), where each subchannel $m$ cannot be assigned to more than one MU. Constraints (4.3d) ensures that the number of resources assigned to MUs does not exceed a predefined number since some resources should be left for small cells users. Constraints (4.3e) and (4.3f) specify the spaces of the variables $X_{j m}$ and $p_{j m}$ respectively. Note that the MBS has full access to the whole resources (i.e., $\mathcal{M}$ ) in order to minimize the resources assigned to MUs to the least possible number by harvesting all frequency diversity. Additionally, this assignment is dynamic because the selected subchannels by MBS changes from one time slot to another depending on the environment conditions.

The above program effectively divides the set of radio resources $\mathcal{M}$ into two disjoint sets: $\mathcal{M}^{M}$ and $\mathcal{M}^{S}$ such that $\left|\mathcal{M}^{M}\right| \leq\lceil\xi M\rceil$ and $\left|\mathcal{M}^{S}\right|=\left(M-\left|\mathcal{M}^{M}\right|\right)$. The first set is assigned to the macrocell while the second set is to be shared by small cells. $\xi$ is a partitioning factor chosen by the operator, and generally depends on two factors. First, $\xi$ is proportional to the ratio of the number of MUs to the number of SCUs. Second, $\xi$ depends on how far the operator is willing to satisfy the MUs by compromising the network efficiency. Lower values of $\xi$ would increases the network efficiency (i.e., higher profit) while higher values of $\xi$ would enhance the probability of MUs satisfactions (i.e., maintaining good reputation). For example, one way is to set $\xi=\frac{1}{2}\left(\eta+\frac{J}{\text { Total no. of users }}\right)$, where $\eta \in[0,1]$ is a factor of how far the operator desires to satisfies the MUs.

\subsubsection{Overlay Algorithm in MBS}

The optimization problem in (4.3) is a mixed integer non-linear programming (MINLP) whose complexity level is NP-hard [42]. It is analytically difficult, and hard to find its solution in polynomial time. Fortunately, the above problem has 
especial characteristics. In particular, to guarantee the macrocell users QoS by using minimum resources, the assigned subchannels of each MU should be as powerful as possible.

When we have several users and several subchannels, the best assignment method is the Hungarian algorithm on the matrix $H_{J X M}=\left[h_{j m}\right]$ [43]. Indeed, Hungarian algorithm guarantees optimality if the number of users equals the number of subchannels, while it provides a good suboptimal solution in case the number of users is not equal to the number of subchannels [22]. Therefore, we propose Algorithm 1, which finds a good suboptimal solution of (4.3) by successively applying Hungarian algorithm on the channel matrix $H_{J X M}=\left[h_{j m}\right]$, to approximately find minimal assignment. Then, it solves a closely related convex optimization problem using the simple Karush-Kuhn-Tucker (KKT) technique to distribute a minimum amount of power over the assigned subchannels. Note that, in case $\lceil\xi M\rceil$ subchannels have been assigned to the MUs while some MUs are not yet satisfied, then they should pour their full power using waterfilling scheme to get the best effort service.

After some initialization steps, Algorithm 1 iterates over two main steps; Hungarian assignment and testing MUs' satisfactions. Below we summarize how Algorithm 1 works.

\section{First: initialization}

1. A varying set $\mathcal{J}_{t}$ of cardinality $J_{t}$ is created. $\mathcal{J}_{t}$ contains the indices of unsatisfied MUs, and hence, It initially equals $\mathcal{J}$ with cardinality $J$.

2. $J$ empty sets $\left\{\mathcal{M}_{j}\right\}_{j=1}^{J}$ are created and associated with each MU. $\mathcal{M}_{j}$ is to contain the indices of the assigned subchannels to $M U_{j}$.

3. A varying matrix $H_{t}$ is created whose initial value is $H=\left[h_{j m}\right]$. 


\section{Second: iteration}

1. Hungarian algorithm is applied on $H_{t}$, where each unsatisfied MU would be assigned the best subchannel from its perspective. If one subchannel is seen the best by two MUs, Hungarian algorithm will assign it to the MU that best utilizes it.

- The columns corresponding to the assigned subchannels are removed from $H_{t}$. This is to avoid assigning them again in future iterations.

- The index of the subchannel that has been assigned to $M U_{j}$ is placed into $\mathcal{M}_{j}$.

2. Each unsatisfied MU $j \in \mathcal{J}_{t}$ will be checked whether it is now satisfied with its current resources $\mathcal{M}_{j}$. This is done by testing the feasibility of the following problem:

$$
\begin{aligned}
& \underset{p_{j m}}{\operatorname{minimize}} \sum_{m \in \mathcal{M}_{j}} p_{j m} \\
& \text { subject to } \\
& \sum_{m \in \mathcal{M}_{j}} B \log _{2}\left(1+\frac{p_{j m} h_{j m}}{\beta_{j} \sigma_{0 m}^{2}}\right) \geq R_{j} \\
& \sum_{m \in \mathcal{M}_{j}} p_{j m} \leq p_{\text {total }}^{(C)} \\
& p_{j m} \geq 0, \forall m \in \mathcal{M}_{j}
\end{aligned}
$$

If problem (4.4) is feasible for $M U_{j}$, then:

- $\mathrm{MU} j$ is removed from set $\mathcal{J}_{t}$, and the $j^{\text {th }}$ row is removed from $H_{t}$. This is to make sure that $M U_{j}$ will not be assigned more than what he needs. 
- The convex problem (4.4) is solved for $M U_{j}$.

3. The iteration part repeats until $\mathcal{J}_{t}$ becomes empty or the number of assigned resources reached its limit: $\sum_{j \in \mathcal{J}}\left|\mathcal{M}_{j}\right|=\lceil\xi M\rceil$.

It is easy to verify that the constraints in (4.4) constitute a convex set, and the Slater's condition is satisfied. Hence, the solution to this problem is given by

$$
p_{j m}^{*}= \begin{cases}\frac{B \mu_{1}}{\ln 2\left(1+\mu_{2}\right)}-\frac{\beta j \sigma_{0 m}^{2}}{h_{j m}}, & h_{j m} \geq \frac{\beta_{j} \sigma_{0 m}^{2}\left(1+\mu_{2}\right) \ln 2}{B \mu_{1}} \\ 0 & \text { Otherwise }\end{cases}
$$

where $\mu_{1}$ and $\mu_{2}$ are KKT multipliers. It is observable that Hungarian algorithm selects the strongest channels for $M U_{j}$ in an incremental descending fashion. If problem (4.4) is feasible, then all channels $h_{j m} \forall m \in \mathcal{M}_{j}$ are used (i.e., $p_{j m}^{*}>0$ ). Furthermore, for achieving minimum power, constraint (4.4a) would always be active (i.e., with strict equality). Accordingly, satisfying $M U_{j}$ may be accomplished either with partial power usage (i.e., constraint $(4.4 \mathrm{~b})$ is inactive) or with full power usage (i.e., constraint (4.4b) is active). After manipulating KKT conditions, the following is concluded.

- When constraint (4.4b) is inactive, then $\mu_{2}=0$ and $\mu_{1}=2^{C_{1}}$, where

$$
C_{1}=\frac{R_{j}}{\left|\mathcal{M}_{j}\right| B}-\frac{1}{\left|\mathcal{M}_{j}\right|} \sum_{m \in \mathcal{M}_{j}} \log _{2}\left(\frac{h_{j m}}{\sigma_{0 m}^{2}}\right)-\log _{2}\left(\frac{B}{\beta_{j} \ln 2}\right)
$$

In this case the feasibility condition would be $\sum_{m \in \mathcal{M}_{j}}\left(\frac{\mu_{1} B}{\ln 2}-\frac{\beta_{j} \sigma_{0 m}^{2}}{h_{j m}}\right)<p_{\text {total }}^{(C)}$.

- If, however, constraint (4.4b) is active, the feasibility condition would be $C_{2}=$ 
$2^{C_{1}}$, where

$$
C_{2}=\frac{\ln 2}{\left|\mathcal{M}_{j}\right| B}\left(p_{\text {total }}^{(C)}+\sum_{m \in \mathcal{M}_{j}} \frac{\beta_{j} \sigma_{0 m}^{2}}{h_{j m}}\right)
$$

In this case $\frac{\mu_{1}}{1+\mu_{2}}=C_{2}$. This value can be directly plugged into (4.5) to find the optimal power for each subchannel m assigned to $M U_{j}$. The condition of this case is quite strict due to the fact that achieving $R_{j}$ with exactly full power is very seldom to occur in a problem that minimizes the consumed power.

Since problem (4.4) is convex and the Slater's condition is satisfied, KKT conditions are simultaneously sufficient and necessary conditions. Therefore, if none of the above conditions are satisfied, then problem (4.4) is not feasible, and $M U_{j}$ needs to be assigned more subchannels.

While the MBS is updating $M U_{j}$ with its set of assigned subchannels $\mathcal{M}_{j}$, each SBS listens to this information and makes a note of it. After MBS executed its algorithm, SBSs conclude that their set of accessible subchannels at time slot [t] is $\mathcal{M}^{S}(t)=\mathcal{M} \backslash \cup_{j \in \mathcal{J}} \mathcal{M}_{j}(t)$

\subsection{Formulation of a Non-Cooperative Game}

In this section, we treat the co-layer interference among small cells over the $m^{\text {th }}$ subchannel. This treatment is applicable to all $m \in \mathcal{M}^{S}(t)$. A game problem among all small cells is formulated in the next subsection. Then, the game utility function is designed in Subsection 4.3.2. After that, the equilibrium is analyzed in Subsection 4.3.3, followed by a discussion on the game outcome in Subsection 4.3.4. Next, an algorithm that converges to the equilibrium point in a distributed fashion is proposed in Subsection 4.3.5. Finally, two forms of the game, which differ in the actions space available to each user, are suggested in Subsection 4.3.6. 


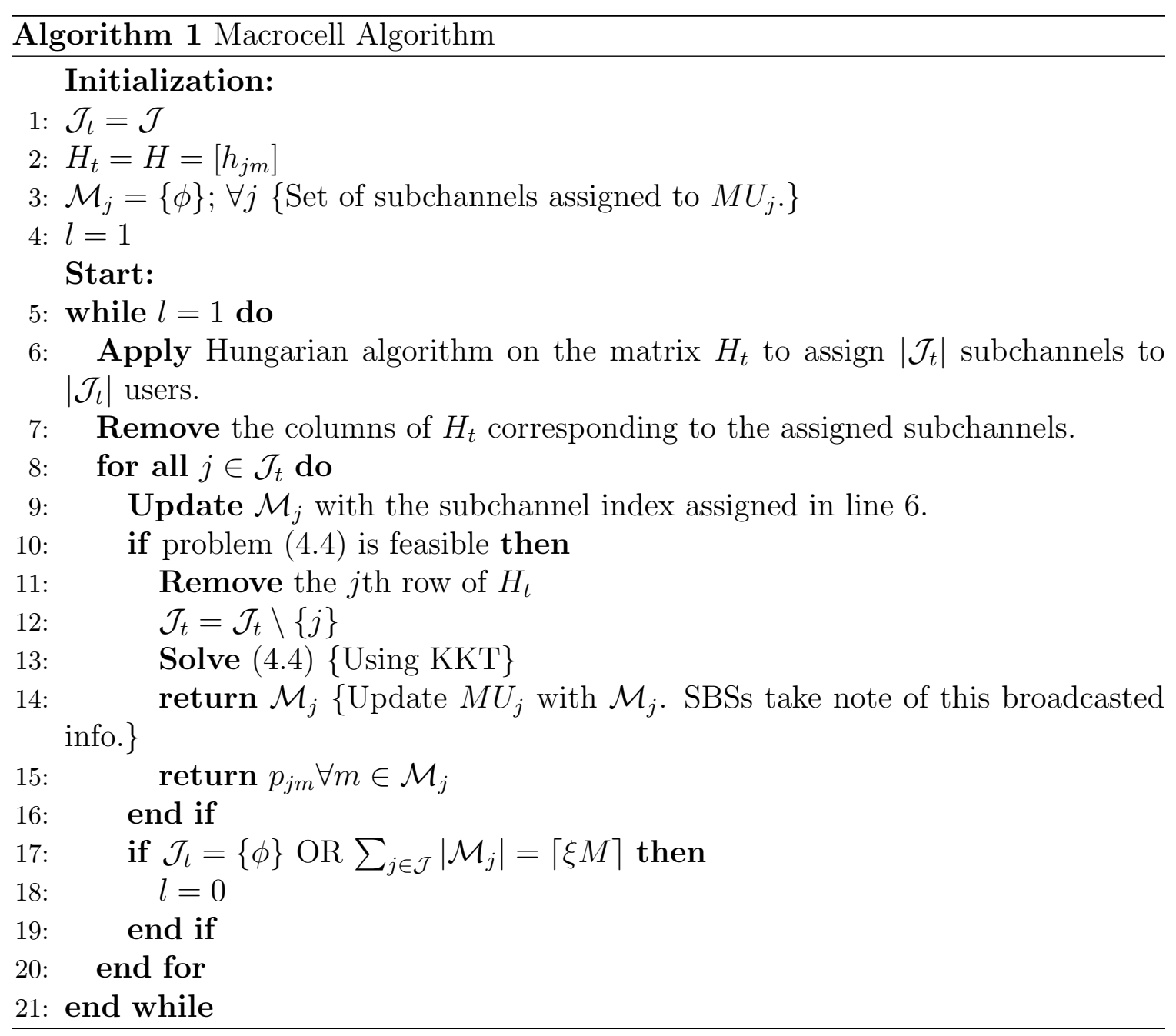

\subsubsection{Non-Cooperative Game Among SBSs}

Small cell users (SCUs), which are located in adjacent small cells and operate on the same subchannel $m \in \mathcal{M}^{S}$, mutually affect each other through inter-cell interference. Such users' interaction can be modelled as a game whose players are the receiving SBSs that take decisions and command their SCUs to act accordingly. Therefore, we propose the game $\mathcal{G}=\left(\mathcal{N},\left\{\mathcal{P}_{n m}\right\}_{n=1}^{N},\left\{u_{n m}\right\}_{n=1}^{N}\right)$, where:

- $\mathcal{N}=\left\{S B S_{1}, S B S_{2}, \ldots, S B S_{N}\right\}$ is the set of SBSs that receive over the same subchannel $m \in \mathcal{M}^{S}$.

- $\mathcal{P}_{n m}=\left\{p_{n m}: p_{n m} \in\left[0, p_{n m}^{\max }\right]\right\}$ is a convex set of transmit power that 
represents the space of actions available to $S C U_{n}$ on subchannel $m$, and

$p_{n m}^{\max } \geq 0$, is the maximum transmit power. Furthermore, the action profile $\mathbf{p}=\left(p_{1 m}, p_{2 m}, \ldots, p_{N m}\right) \in \mathcal{D}_{m}$ denotes simultaneous actions of all players, where $\mathcal{D}_{m}$ is the product space $\mathcal{P}_{1 m} \times \mathcal{P}_{2 m} \times \ldots \times \mathcal{P}_{N m}$.

- $u_{n m}$ : is the $n$th user's utility on subchannel $m$.

The choice of the utility function has a great influence on the nature of the game and on the behaviors of the players. Therefore, the following subsection is dedicated to introduce and analyze the proposed utility function.

\subsubsection{Utility Function}

From a wireless communications viewpoint, utility function is a mathematical expression that quantifies the level of satisfaction a user obtains by using the system resources. In designing a given game, there are different possible utility functions, each of which reflects a set of rules governing that game. In fact, a good candidate for the utility function is the one that places the elements of the actions set $\mathcal{P}$ in a desired order. In the following, the game preference relations that are specific in our interference management problem are identified and then the utility function that represents this structure is introduced.

Delay and bit error rate (BER) are the main factors that affect the quality of wireless services. Low data rate and frequent retransmissions, which are essentially due to weak SINR, are the main reasons of delay. Moreover, weak SINR increases the BER, which in turn degrades the level of clarity, especially in voice services. Obviously, the desired channel and interference play a crucial role in affecting the received SINR, service quality and hence the level of user's satisfaction. Therefore, when the main objective is maximizing spectral efficiency, defining the user's utility as a concave increasing function of the user's SINR is a preferable option [39,44-46]. 


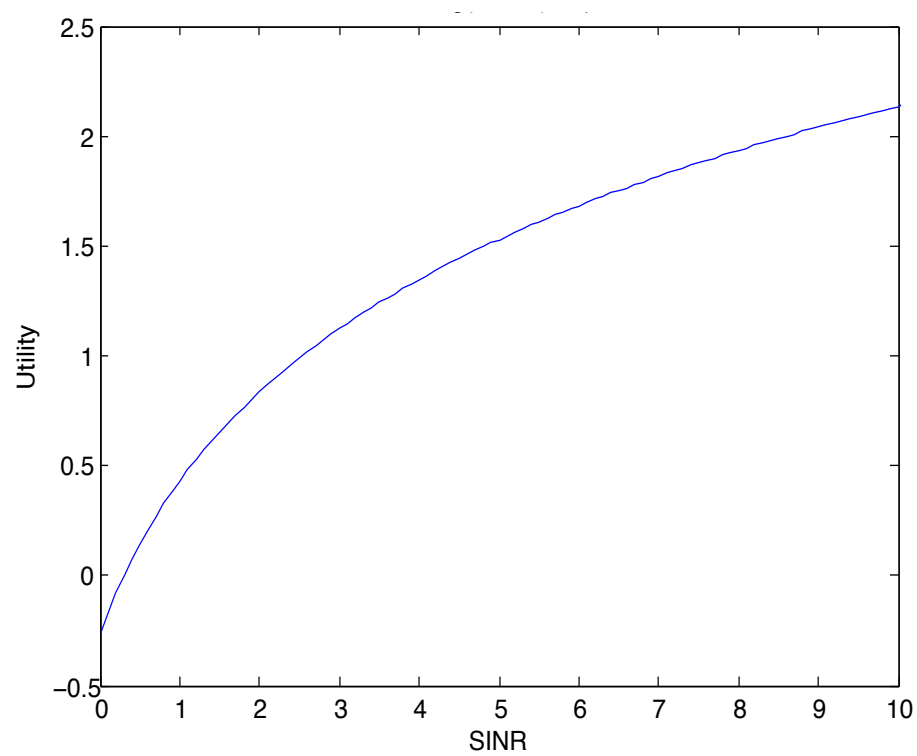

(a)

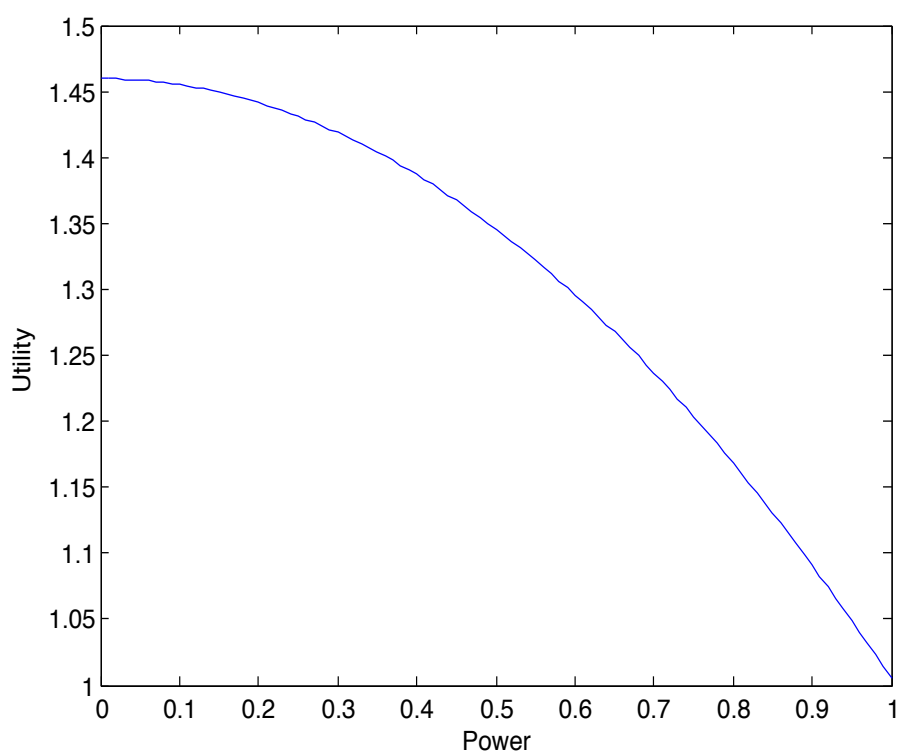

(b)

Figure 4.1: Utility function: a) with respect to SINR while power is fixed, and b) with respect to power while SINR is fixed.

To this end, any function that is proportional to the logarithmic Shannon capacity may be a good choice. However, since such utility would be directly proportional to the transmit power, the best way to maximize the utility value for each user in a noncooperative game is by transmitting with the highest possible power. This behaviour will adversely increase the level of interference in the network and hence reduce the spectral efficiency. Consequently, this function is not a favourable utility function. To correct it, a penalty term (or cost) should be incorporated to it so that it prevents users from always transmitting at full power. Thus, if a user's SINR is fixed, then the utility function should be a decreasing function with the transmit power. For subchannel $m$, the following user's utility function is devised for the on-hand game $\mathcal{G}:$

$$
u_{n m}\left(p_{n m}, \gamma_{n m}\right)=\alpha_{n} \ln \left(1+\frac{\gamma_{n m}}{\beta_{n}}\right)-\lambda_{n m} p_{n m}^{2}
$$


where $\alpha_{n}$ is a fairness weighting factor that ensures end users are getting relatively fair share of the channel. It is the reciprocal of the exponential moving average $(E M A)$ of the achieved rates;

$$
1 / \alpha_{n}=E M A_{n}(t)=a \cdot R_{n}(t)+(1-a) \cdot E M A_{n}(t-1),
$$

where $a \leq 1$ is a positive constant that determines the depth of memory of the EMA, and $R_{n}(t)$ is the achieved rate at time slot $t$. Furthermore, $\beta_{n}=\frac{-\ln \left(5 B E R_{n}\right)}{1.5}$, and $\lambda_{n m}$ is the pricing factor for user $n$ on subchannel $m$, measured in $\frac{n p s / H z}{\text { watt }^{2}}$. In case of uniform pricing factor $\lambda$ across users, cell edge SCU would unfairly incur a higher cost. This would be due to its legitimate need to transmit with a relatively high power to compensate its channel weakness and overcome the higher interference. On the other hand, central SCU, which has the privilege of strong channel and weak interference, can further increase its utility by increasing its transmit power. To correct this unfair situation, $\lambda_{n m}$ should be a location dependent factor such that as a user goes farther from its serving SBS, the price of every watt it radiates goes down. Thus, a low pricing factor would tempt cell edge SCU to transmit high, while a high pricing factor would restrict central user to transmit low. In this research, we will fully characterize the value of $\lambda_{n m}$, which takes into account this requirement.

Note that since $\gamma_{n m}$ is a function of $p_{n m}$ and $\mathbf{p}_{-n m}$ (where $\mathbf{p}_{-n m}=$ $\left.\left\{p_{1 m}, \ldots, p_{(n-1) m}, p_{(n+1) m}, \ldots, p_{N m}\right\}\right)$, then utility function can be written as $u_{n m}\left(p_{n m}, \mathbf{p}_{-n m}\right)$.

Since the following analysis and procedures are applicable to each subchannel $m \in \mathcal{M}^{S}$, we may omit the subscript $m$ in the subsequent unless it is really needed. Thus, assuming the game is played over the $m^{\text {th }}$ subchannel, our designed utility function in (4.6) possesses the following properties:

1. For a fixed transmit power $p_{n}$, 
(a) $\frac{\partial u_{n}\left(p_{n}, \gamma_{n}\right)}{\partial \gamma_{n}}=\frac{\alpha_{n}}{\beta_{n}+\gamma_{n}}>0$, i.e., the utility is an increasing function of SINR.

(b) $\frac{\partial^{2} u_{n}\left(p_{n}, \gamma_{n}\right)}{\partial \gamma_{n}^{2}}=\frac{-\alpha_{n}}{\left(\beta_{n}+\gamma_{n}\right)^{2}}<0$, i.e., the rate of the increasing utility is decreasing with respect to SINR. In other words, the higher the SINR, the slower the utility function (see Fig. 4.1(a)).

2. For a fixed SINR $\gamma_{n}$,

(a) $\frac{\partial u_{n}\left(p_{n}, \gamma_{n}\right)}{\partial p_{n}}=-2 \lambda_{n} p_{n}<0$, i.e., the utility is a decreasing function with respect to the transmit power.

(b) $\frac{\partial^{2} u_{n}\left(p_{n}, \gamma_{n}\right)}{\partial p_{n}^{2}}=-2 \lambda_{n}<0$, i.e., the rate of the decreasing utility is increasing with respect to the transmit power. In other words, as a SCU increases its transmit power, the utility function declines in a faster rate (see Fig. $4.1(\mathrm{~b}))$.

3. If the desired channel $h_{n n}$ and received interference $I_{n}$ are fixed, then $u_{n}\left(p_{n}, \mathbf{p}_{-n}\right)$ is a concave function of transmit power $p_{n}$. To prove this property, let us assume that $\alpha_{n} \ln \left(1+\frac{p_{n} h n}{\beta_{n}\left(I_{n}+\sigma_{n}^{2}\right)}\right)=\Psi_{n}\left(p_{n}, \mathbf{p}_{-n}\right)$ and $-\lambda_{n} p_{n}^{2}=\Omega_{n}\left(p_{n}\right)$. Now, since $\Psi_{n}\left(p_{n}, \mathbf{p}_{-n}\right)$ and $\Omega_{n}\left(p_{n}\right)$ are both concave functions of $p_{n}$, then their sum $u_{n}\left(p_{n}, \mathbf{p}_{-n}\right)=\Psi_{n}\left(p_{n}, \mathbf{p}_{-n}\right)+\Omega_{n}\left(p_{n}\right)$ must be also a concave function of $p_{n}[47]$.

Properties $1 . \mathrm{b}$ and $2 . \mathrm{b}$ are due to the fact that the utility function is a concave function of SINR and transmit power, respectively.

At this point, it is important to introduce the concept of marginal utility. Marginal utility is the additional satisfaction, or additional amount of utility, gained by each extra unit of consumption (i.e., marginal utility = derivative of the utility with respect to the consumption argument). There are two ways for a SBS to improve its utility: by improving SINR via transmitting more power, or by reducing the cost which is 


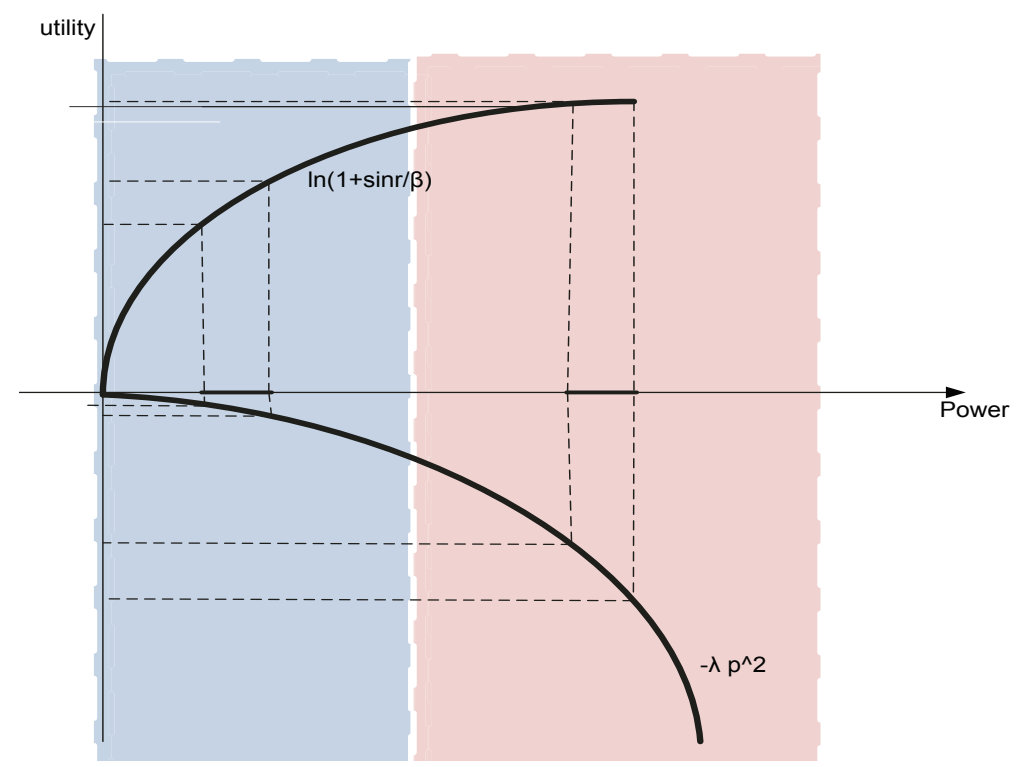

Figure 4.2: SINR utility and its cost in low and high power regimes.

done by transmitting with less power.

$$
u_{n}=\underbrace{\alpha_{n} \ln \left(1+\frac{\gamma_{n}}{\beta_{n}}\right)}_{\text {Improving utility through SINR }}+\underbrace{-\lambda_{n} p_{n}^{2}}_{\text {Improving utility through cost reduction }}
$$

For our utility function defined in (4.6), the marginal utility of SINR is $\psi_{n}=$ $\Psi_{n}^{\prime}\left(p_{n}, \gamma_{n}\right)=\frac{\alpha_{n}}{\beta_{n}+\gamma_{n}}$, while the marginal utility of holding (saving) the power is $\omega_{n}=$ $\Omega_{n}^{\prime}\left(p_{n}\right)=-2 \lambda_{n} p_{n}$. From property 1.b, the marginal utility of SINR decreases as SINR increases. Moreover, property 2.b tells us the marginal utility of saving power decreases as the transmitter gets more conservative in spending power. As depicted in Fig. 4.2, the marginal utilities decrease in the opposite direction with respect to the transmit power. The SINR marginal utility decreases as the power increases while the cost reduction marginal utility decreases as the transmit power decreases. This behavior suggests that there is a mid point at which SCU should transmit with. At this point, both marginal utilities would have the same value. Interestingly, after reaching that point, the utility is going to decrease if the SCU increases its 


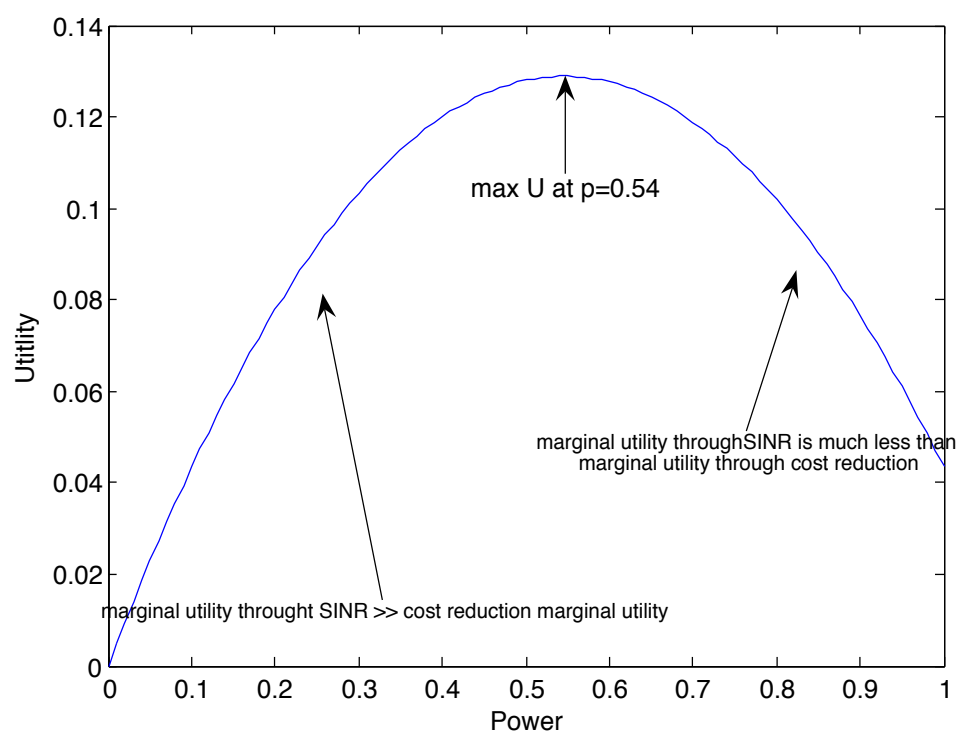

Figure 4.3: Utility function w.r.t power, when the desired channel $h$ and interference $I$ are fixed.

power. This is simply because the marginal utility of SINR is going to diminish while the additional cost is going to increase dramatically, causing the utility function to deteriorate as it is illustrated in Fig. 4.3 and Fig. 4.2. Moreover, if it decreases its power, the marginal utility of cost reduction diminishes in this direction while the loss in SINR is increasing. In summary, the utility function in (4.6) ensures that the level of service each SCU gets is worth exactly the price it pays, leading to a stable state.

\subsubsection{Equilibrium Analysis: Existence and Uniqueness}

With the game formulation described in the previous subsections, it is important to find the Nash equilibrium point, which represents the best transmit power for each SCU $n$ who uses subchannel $m$. To that end, we need to prove first whether the Nash equilibrium point of the formulated game exists or not. Therefore, we start off by introducing the following lemma. 
Lemma 1. There exist at least one Nash equilibrium point for the game $\mathcal{G}=$ $\left(\mathcal{N},\left\{\mathcal{P}_{n}\right\},\left\{u_{n}\right\}\right)$.

Proof. For every player $n \in \mathcal{N}$, we observe the following:

- The set of $p_{n}$ (i.e., $\mathcal{P}_{n}=\left\{p_{n}: p_{n} \in\left[0, p_{n}^{\max }\right]\right\}$ ) is convex set. In addition, it is closed, and bounded by $p_{n}^{\max }$; therefore, it is compact.

- Property (3) in the previous subsection shows that the utility function $u_{n}\left(p_{n}, \mathbf{p}_{-n}\right)$ is a concave function of the transmit power $p_{n}$ (see Fig. 4.3 as well).

- The utility function $u_{n}\left(p_{n}, \mathbf{p}_{-n}\right)$ is obviously a continuous function of the power vector $\mathbf{p}=\left[p_{n}, \mathbf{p}_{-n}\right]$.

Consequently, by using theorem (1) in [48], it straightforward follows that $\mathcal{G}=$ $\left(\mathcal{N},\left\{\mathcal{P}_{n}\right\},\left\{u_{n}\right\}\right)$ has at least one Nash equilibrium point.

To prove the uniqueness, we first present some required background and results taken from [48]. Let $\Phi(\mathbf{p}, \mathbf{r})=\sum_{n=1}^{N} r_{n} u_{n}(\mathbf{p})$ be a weighted non-negative sum of the functions $\left\{u_{n}\right\}_{n=1}^{N}$ for each non-negative vector $\mathbf{r} \geq 0 \in \mathbb{R}^{N}$. Moreover, for a fixed $\mathbf{r}$, the mapping $\mathbf{g}(\mathbf{p}, \mathbf{r}): \mathbb{R}^{N} \longmapsto \mathbb{R}^{N}$ is defined as:

$$
\mathbf{g}(\mathbf{p}, \mathbf{r})=\left[\begin{array}{c}
r_{1} \frac{\partial u_{1}(\mathbf{p})}{\partial p_{1}} \\
r_{2} \frac{\partial u_{2}(\mathbf{p})}{\partial p_{2}} \\
\vdots \\
r_{N} \frac{\partial u_{N}(\mathbf{p})}{\partial p_{N}}
\end{array}\right]
$$


$\mathbf{g}(\mathbf{p}, \mathbf{r})$ is called pseudogradient of $\Phi(\mathbf{p}, \mathbf{r})$, and in our case each element in the pseudogradient is:

$$
g_{n}(\mathbf{p}, \mathbf{r})=r_{n} \frac{\alpha_{n} h_{n n}}{\beta_{n}\left(I_{n}+\sigma_{n}^{2}\right)+p_{n} h_{n n}}-2 \lambda_{n} p_{n}
$$

Let the $N X N$ matrix $G(\mathbf{p}, \mathbf{r})$ be the Jacobian of $\mathbf{g}(\mathbf{p}, \mathbf{r})$ with respect to $\mathbf{p}$ for a fixed $\mathbf{r}>0$. That is, the $n$th column of $G(\mathbf{p}, \mathbf{r})$ is $\frac{\partial \mathbf{g}(\mathbf{p}, \mathbf{r})}{\partial p_{n}}, n=1,2, \ldots, N$. Then, the following lemma is easily deducible from Theorem (2) and Theorem (6) in [48].

Lemma 2. If the symmetric matrix $\left[G(\boldsymbol{p}, \boldsymbol{r})+G^{\prime}(\boldsymbol{p}, \boldsymbol{r})\right]$ is negative definite for $\boldsymbol{p} \in \mathcal{D}$, then the equilibrium point $\boldsymbol{p}^{*}$ of $\mathcal{G}$ is unique.

Proof. This lemma is straightforward result from Theorems (2) and (6) in [48].

We can now introduce the result of the uniqueness.

Theorem 1. The game $\mathcal{G}=\left(\mathcal{N},\left\{\mathcal{P}_{n}\right\},\left\{u_{n}\right\}\right)$ has a unique equilibrium point.

Proof. According to lemma (2), all we need is to prove that the sum of the Jacobian and its transpose is a negative definite matrix. Therefore,

$$
G(\mathbf{p}, \mathbf{r})=\left[\begin{array}{cccc}
r_{1} \frac{-\alpha_{1} h_{11}^{2}}{d_{1}^{2}}-2 \lambda_{1} & r_{1} \frac{-\beta_{1} \alpha_{1} h_{11} h_{12}}{d_{1}^{2}} & \cdots & r_{1} \frac{-\beta_{1} \alpha_{1} h_{11} h_{1 N}}{d_{1}^{2}} \\
r_{2} \frac{-\beta_{2} \alpha_{2} h_{22} h_{21}}{d_{2}^{2}} & r_{2} \frac{-\alpha_{2} h_{22}^{2}}{d_{2}^{2}}-2 \lambda_{2} & \cdots & r_{2} \frac{-\beta_{2} \alpha_{2} h_{22} h_{2 N}}{d_{2}^{2}} \\
\vdots & \vdots & \ddots & \vdots \\
r_{N} \frac{-\beta_{N} \alpha_{N} h_{N N} h_{N 1}}{d_{N}^{2}} & \cdots & \cdots & r_{N} \frac{-\alpha_{N} h_{N N}^{2}}{d_{N}^{2}}-2 \lambda_{N}
\end{array}\right],
$$

where $d_{n}=\beta_{n}\left(I_{n}+\sigma_{n}^{2}\right)+p_{n} h_{n n}$.

Clearly, for every $\mathbf{p} \in \mathcal{D}, \mathbf{p}^{T}\left[G(\mathbf{p}, \mathbf{r})+G^{\prime}(\mathbf{p}, \mathbf{r})\right] \mathbf{p}<0$. Therefore, $[G(\mathbf{p}, \mathbf{r})+$ $\left.G^{\prime}(\mathbf{p}, \mathbf{r})\right]$ is a negative definite and the claim that game $\mathcal{G}$ has a unique equilibrium follows. 
We generalize the above result in the following deduced corollary, which we will not prove due to its obviousness:

Corollary 1. For any power control technique based on a non-cooperative game, which has a NE, and whose utility functions are $u_{n} \forall n \in \mathcal{N}$. The NE point of this game is unique if for all $n \in \mathcal{N}$ we have $\frac{\partial^{2} u_{n}}{\partial p_{n} \partial p_{k}}<0$ for all $k \in \mathcal{N}$.

\subsubsection{Game Equilibrium Outcome}

In this subsection, we analytically find the Nash equilibrium point of the game $\mathcal{G}=$ $\left(\mathcal{N},\left\{\mathcal{P}_{n}\right\},\left\{u_{n}\right\}\right)$. During this treatment we also characterize the pricing factor $\lambda_{n}$. The following theorem provides the outcome of the game:

Theorem 2. The equilibrium point of $\mathcal{G}$ is given by:

$$
\begin{array}{r}
p_{n}^{*}=\frac{\beta_{n} I n_{n}\left(\boldsymbol{p}_{-n}^{*}\right)}{2 h_{n n}}\left(\sqrt{1+\frac{2 \alpha_{n}}{\lambda_{n}}\left(\frac{h_{n n}}{\beta_{n} I n_{n}\left(\boldsymbol{p}_{-n}^{*}\right)}\right)^{2}}-1\right) \\
(n=1,2, \ldots, N),
\end{array}
$$

where

$$
\lambda_{n} \geq \frac{\alpha_{n}}{2 p_{n}^{\max }\left(\frac{\beta_{n} \sigma_{n}^{2}}{h_{n n}}+p_{n}^{\max }\right)},
$$

and $\operatorname{In}_{n}\left(\boldsymbol{p}_{-n}^{*}\right)$ is the interference power plus noise power experienced by SBS $n$.

Proof. Since the Nash equilibrium is the best response of each $S B S_{n}$ to the other players' actions, we will fix the other players' power levels $\mathbf{p}_{-n}$ and find the best response power level $p_{n}^{*}$ that maximizes utility value of $S B S_{n}$. Intuitively, the best response of $S B S_{n}$ is the point at which the derivative of $u_{n}$ with respect to $p_{n}$ is zero. Hence,

$$
\begin{gathered}
\frac{\partial u_{n}\left(p_{n}, \mathbf{p}_{-n}\right)}{\partial p_{n}}=0=\frac{\alpha_{n}}{1+\frac{\gamma_{n}}{\beta_{n}}} \cdot \frac{1}{\beta_{n}} \frac{h_{n n}}{I_{n}\left(\mathbf{p}_{-n}\right)+\sigma_{n}^{2}}-2 \lambda_{n} p_{n}^{*} \\
\Rightarrow \alpha_{n} h_{n n}=2 \lambda_{n} p_{n}^{*}\left[\beta_{n}\left(I_{n}\left(\mathbf{p}_{-n}\right)+\sigma_{n}^{2}\right)+p_{n}^{*} h_{n n}\right] .
\end{gathered}
$$


For convenience, let the interference plus noise power $\left(I_{n}\left(\mathbf{p}_{-n}\right)+\sigma_{n}^{2}\right)$ be $\operatorname{In}_{n}\left(\mathbf{p}_{-n}\right)$ or simply $I n_{n}$. Then,

$$
2 \lambda_{n} h_{n n}\left(p_{n}^{*}\right)^{2}+2 \lambda_{n} \beta_{n} I_{n} p_{n}^{*}-\alpha_{n} h_{n n}=0
$$

Solving for $p_{n}^{*}$ :

$$
p_{n}^{*}=\frac{-2 \lambda_{n} \beta_{n} I n_{n} \pm \sqrt{\left(2 \lambda_{n} \beta_{n} I n_{n}\right)^{2}+8 \lambda_{n} \alpha_{n} h_{n n}^{2}}}{4 \lambda_{n} h_{n n}} .
$$

Since $p_{n}^{*} \geq 0$, we will restrict ourselves on the positive values of $p_{n}^{*}$. Therefore,

$$
p_{n}^{*}=\frac{\beta_{n} I n_{n}\left(\mathbf{p}_{-n}\right)}{2 h_{n n}}\left(\sqrt{1+\frac{2 \alpha_{n}}{\lambda_{n}}\left(\frac{h_{n n}}{\beta_{n} I n_{n}\left(\mathbf{p}_{-n}\right)}\right)^{2}}-1\right)
$$

Since every player $n \in \mathcal{N}$ seeks to maximize its own utility, then each $S B S n$ would select its transmit power policy to be $p_{n}^{*}$ using (4.13). Accordingly,

$$
\begin{array}{r}
p_{n}^{*}=\frac{\beta_{n} I n_{n}\left(\mathbf{p}_{-n}^{*}\right)}{2 h_{n n}}\left(\sqrt{1+\frac{2 \alpha_{n}}{\lambda_{n}}\left(\frac{h_{n n}}{\beta_{n} I n_{n}\left(\mathbf{p}_{-n}^{*}\right)}\right)^{2}}-1\right), \\
(n=1,2, \ldots, N) .
\end{array}
$$

To make sure that $p_{n}^{*}$ is bounded by $p_{n}^{\max }$, we will impose a condition on $(4.14)$ such that the following inequality is maintained.

$$
p_{n}^{*} \leq p_{n}^{\max }, \forall n \in \mathcal{N}
$$

Using the value of $p_{n}^{*}$ in (4.14), the required condition in (4.15) becomes

$$
I_{n}\left(\mathbf{p}_{-n}^{*}\right) \geq-\frac{h_{n n}}{\beta_{n}}\left(p_{n}^{\max }-\frac{\alpha_{n}}{2 \lambda_{n} p_{n}^{\max }}\right)-\sigma_{n}^{2}, \forall n \in \mathcal{N}
$$

Since interference power is a positive value, (4.16) is always true, and hence inequality 
in (4.15) is always maintained as long as:

$$
\lambda_{n} \geq \frac{\alpha_{n}}{2 p_{n}^{\max }\left(\frac{\beta_{n} \sigma_{n}^{2}}{h_{n n}}+p_{n}^{\max }\right)}, \forall n \in \mathcal{N}
$$

This completes the proof.

Note that the selected value of $\lambda_{n}$ in (4.17) captures the property mentioned in Subsection 4.3.2 that requires the pricing factor to be location dependent. As per (4.17), it is clear that as the user goes farther from its serving SBS, as its channel becomes weaker. Hence $\lambda_{n}$ gets a lower value compared to the pricing factor of a SCU that is closer to its SBS.

As the value of $\lambda_{n}$ increases farther from the right hand side of (4.17), as the transmit power $\left(p_{n}^{*}\right)$ at equilibrium point decreases a way from $p_{n}^{\max }$. This would be the case even if the interference power is zero (i.e., $N=1$ ). So, this would adversely decrease the spectral efficiency as shown in Fig. 4.4(a) because intuitively if the interference power is zero, $S C U_{1}$ should transmit with full power. Thus, from spectral efficiency viewpoint, the best choice of $\lambda_{n}$ is such that when interference is

zero, the selected transmit power would be $p_{n}^{\max }$. This is equivalent to the case when $\lambda_{n}$ strictly equals the right hand side of (4.17). From engineering viewpoint, however, if all users increase their $\lambda_{n}$ by a certain factor, their loss in spectral efficiency is very seldom compared to the gain in their energy efficiency as shown in Fig. 4.4.

\subsubsection{Distributed Power Control Algorithm}

Nash equilibrium of the above game can be easily found in a centralized way if global system information is available. This could be done by rewriting (4.10) as:

$$
\frac{\partial u_{n}}{\partial p_{n}}=\frac{\alpha_{n} h_{n n}}{\beta_{n}\left(\sum_{k \neq n} p_{k} h_{n k}+\sigma_{n}^{2}\right)+h_{n n} p_{n}}-2 \lambda_{n} p_{n}=0
$$




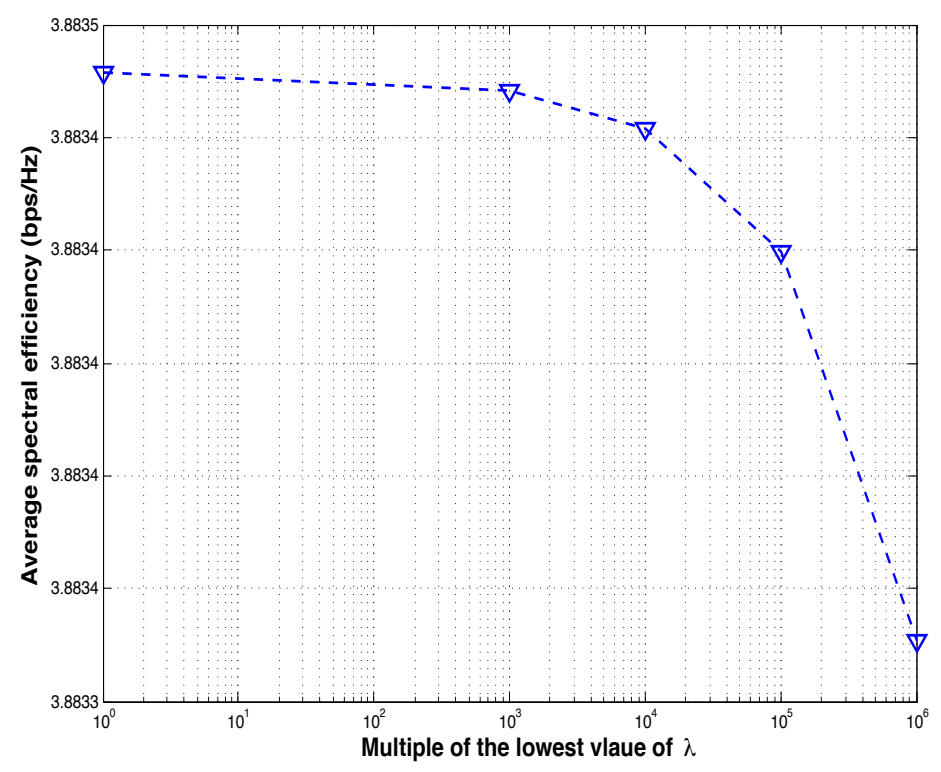

(a)

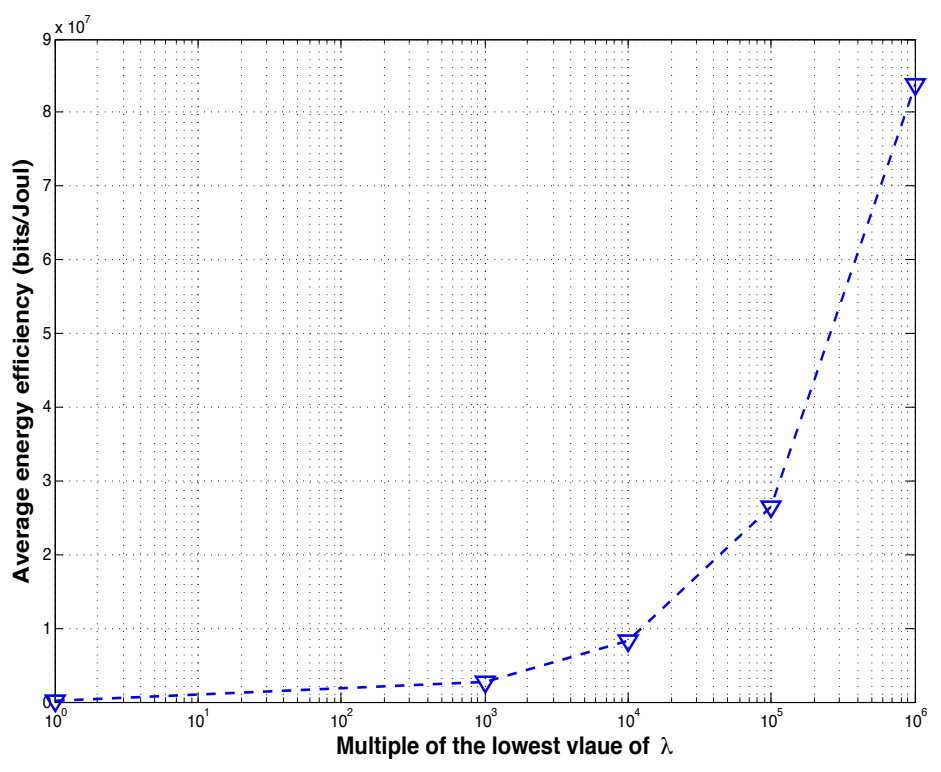

(b)

Figure 4.4: The impact of increasing $\lambda_{n}$ beyond the RHS of (4.17) on: a) spectral efficiency, and b) energy efficiency. The number of small cells in this example is 6 .

for all $n \in \mathcal{N}$. Since there are $N$ users, then there are $N$ equations with $N$ unknown variables (i.e., $p_{n} ; n=1,2, \ldots, N$.). However, this non-cooperative game approach is considered suboptimal in a centralized setup. Another disadvantage of the centralized solution is that all parameters (i.e., $h_{n n}, \beta_{n}, h_{k n}, \lambda_{n}$ for all $n$ ) of the above system are required to be sent to a central entity for computation. Due to the tremendous difficulty of gathering global information in heterogeneous networks, we propose the following fully distributed algorithm.

It is obvious that the Nash equilibrium point is $\mathbf{p}^{*}=\left(p_{1}^{*}, p_{2}^{*}, \ldots, p_{N}^{*}\right)$. If we write (4.9) in a vector form, then

$$
\mathbf{p}^{*}=\mathbf{F}\left(\mathbf{p}^{*}\right)
$$

where $\mathbf{F}$ is a vector valued function whose element $F_{n}$ is equal to the right hand side of (4.9) corresponding to the link in the $n^{\text {th }}$ small cell. By definition of fixed point, it is noticeable that the Nash equilibrium of $\mathcal{G}$ is actually the fixed point of the function 
$\mathbf{F}(\mathbf{p})$ in (4.19). Moreover, since the game $\mathcal{G}$ has a unique Nash equilibrium due to theorem 1), $\mathbf{F}(\mathbf{p})$ must have a unique fixed point. Then we can rewrite (4.19) in a form of iterative power control algorithm,

$$
\mathbf{p}(t+1)=\mathbf{F}(\mathbf{p}(t))
$$

In general $\gamma_{n}=\frac{p_{n} h_{n n}}{\operatorname{In}\left(\mathbf{p}_{-n}\right)}$. Thus, we can write each element of the vector equation in $(4.20)$ as,

$$
\begin{array}{r}
p_{n}(t+1)=\frac{\beta_{n} p_{n}(t)}{2 \gamma_{n}(t)}\left(\sqrt{1+\frac{2 \alpha_{n}}{\lambda_{n}}\left(\frac{\gamma_{n}(t)}{\beta_{n} p_{n}(t)}\right)^{2}}-1\right) \\
(n=1,2, \ldots, N) .
\end{array}
$$

Therefore, each $S B S$ iterates by plugging into (4.21) its current SINR and current transmit power until it reaches the fixed point (i.e., NE), where each user will keep maintaining it as no one will benefit from a unilateral change. $\mathbf{F}$ is a contraction mapping [49, Chapter 3], and hence (4.20) is convergent. Moreover, the fact that the background receiver noise is nonzero implies the positivity of $\mathbf{F}(\mathbf{p})$, that is,

$$
\frac{\beta_{n} I n_{n}\left(\mathbf{p}_{-n}^{*}\right)}{2 h_{n n}}\left(\sqrt{1+\frac{2 \alpha_{n}}{\lambda_{n}}\left(\frac{h_{n n}}{\beta_{n} I n_{n}\left(\mathbf{p}_{-n}^{*}\right)}\right)^{2}}-1\right)>0 ; \quad \forall n
$$

Accordingly, $0<\mathbf{F}_{n}(\mathbf{p}) \leq p_{\max }$ for all $n$ that implies $\mathbf{F}(\mathcal{D}) \subset \mathcal{D}$. Therefore, the iteration would converge to $\mathbf{p}^{*}$ due to the guaranteed existence and uniqueness of the fixed point $\mathbf{p}^{*}$ within $\mathcal{D}$.

\subsubsection{Subchannels' Maximum Power Allocation}

Assume the total power available to each $S C U_{n}$ is $p_{\text {total }}^{(F)}$. For distributing the total power over the spectral channels and finding $p_{n m}^{\max } \forall m \in \mathcal{M}^{S}$, we suggest the following two alternatives, which in turn create two forms of the game that differ in the actions 
space $\left\{\mathcal{P}_{n m}\right\}_{n=1}^{N}$ :

- $p_{n m}^{\text {max }}=\frac{p_{\text {total }}^{(F)}}{\left|\mathcal{M}^{S}\right|}$. This is uniformly equal power distribution which we will refer to as equally $\mathbf{p}^{\max }$. Therefore,

$$
\left\{\mathcal{P}_{n m}^{e q}\right\}_{n=1}^{N}=\left\{\left[0, \frac{p_{\text {total }}^{(F)}}{\left|\mathcal{M}^{S}\right|}\right]\right\}_{n=1}^{N}, \forall m \in \mathcal{M}^{S}
$$

- $p_{n m}^{\max }=\frac{h_{n m}}{\sum_{m \in \mathcal{M}^{S} h_{n m}}} p_{\text {total }}^{(F)}$. This distribution is proportional to the relative strength of the channel $m$. In other words, the stronger the subchannel is, the more its power space would be. Hence, we will refer to this distribution as proportional $\mathrm{p}^{\max }$; thus,

$$
\begin{aligned}
\left\{\mathcal{P}_{n m}^{\text {prop. }}\right\}_{n=1}^{N}= & \left\{\left[0, \frac{h_{n m}}{\sum_{m \in \mathcal{M}^{S}} h_{n m}} p_{\text {total }}^{(F)}\right]\right\}_{n=1}^{N}, \\
& \forall m \in \mathcal{M}^{S}
\end{aligned}
$$

Since $p_{n m}^{*} \leq p_{n m}^{\max } \forall m \in \mathcal{M}^{S}$, we have $\sum_{m \in \mathcal{M}^{S}} p_{n m}^{*} \leq p_{\text {total }}^{(F)}$. In both forms, therefore, the total power constraint is always maintained.

\subsection{Simulation Results and Discussions}

In this section, the effectiveness of the proposed resource allocation scheme and the game-based interference management is shown through computer simulations. Under the system model mentioned in Section 4.1 and simulation parameters shown in Table 4.1, we simulated a macrocell area consisting of a $M B S$ associated with a number of $M U s$, and several $S B S s$ associated with their corresponding $S C U s$. For small cells, we tested two topologies. In the first simulation, small cells are deployed in an organized topology (e.g., Hexagonal). This topology reflects the case of picocells or the case of smaller cells under the authority of one enterprise, where in both cases the 
locations of small BSs are carefully selected. In the second simulation, small cells are assumed to be randomly deployed by the end users, reflecting the case of femtocells in a residential area. In the latter case, the locations of SBSs are picked according to the Poisson point process (PPP). Moreover, to ensure that sufficient data is collected, we first replicated the simulation for 40 runs. Then, we increased them to 60 runs, but we didn't find in the results significant differences. Therefore, we are sufficed by 60 runs. A general simulation setup is described in Appendix A.

We compare our proposed approach with an existing interference control scheme [50], which is also based on a game theoretic approach between all BSs including MBS. The channel assignment among MUs is performed using Hungarian algorithm on the matrix of MUs' desired channels. Then, the game is to be played over each subchannel by all BSs. The action of each player is the transmit power. If $m^{\text {th }}$ subchannel was assigned to $j^{\text {th }} \mathrm{MU}$, the utility function of MBS is $-\left(\gamma_{j m}-\Gamma_{j m}\right)^{2}$, where $\gamma_{j m}(t)=\frac{p_{j m}(t) h_{0 j m}(t)}{\sum_{k \in \mathcal{N}} p_{k m}(t) h_{n k m}(t)+\sigma_{0 m}^{2}}$, and $\Gamma_{j m}$ is the target SINR required by $M U_{j}$ over $m^{\text {th }}$ subchannel. On the other hand, the utility function of each SBS is $u_{n m}=\alpha_{n} \ln \left(1+\frac{\gamma_{n m}}{\beta_{n m}}\right)-\lambda_{n m} p_{n m}$, where the penalty part here is linear on the transmit power [50]. Since this game includes both MBS and SBSs to jointly control both cross-layer and co-layer interference, we refer to this existing scheme by Joint Game. The convergence criterion in the proposed schemes as well as the Joint Game scheme is $\left|p_{n}(t+1)-p_{n}(t)\right| \leq 0.5 \times 10^{-7}$ for all players.

Furthermore, frequency coordination among adjacent base stations is a well-known approach for interference management [22], [7, Chapter 3]. In the organized deployment, since coordinating the spectrum usage among the adjacent base stations is doable, we also compare the proposed game-based algorithm with two schemes: frequency reuse-3 (referred to as Reuse-3) and soft frequency reuse (SFR). Reuse-3 is known to be very effective in alleviating intercell interference on border users. However, that comes out of a weakened aggregate spectral efficiency. SFR was proposed 
essentially to improve the aggregate spectral efficiency of Reuse-3. However, the border users in SFR would experience relatively higher interference than in Reuse-3.

When the deployment is random, fixed spectrum coordination between adjacent cells is inherently difficult due to the random locations of SBSs. In addition, intercell coordination for spectrum access in SBSs owned by end users is usually prohibitive. Therefore, only the Joint Game scheme is compared with the proposed game-based schemes.

According to the parameters in Table 4.1, it has been found that when algorithm 1 is applied in the MBS, 6 MUs can be satisfied by assigning them on average 23.647 subchannels, assuming an adaptive modulation and coding scheme with high levels is used. Specifically, the $95 \%$ confidence interval of the number of subchannels occupied by 6 MUs is $[23.0313,24.2627]$. Thus the average resource consumption of MUs is only $8.27 \%$ of the system radio resources. Fig. 4.5 shows the linear relationship between the number of MUs and the number of their occupied subchannels. The linearity relationship is due to the fact that Hungarian algorithm is an efficient algorithm to integer linear programming beside also having uniform required rate. Furthermore, since energy saving policy is applied in MBS algorithm, it has been found that the $95 \%$ confidence interval of the energy efficiency is $[1.6427,2.8108] \mathrm{Mb} / \mathrm{J}$. The following two subsections test the effectiveness of the game-based interference control among small cells.

\subsubsection{Organized Deployment}

In a hexagonal topology, we tested the proposed scheme, which appears in two forms (equally $p^{\max }$ and proportional $p^{\max }$ ), the Joint Game scheme, SFR and Reuse-3. Unlike the game-based schemes whose SBSs have full spectrum access, the granted spectrum in Reuse-3 is divided into 3 sub-bands. Each SBS in Reuse-3 is assigned a sub-band, which is orthogonal to the allocated sub-bands in the adjacent SBSs. In 
Table 3: Simulation Parameters

\begin{tabular}{|c|c|}
\hline Parameters & Value \\
\hline \hline System frequency & $2 \mathrm{GHz}$ \\
\hline System bandwidth & $5 \mathrm{MHz}$ \\
\hline No. of data subchannels & 300 \\
\hline Subchannel bandwidth & $15 \mathrm{KHz}$ \\
\hline Small cell radius & $30 \mathrm{~m}$ \\
\hline$p_{\text {total }}^{(C)} p_{\text {total }}^{(F)}$ & $33,30 \mathrm{dBm}($ respectively) \\
\hline MU required rate: $R_{j} \forall j \in \mathcal{J}$ & $1 \mathrm{Mbps}$ \\
\hline Noise power spectral density & $-174 \mathrm{dBm} / \mathrm{Hz}$ \\
\hline Shadow fading deviation & $8 \mathrm{~dB}$ \\
\hline Small scale fading & Rayleigh fading \\
\hline Target BER & $10 \mathrm{e}-6$ \\
\hline Number of time slots & 100 \\
\hline Number of replications & 60 \\
\hline Link & Uplink \\
\hline
\end{tabular}

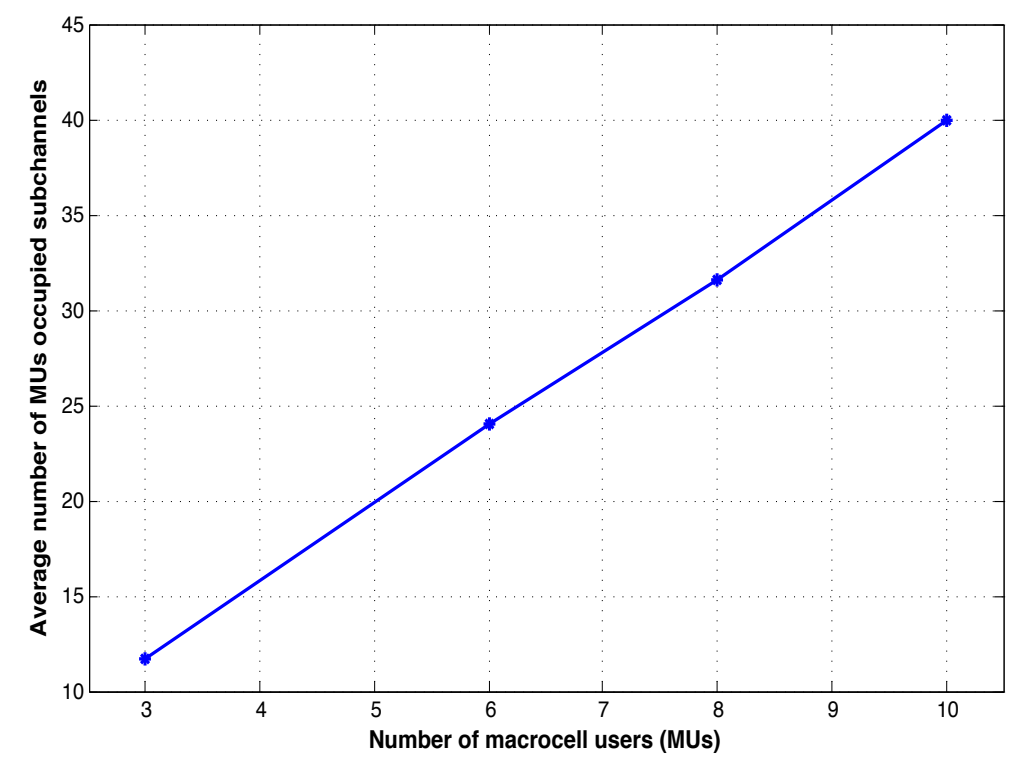

Figure 4.5: Relationship between the number of macrocell users and the number of their assigned subchannels using Algorithm 1. 
SFR, the central users can access the whole granted spectrum whereas the border user can only access one of three equal portions of the spectrum. These three portions are orthogonal on adjacent cells. SCUs in Reuse-3 and border users in SFR divide the total transmit power equally over the assigned subchannels. In SFR, the ratio of the central users transmit powers to the border users transmit powers is $1 / 4$ [22].

Fig. 4.6 shows that while the spectral efficiency of both game-based schemes (the two proposed forms and Joint Game) is almost the same, the spectral efficiency of both outperform that of Reuse- 3 and SFR by about $51 \%$ and $21 \%$, respectively ${ }^{2}$. In general, the game-based scheme outperforms both Reuse-3 and SFR in every test performance metric. This is due to the following reasons:

- The game-based schemes take into account not only the desired channel gain but also the amount of experienced interference.

- These schemes impose a cost over power spending. Hence, they force each transmitter to spend responsibly.

- The price factor $\lambda_{n}$ is a non-uniform location-dependent.

In terms of border user efficiency, it is well known that Reuse-3 is very effective in serving border users; however, Fig. 4.7 shows that Reuse-3 is outperformed by the game-based schemes by at least $25 \%$. In addition to the above mentioned reasons, this enhancement is because the game is played with all interferers in the network, unlike the case of Reuse-3 where coordination is performed only among the adjacent cells. Furthermore, the number of spectral subchannels available to the game based scheme is 3 times of that in Reuse-3.

From Fig. 4.7, it is noticeable that the proposed schemes is slightly more efficient in serving the border user than the Joint Game by on average 4\%. This can be

\footnotetext{
${ }^{2}$ This percentage is the average of improvement percentages across the reading points in the mentioned graph.
} 
justified as follows. Since $\lambda$ is location dependent, the border user is allowed to spend relatively high power. Furthermore, the penalty part in the proposed scheme is quadratic in transmit power, which would force the central users to carefully use their power. For that reason, the energy efficiency (in Fig. 4.8) of the proposed scheme also outperforms that of the Joint Game scheme by at least $25 \%$.

The decreasing trend in all schemes and all efficiency metrics mentioned above is due to the fact that the amount of the interference power increases as the network is densified. The increase in the interference would cause a reduction in the SINR at each receiving node.

Fig. 4.9 shows the cumulative density function (CDF) of the number of iterations before the game-based schemes converge to the NE point. When the number of SBSs is 27, Our proposed scheme requires on average about 6 iterations while the Joint Game scheme requires 32 iterations. Moreover, the variance of the number of required iterations in the proposed scheme is much less than that of the Joint Game scheme $(0.9505<2335)$. Thus, the convergence time in the proposed scheme is more stable and predictable.

Finally, it is noticeable that the equally $p^{\max }$ form performs slightly better than the proportional $p^{\max }$ form. That is because the action set in the equally $p^{\max }$ form is known to each players, rather than that in the proportional $p^{\max }$ form which depends on the desired channels of every players which is unknown to the other players.

\subsubsection{Spontaneous Deployment}

This topology represents the case of small cells in a residential region where SBSs are installed in an ad hoc fashion by the end users. While the average density of SBSs is identical to that in the previous subsection, the simulator randomly selects the locations of the SBSs according to the Poisson point process (PPP). The proposed scheme is compared here against a system equipped with the Joint Game scheme. 


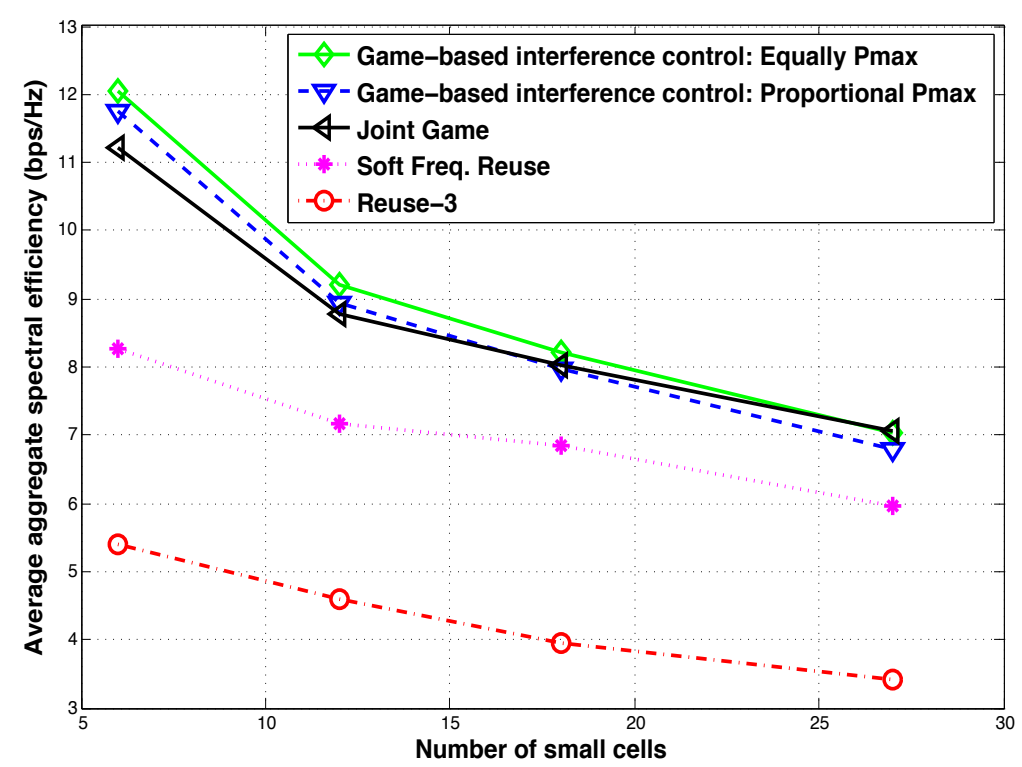

Figure 4.6: Average aggregate spectral efficiency in the organized network topology.

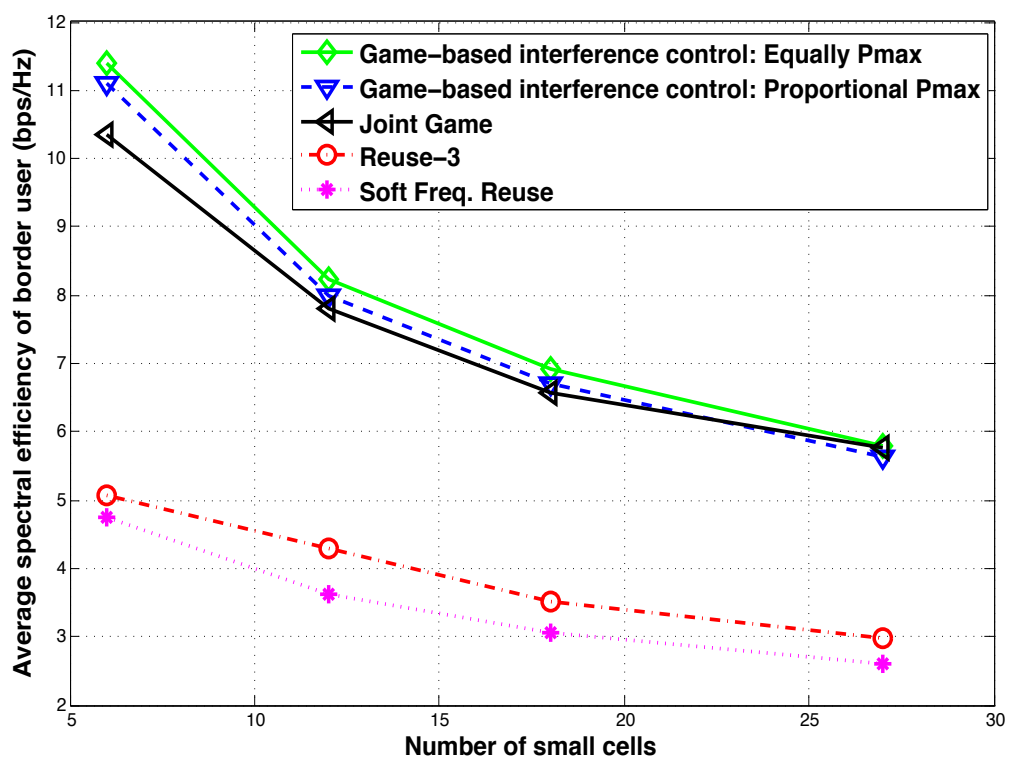

Figure 4.7: Average border user spectral efficiency in the organized network topology. 


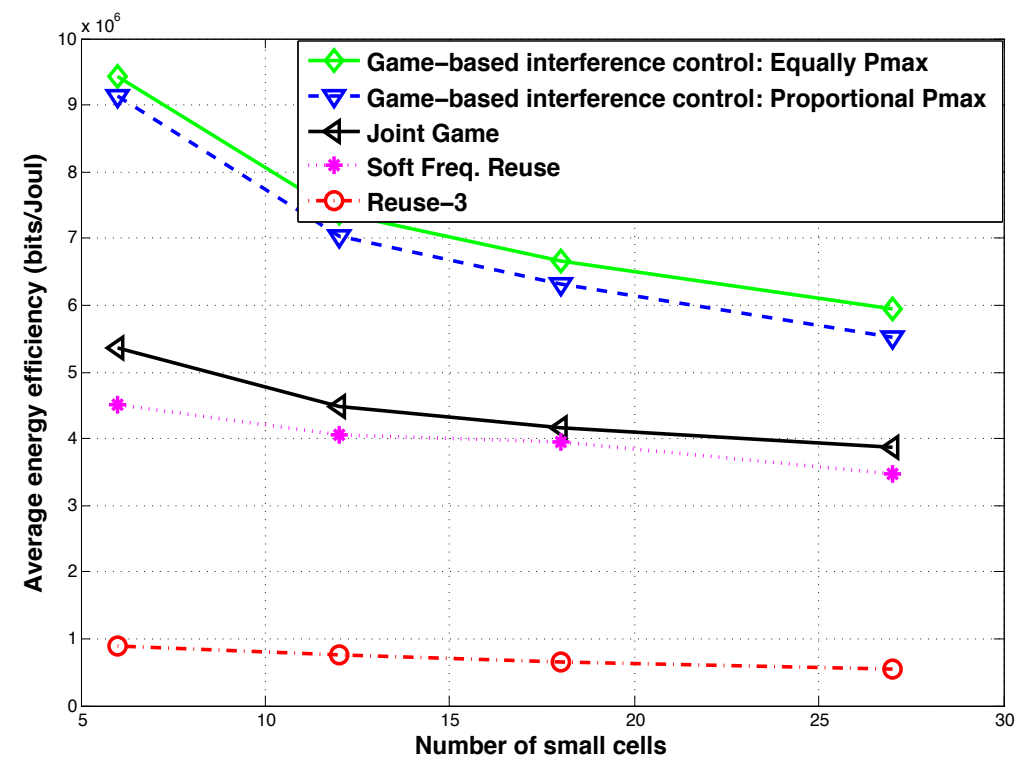

Figure 4.8: Average energy efficiency in the organized network topology.

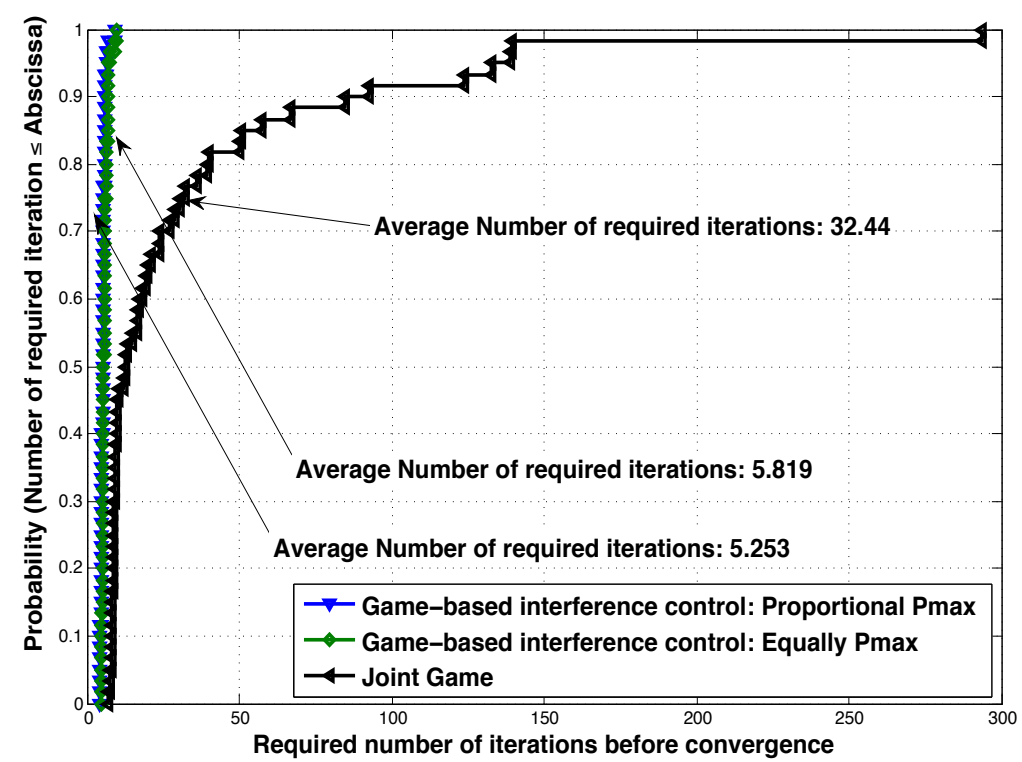

Figure 4.9: Empirical CDF of the required iterations for the game-based schemes to converge (The number of SBSs is 27). 


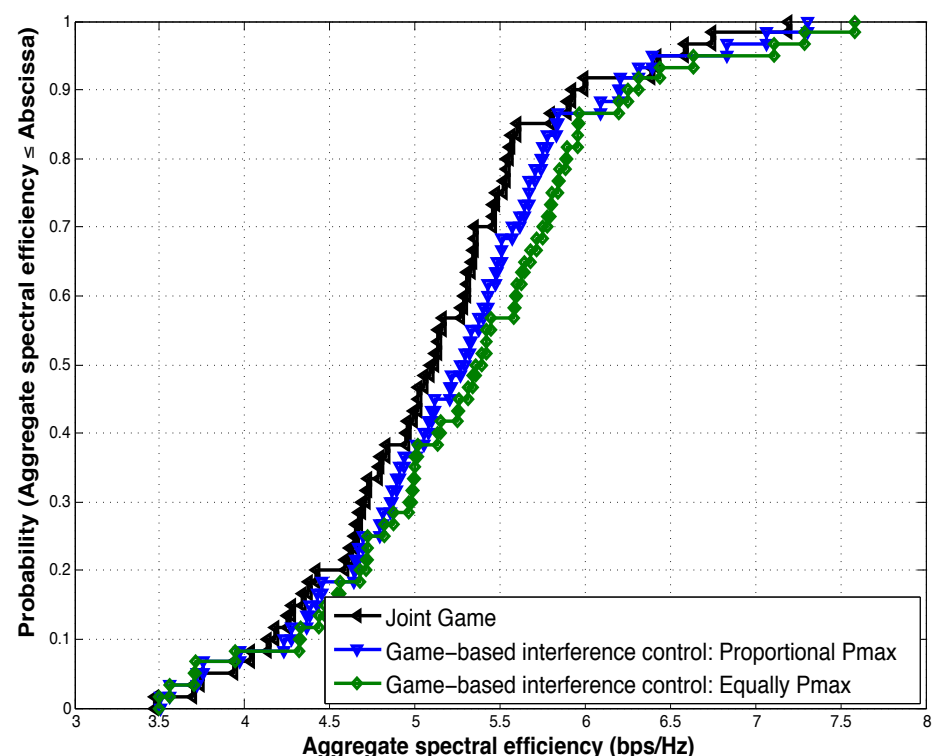

(a)

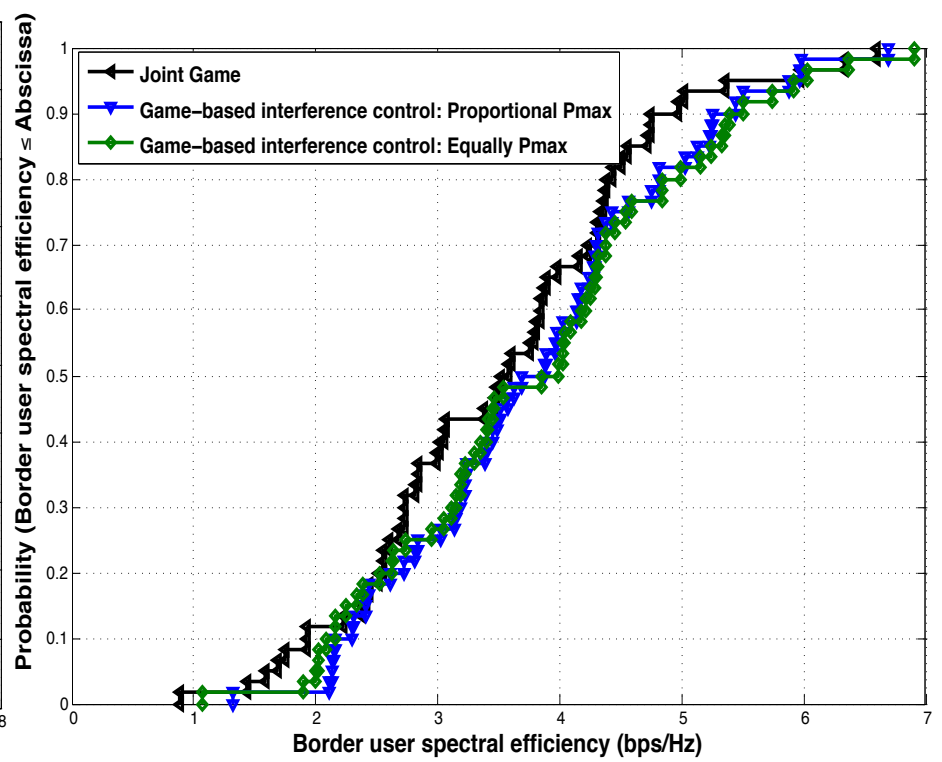

(b)

Figure 4.10: Empirical CDF of the a) aggregate spectral efficiency, and b) border user spectral efficiency (random topology).

Fig. 4.10 shows cumulative density function of the aggregate spectral efficiency and border user spectral efficiency when the average number of small cells is 27 . In both metric, the two forms of our proposed scheme outperform the Joint Game scheme. This is due to the tightly controlled interference by the proposed game-based schemes. Again, responsible energy spending due to the quadratic-based penalty, and the location-dependent price factor all play an effective role in controlling the interference.

Fig. 4.11 shows the energy efficiency with respect to the average number of small cells. The proposed scheme shows a significant higher performance. The energy efficiency of the equally $p^{\max }$ form and the proportional $p^{\max }$ form outperform that of Joint Game by about $66 \%$ and $53 \%$, respectively.

Fig. 4.12 shows that as the number of players increases, the number of required iterations before convergence increases as well. This is because the period of contradicting each other until they all settle to NE will prolong as the number of players 


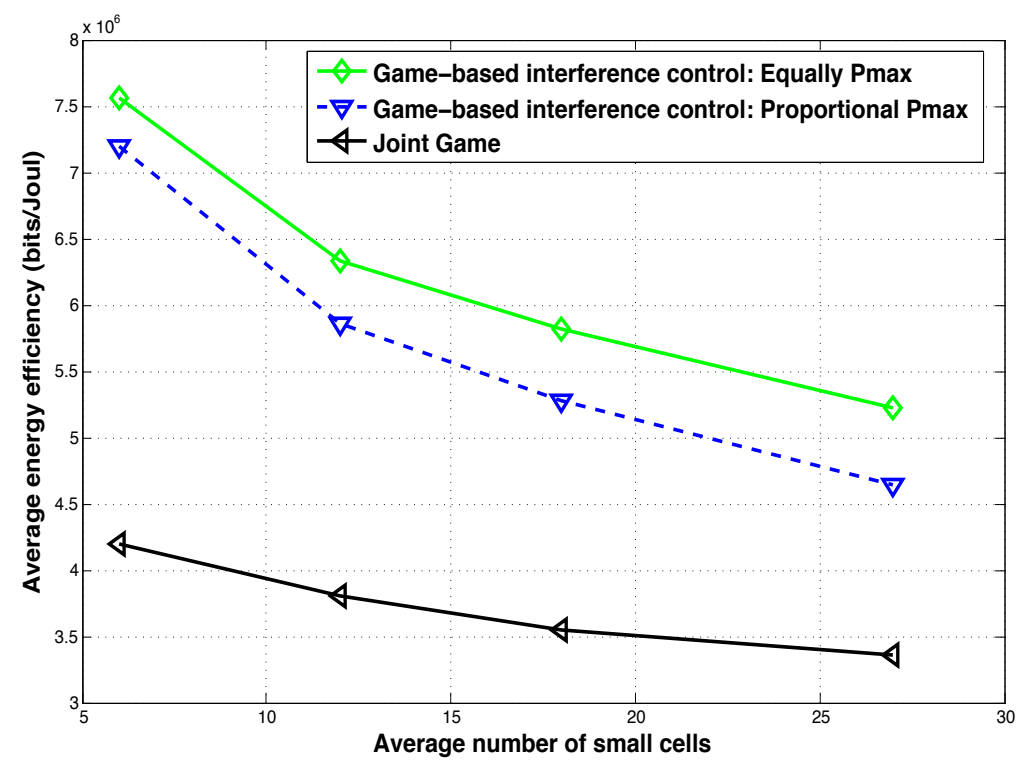

Figure 4.11: Average energy efficiency in a random topology.

increases. At a given iteration, the probability of having a player, who have not yet reached to NE, in a 50-player game is higher than the probability of having this unsettled player in a 5-player game.

Finally, a noteworthy advantage of carefully choosing the locations of SBSs appears when the spectral efficiency of the game-based forms in Fig. 4.6 and Fig. 4.13 are compared. Clearly, the performance of the game-based solution in the organized topology is better than that of the random topology by about $10 \%$.

\subsection{Conclusions}

In this chapter, a novel energy-efficient resource allocation and interference control scheme using the game theoretic approach has been proposed for green heterogeneous networks. Since macrocell users may have higher priority from operators' viewpoint, their QoS requirements shall be met. However, MBS operation is not as efficient as that of small cells in terms of spectral efficiency and energy efficiency. Therefore, we proposed a near-optimal resource allocation algorithm in the macrocell level that 


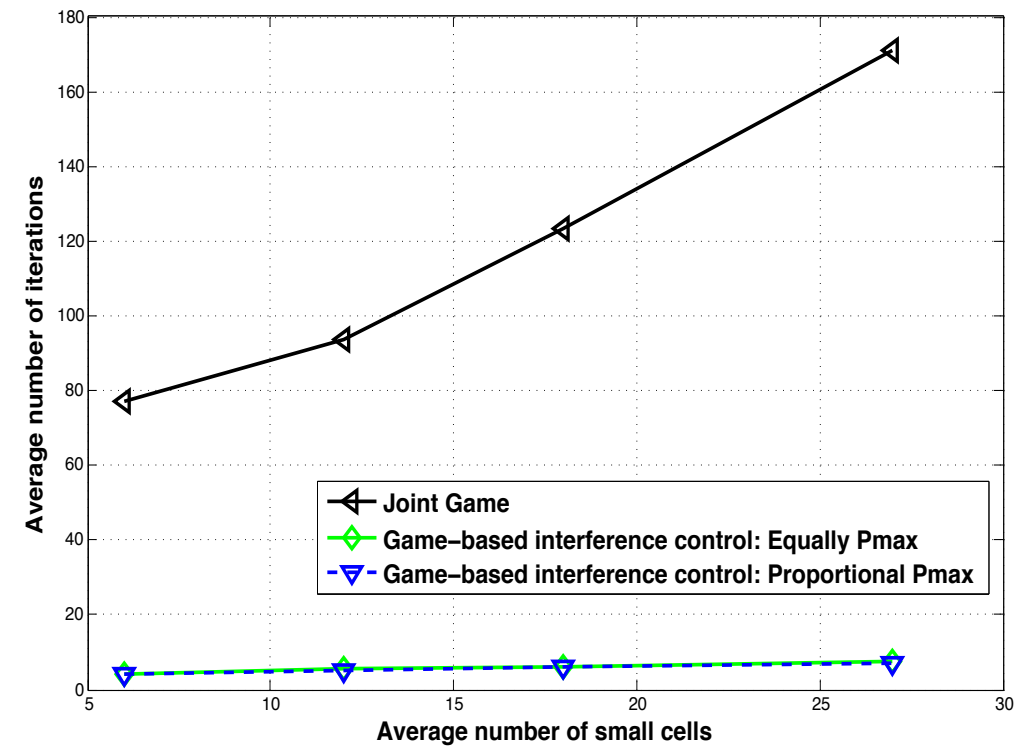

Figure 4.12: Average number of iterations to convergence (random topology).

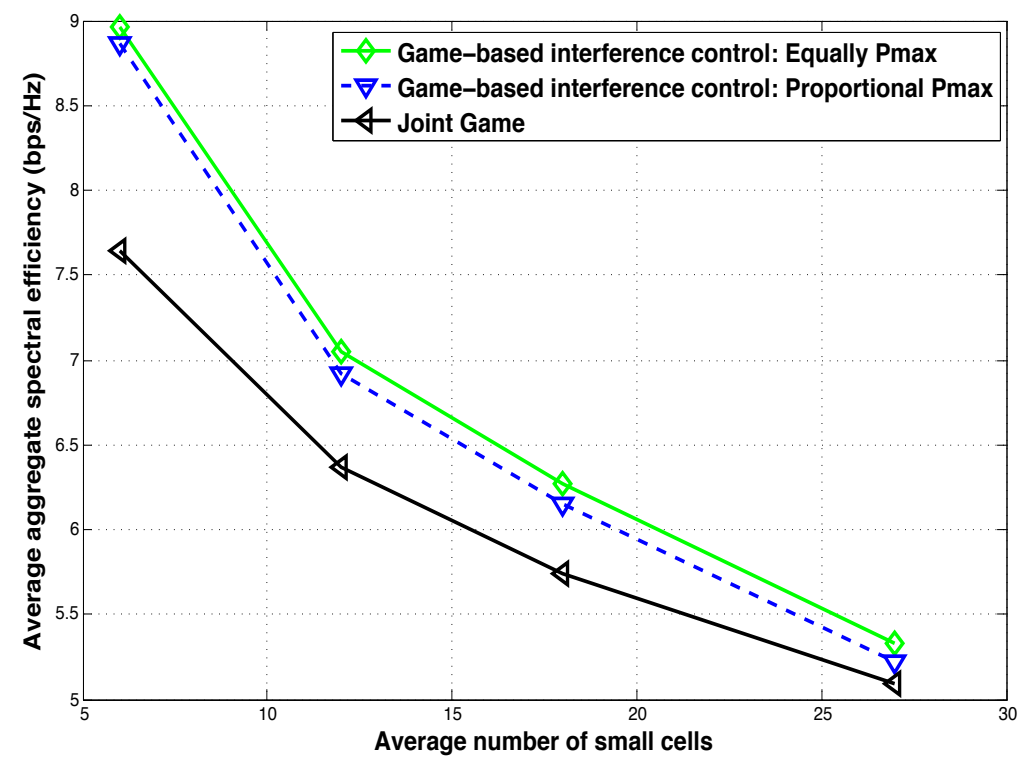

Figure 4.13: Average aggregate spectral efficiency in the random topology. 
assigns minimal required resources to the macrocell users. The remaining bulk of subcahnnels are then shared by all small cells using a game-based scheme. Using economic concepts, the utility function of every user has been designed to include two parts, a reward part and a cost part. The utility function, which captures both fairness and bit error rate requirement, makes sure that the level of service received by each user is worth exactly the price it pays. Furthermore, the pricing factor is fully characterized such that maximum power constraint are maintained. The action of every co-channel small cell user is to transmit in an optimal power level, seeking to maximize its own utility. Such strategy leads to a justified interference in the air. Game analysis demonstrated that the proposed non-cooperative game has a unique Nash equilibrium point. In two different network topologies, simulation results has shown that the game-based interference control scheme outperforms the game based scheme with linear penalty part, Reuse-3, and soft frequency reuse schemes. These improvements are not only in terms of the aggregate spectral efficiency and energy efficiency, but also includes significant enhancement in the efficiency of the border users. 


\section{Chapter 5}

\section{Resource Allocation and Interference Management in HetNets with a Large Number of Small Cells}

In the previous chapter, we considered HetNet systems with a moderate number of small cells. In this chapter, we propose an inter-cell interference control scheme for HetNet systems that include a large number of small cells located within the macro-

cell area. In this scheme, two consecutive optimal algorithms are applied. First, an optimal algorithm, which runs at the MBS, is designed to ensure both MU QoS provision and sum rate maximization. Although the formulated optimization problem is mixed integer non-linear programming (MINLP), it has an interesting structure that we exploit to achieve the optimality. This algorithm ensures optimality by successively solving a convex optimization problem. The second algorithm is proposed to control the co-layer interference using an effective and promising solution, namely, the mean-field game theory (MFG). Due to the large number of interacting small cells, we exploit the system statistics to collect brief information about the system. Then, we formulate and solve a decentralized non-cooperative game. 
As mentioned in Chapter 2, the purpose of the MFG is to take decisions in a decentralized manner, with minimal computational effort, and using only local and minimal information. Unlike the decision making technique based on conventional game theory, which requires each player to collect each piece of information $[11-13,16,68]$, the MFG requires much less information. Nevertheless, the MFG provides an equilibrium solution as the number of players increases considerably. Moreover, the computations required to solve the MFG are much less complex [12-14].

The authors of [69] applied the mean-field game theory in a simple network of one receiver and several transmitters to design a green power control. They showed that when the number of transmitters is large, the stochastic differential game converges to a mean-field game that is ruled by a tractable system of equations. However, unlike the practical systems that work on discretized time, their analysis is based on a continuous time system.

In addition to the enhancement in spectral efficiency achieved by our solution, it substantially reduces the complexity of resource allocation. This merit of the MFGbased approach stems from a practical consideration. Obviously, most of the current interference control techniques assume that the channel information between interferers and a desired receiver is always available. However, estimating the interference channel from each and every interferer is very difficult and time consuming, let alone tracking them over time. In this research, we reduced this complex problem into one estimation requirement, which provides a brief picture of the system.

The rest of this chapter is organized as follows. The system model is described in Section 5.1. In Section 5.2, the optimization problem in macrocells is formulated and solved. Section 5.3 presents the mean-field approximation approach with a decentralized solution. Then, simulation results in two different topologies are introduced in Section 5.4. Finally, the chapter concludes with Section 5.5. 


\subsection{System Description}

We consider a discretized time, orthogonal frequency division multiplexing (OFDM) HetNet system. This system is comprised of $N$ SBS receivers and their associated user equipment (UE) transmitters, as well as one MBS receiver and its associated UE transmitter. The set of available radio subchannels is $\mathcal{M}=\{1, \ldots, m, \ldots, M\}$. Similar to [2], we assume one transmitting user per cell per time slot $^{1}$. Hence, the set of all cells' transmitter-receiver links is $\mathcal{N}=\{0,1,2, \ldots, N\}$, where 0 is the index of the macrocell transmitter-receiver link. Considering uplink, the signal received at base station $n \in \mathcal{N}$ is:

$$
y_{n}(t)=\sum_{k=0}^{N} \sqrt{h_{n k}(t)} * a_{k}(t)+z_{n}(t)
$$

where $\sqrt{h_{n k}(t)} \in \mathbb{C}$ is the channel coefficient between the transmitter associated to BS $k$ and the $n^{\text {th }}$ BS at time slot t. It is assumed that all the channels involved are independent and identically distributed (i.i.d). Since the OFDM transmission scheme is used, we assume each channel to be flat over each time slot (i.e., blockfading channels are considered). In the case of an abuse of notation, we will use the shorthand $h_{n}(t)=h_{n n}(t)$ to refer to the channel between $n^{\text {th }}$ UE and its serving BS. $a_{k}(t)$ is the sequence ${ }^{2}$ of the OFDM symbols sent by the transmitter $k$ associated with BS $k$ during the time slot t. $z_{n}(t)$ is a Gaussian noise with variance $\sigma^{2}$. The power transmitted by a transmitter $k$ at time slot $t$ is denoted by $p_{k}(t)=\left|a_{k}(t)\right|^{2}$.

The signal to interference plus noise ratio (SINR) at BS $n$ over subchannel $m$ at

\footnotetext{
${ }^{1}$ Note that, our work can be easily extended to several users per cell by applying an adaptive and dynamic scheduler such as the proportional fair scheduler.

${ }^{2}$ In the LTE uplink physical layer design, each slot is comprised of 7 OFDM symbols for the normal Cyclic Prefix (CP) length.
} 
time slot $t$ is ${ }^{3}$ :

$$
\gamma_{n m}(t)=\frac{p_{n m}(t)\left|h_{n m}(t)\right|}{\sum_{k \neq n}^{N} p_{k m}(t)\left|h_{n k m}(t)\right|+\sigma_{n m}^{2}} .
$$

Finally, we assume that BSs make decisions and dictate those decisions on their UEs.

\subsection{Formulation of the Macro-BS Optimization Problem}

To simultaneously ensure macrocell user satisfaction as well as a maximum network sum rate, it is reasonable to exclusively assign the macrocell user to the minimum number of subchannels, which fairly guarantees the required quality of service (QoS). The remaining bulk of subchannels are left for small cells, in order to maximize the network sum rate. Therefore, we propose the following optimization problem, whose solution is to be found by the macro BS at the beginning of every scheduling cycle:

$$
\begin{array}{ll}
\underset{\substack{X_{0 m}, \forall m \\
p_{0 m}, \forall m}}{\operatorname{minimize}} & \sum_{m \in \mathcal{M}} p_{\text {total }}^{(C)} X_{0 m}+p_{0 m} \\
\text { subject to } & \sum_{m \in \mathcal{M}} B \log _{2}\left(1+\frac{p_{0 m}\left|h_{0 m}\right|}{\beta_{0} \sigma_{0 m}^{2}}\right) X_{0 m} \geq R_{0} \text { (QoS const.) } \\
& \sum_{m \in \mathcal{M}} p_{0 m} \leq p_{\text {total }}^{(C)} \text { (Total Power Const.) } \\
& X_{0 m} \in\{0,1\}, \forall m \text { (Variable space) } \\
& p_{0 m} \geq 0, \forall m \text { (Nonnegativity), }
\end{array}
$$

where $X_{0 m}$ is a binary variable whose value is 1 when the $m^{\text {th }}$ subchannel is assigned to the cellular user, and 0 otherwise. $B$ is the subchannel bandwidth, $R_{0}$ is the cellular users required data rate, and $p_{\text {total }}^{(C)}$ is the cellular users total allowable transmit power. Moreover, $\beta_{0}=\frac{-\ln \left(5 B E R_{0}\right)}{1.5}$ is a positive factor that depends on the required bit error rate (BER). Since MBS is naturally less energy efficient than SBSs, the objective

\footnotetext{
${ }^{3}$ For ease of presentation, the time index $[\mathrm{t}]$ will be omitted in the subsequent.
} 
function is designed to support green policy in MBS. However, the solution of (5.3) focuses on minimizing the number of radio subchannels more than on minimizing the power consumption ${ }^{4}$.

This is a mixed integer non-linear programming (MINLP) problem that is analytically difficult. Fortunately, the above problem has special characteristics. Particularly, to guarantee the QoS of the MU via minimum resources, the MU should be assigned to subchannels whose power gains are the highest. Therefore, we propose Algorithm 2, which finds the optimal solution of (5.3) by successively solving a closely related convex optimization problem using the simple Kuhn-Tucker (KKT) technique. Note that in case all subchannels have been assigned to the MU before its QoS has been met, we resort to the best effort service using (5.5).

\subsection{Formulation of the Mean-Field Game among Small BSs}

In this section, we consider that each $\operatorname{SBS} n \in \mathcal{N} \backslash\{0\}$ is a rational player that competes with the other SBSs over the remaining subchannels (i.e. $\left.\mathcal{M}^{S}\right)^{5}$. Each SBS tries to decide the optimum power level on each subchannel. For every radio subchannel $m \in \mathcal{M}^{S}$, we will assume that each SBS is able to track the desired channel gain $\left|h_{n m}\right|$. Due to (5.2), all players are coupled via the interference term. Obviously, the variation of the interference experienced by SBS $n$ depends on two dynamic and independent vector variables: interferers' channel gains $\mathbf{h}_{-n m}=\left[h_{n 1 m}, \ldots, h_{n(n-1) m}, h_{n(n+1) m}, \ldots, h_{n N m}\right]^{T}$, and the vector of interferers transmit power $\mathbf{p}_{-n m}=\left[p_{1 m}, \ldots p_{(n-1) m}, p_{(n+1) m}, \ldots, p_{N m}\right]^{T}$. It is tremendously hard for each

\footnotetext{
${ }^{4}$ Since the first term in the objective function is multiplied by an expensive factor.

${ }^{5}$ In this section, each player $n$ is meant to be a SBS that belongs to $\mathcal{N} \backslash\{0\}$. Moreover, each subchannel $m$ is meant to be a remaining radio resource that belongs to $\mathcal{M}^{S}$, and is assigned to be used by SBSs.
} 


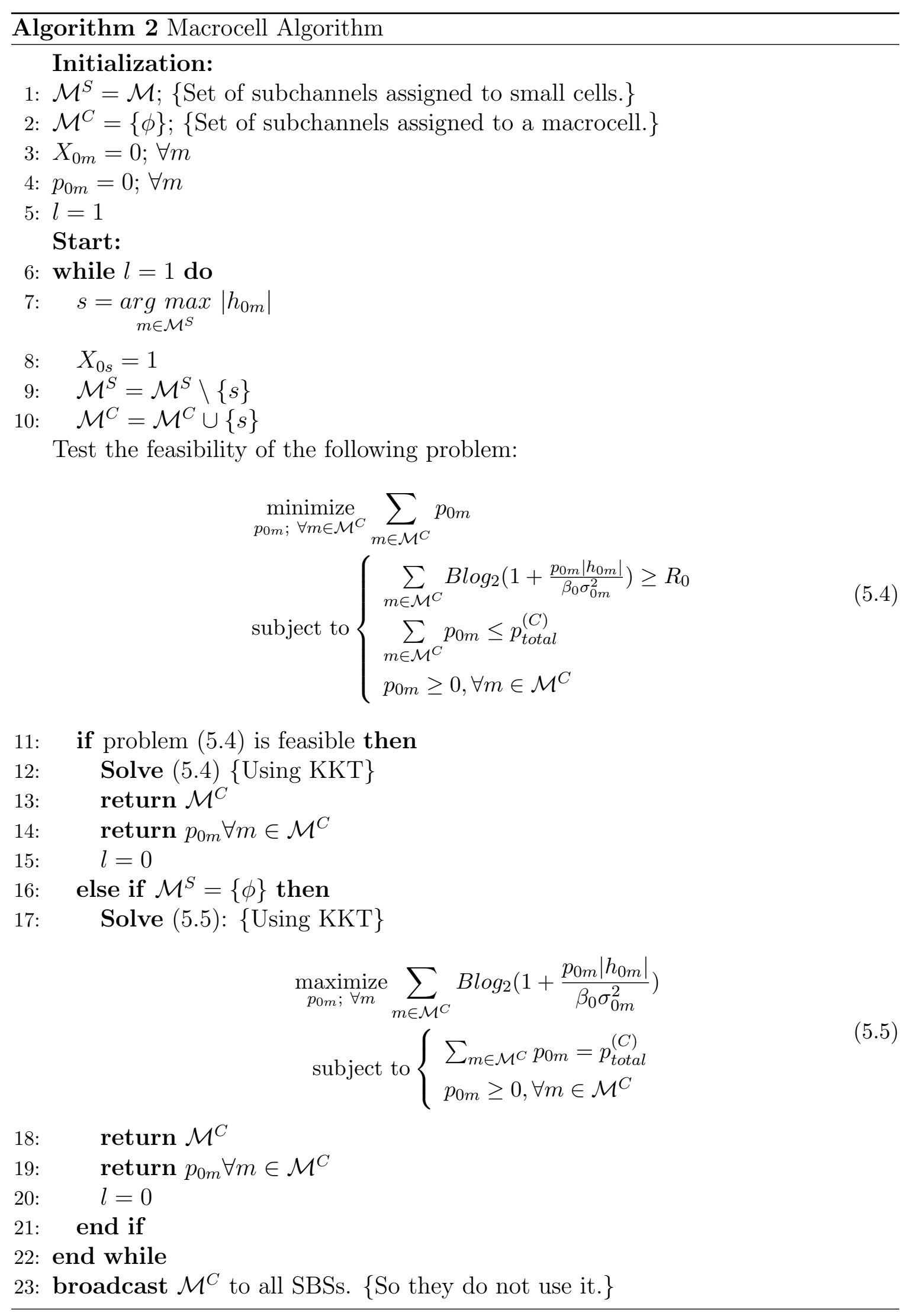


SBS $n$ to track $\mathbf{h}_{-n m}$ or to know in advance $\mathbf{p}_{-n m}$. However, if the number of interferers is quite large, a simplification of the problem can be accomplished by exploiting the knowledge of the statistics of these two vectors. The main concept is that, when there is a large number of players, the sample mean will converge to the statistical mean.

For every subchannel $m$, the interference at SBSs is $\mathbf{I}_{m}=\mathbf{H i}_{m} \cdot \mathbf{p}_{m}$, where $\mathbf{H i}_{m}$ is a zero-diagonal $N X N$ matrix whose off-diagonal elements $\left|h_{n k}\right|$ are interference channel gains from SCU associated with SBS $k$ to the SBS $n$,

$$
\left[\begin{array}{c}
I_{1 m} \\
I_{2 m} \\
\vdots \\
\\
I_{N m}
\end{array}\right]=\begin{array}{ccccc}
S B S_{1} \\
S B S_{2} \\
\vdots \\
S B S_{N}
\end{array}\left(\begin{array}{cccc}
0 & \left|h_{1,2}\right| & \cdots & \left|h_{1, N}\right| \\
\left|h_{2,1}\right| & 0 & \cdots & \left|h_{2, N}\right| \\
\vdots & \vdots & \ddots & \vdots \\
\left|h_{N, 1}\right| & \left|h_{N, 2}\right| & \cdots & 0
\end{array}\right)\left[\begin{array}{c}
p_{1 m} \\
p_{2 m} \\
\vdots \\
p_{N m}
\end{array}\right]
$$

In other words, the $n^{\text {th }}$ row in $\mathbf{H i}$ represents the vector of the independent channels' gains of the interfering signals coming to $\operatorname{SBS} n$. Since $N$ is large enough, the first step in the mean-field technique is that each SBS $n$ may regard the channels' gains from all interferers (i.e. the non-zero elements of the $n^{\text {th }}$ row in $\mathbf{H i}$ ) as all possible values of a random variable $h_{-n}$ whose distribution is $F_{h_{-n}}$ with a mean value of $\bar{h}_{-n}$. The second step is to coin a distribution $F_{p_{-n m}}$ for the transmit power across interferers ${ }^{6}$ with a mean value of $\bar{p}_{-n m}$. If this distribution happens to be true, and since $p_{k m}, \forall k \neq n$, is usually independent of $\left|h_{n k m}(t)\right|$, then according to the mean-field approximation theory [15], we can say the following about the resultant interference on each radio subchannel $m$ :

$$
\text { As } N \longrightarrow \infty, \text { as } \sum_{k=1 ; k \neq n}^{N} p_{k m}\left|h_{n k m}\right| \longrightarrow N^{\prime} \bar{p}_{-n m} \bar{h}_{-n m}
$$

\footnotetext{
${ }^{6}$ The components of vector $\mathbf{p}_{-n m}$ will be regarded as if they are drawn from $F_{p_{-n m}}$.
} 
where $N^{\prime}=N-1$. Using the conjectured interference in (5.6), each player $n$ solves the problem, as described below, to make the optimal decision $p_{n m}^{*}$ with its distribution

across UEs: $\hat{F}_{p_{n m}}$. If this actual distribution matches what has been coined earlier (i.e. $F_{p_{-n m}}$ ), then $p_{n m}^{*}, \forall n$, is an oblivious equilibrium $[12,16,68]$.

If we succeed in finding such a distribution, whose mean parameter is $\bar{p}_{-n m}$, then by (5.6), the approximated interference is going to be frequency selective interference due to the fact that $\bar{h}_{-n m}$ is different across subchannels. Therefore, in addition to the frequency diversity in the desired channel, we have a new dimension of frequency diversity, which is interference frequency diversity. Both kinds of diversity are to be jointly exploited.

In brief, to correctly approximate the resultant interference as in (5.6), we need to coin a distribution for the transmit power across transmitters, which matches the distribution of the actual transmit power found by optimizing the utility of each user.

\subsubsection{Estimation of the Interference Channels' Average $\bar{h}_{-n m}$}

This subsection treats the issue of estimating $\bar{h}_{-n m}, \forall m \in \mathcal{M}^{S}$. Before the start of the game, there will be a short training period to estimate the value of $\bar{h}_{-n m}$. During the training, each interferer will send a predetermined message with a predetermined common transmit power $p_{t}$, so that the received power at base station $n$ will be:

$$
p_{n r}=p_{t} h_{n m}+I_{n m}+\sigma^{2},
$$

where

$$
I_{n m}=\sum_{k} p_{t} h_{n k m}=p_{t} \sum_{k} h_{n k m} \approx N^{\prime} p_{t} \bar{h}_{-n m}
$$


The last approximated equality is due to mean-field approximation, as described in the previous subsection. Combining (5.7) and (5.8) yields

$$
\bar{h}_{-n m} \approx \frac{p_{n r}-p_{t} h_{n m}-\sigma^{2}}{N^{\prime} p_{t}}
$$

where $p_{n r}$ is measured, $h_{n m}$ is previously known, and $p_{t}$ is a predetermined value. Owing to the law of large numbers and our i.i.d. assumptions on the channel gains, $\bar{h}_{-n m}$ can be considered the long-run average of the interference channels. This is similar to the long-run average method explained in [11]. This property of $\bar{h}_{-n m}$ is considered another merit of this approach, since it can help stabilize the system against environmental changes. Moreover, the value of $\bar{h}_{-n m}$, along with the value of $h_{n m}$, can be used jointly to design the scheduling protocol of each SBS in case there are multiple users in each small cell.

\subsubsection{Conjecture of the Interferers' Transmit Power Average $\bar{p}_{-n m}$}

Each user $n$ is going to conjecture an $N^{\prime} X M$ matrix $P$, which represents the interferes' transmit powers. Since the number of interferers $N^{\prime}$ is very large, we can regard the elements of every column $m$ of $P$ as all possible values of a dummy random variable, whose distribution is $F_{p_{-n m}}$. Therefore, the elements of each column will be drawn from the coined distribution $F_{p_{-n m}}$, with the condition that the sum of each row of $P$ is the total allowable power for each $\operatorname{SCU} p_{\text {total }}^{(F)}$. Then, the mean value of the interferers' powers on subchannel $m$ (i.e. $\bar{p}_{-n m}$ ) is found by taking the average of the $m^{t h}$ column in $P$. In other words, $\overline{\mathbf{p}}_{-n}=\left[\bar{p}_{-n 1}, \ldots, \bar{p}_{-n M}\right]$ is found by taking the average of $P$ across its rows. A suggestion of the coined distribution is discussed in Section 5.4. 


\subsubsection{Utility Functions}

We use a logarithmic utility function for all players. It is as follows:

$$
\begin{gathered}
u_{n}\left(p_{n}\right)=\sum_{m \in \mathcal{M}^{S}}\left[\ln \left(1+\frac{p_{n m}\left|h_{n m}\right|}{\beta_{n}\left(N^{\prime} h_{-n m} \bar{p}_{-n m}+\sigma_{n m}^{2}\right)}\right)\right], \\
\forall n \in \mathcal{N} \backslash\{0\} .
\end{gathered}
$$

Now each player $n$ seeks to maximize its utility function, which is not directly affected by any individual competitor ${ }^{7}$. Instead, the utility is only affected by the collection of all other players through the mean-field value $\bar{h}_{-n m}$. This problem can be solved in a decentralized manner.

\subsubsection{Decentralized Solution}

Using the statistical data of the interferers' channel and applying (5.6) helps us extend the optimization technique, which was used for power allocation in the noise limited system, to include the interference limited system. Therefore, if each player appropriately coins a distribution for the interferers' powers, each SBS $n$ can find its optimal transmitted power vector $\mathbf{p}_{n}^{*}$ in a distributed fashion by solving the following concave optimization problem.

$$
\begin{array}{ll}
\underset{p_{n m}, \forall m \in \mathcal{M}^{S}}{\operatorname{maximize}} & \sum_{m \in \mathcal{M}^{S}}\left[\ln \left(1+\frac{p_{n m}^{*}\left|h_{n m}\right|}{\beta_{n}\left(N^{\prime} \cdot \bar{h}_{-n m} \cdot \bar{p}_{-n m}+\sigma_{n m}^{2}\right)}\right)\right] \\
\text { subject to } & \sum_{m \in \mathcal{M}^{S}} p_{n m}^{*}=p_{\text {total }}^{(F)},(\text { Total Power Const) } \\
& p_{n m}^{*} \geq 0, \forall m \in \mathcal{M}^{S} . \quad \text { (Nonnegativity) }
\end{array}
$$

Forming the Lagrangian of the above optimization problem, and solving for $p_{n m}^{*}$

\footnotetext{
${ }^{7}$ The idea that the action of an individual has almost no impact on the game outcome lies in the core of the mean-field game theory.
} 
yields

$$
p_{n m}^{*}= \begin{cases}\frac{1}{\lambda_{n}}-\frac{\beta_{n}\left(N^{\prime} \cdot \bar{h}_{-n m} \cdot \bar{p}_{-n m}+\sigma_{m}^{2}\right)}{\left|h_{n m}\right|} & \text { if }\left|h_{n m}\right|>h_{n m}^{(l)} \\ 0 & \text { if }\left|h_{n m}\right| \leq h_{n m}^{(l)}\end{cases}
$$

$\forall m \in \mathcal{M}^{S}$

where $h_{n m}^{(l)}=\lambda_{n} \beta\left(N^{\prime} \cdot \bar{h}_{-n m} \cdot \bar{p}_{-n m}+\sigma_{m}^{2}\right)$ is the minimum acceptable desired channel level, and $\lambda_{n}$ is the Lagrangian multiplier. $p_{n m}^{*}$ can be written simply as

$$
p_{n m}^{*}=\frac{1}{2}\left[\frac{1}{\lambda_{n}}-\phi_{n m}+\left|\frac{1}{\lambda_{n}}-\phi_{n m}\right|\right]
$$

where

$$
\phi_{n m}=\frac{\beta_{n}\left(N^{\prime} \cdot \bar{h}_{-n m} \cdot \bar{p}_{-n m}+\sigma_{m}^{2}\right)}{\left|h_{n m}\right|} .
$$

$\lambda_{n}$ can be found by numerically solving the following equation (5.14) resulted from substituting equation (5.13) into the total power constraint.

$$
2 p_{\text {total }}^{(F)}=\sum_{m \in \mathcal{M}^{S}}\left[\frac{1}{\lambda_{n}}-\phi_{n m}+\left|\frac{1}{\lambda_{n}}-\phi_{n m}\right|\right]
$$

Note that according to $(5.12), p_{n m}^{*}$ adapts to $\left|h_{n m}\right|, \bar{h}_{-n m}$ as well as to $N^{\prime}$. If, for example, the number of interferers increases, $p_{n m}^{*}$ will decrease. This adaptation will maintain interference at minimum levels throughout the network.

Once all the players had found their optimal power vector $\mathbf{p}_{n}^{*}$, we have a distributed $N X M$ matrix $\hat{P}$, whose row vector $n$ is the optimal power vector $\mathbf{p}_{n}^{*}$ selected by SBS $n$. If the empirical distribution of the column $m$ elements matches the assumed distribution coined earlier, then $\left(\mathbf{p}_{1}^{*}, \mathbf{p}^{*} \mathbf{p}_{2}^{*}, \ldots, \mathbf{p}_{N}^{*}\right)$ is an equilibrium point. The proposed scheme using MFG is summarized in Algorithm 3. 


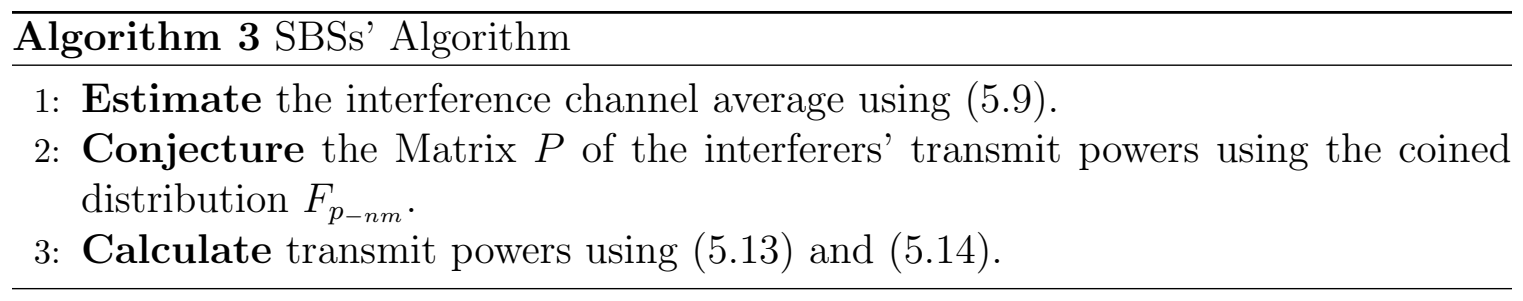

\subsection{Simulation Results and Discussions}

The goal of this section is to evaluate the performance of the proposed algorithms. We simulated an area $A$ consisting of an MBS in its center and several small cells. For small cells, we examined two topologies. The first topology is random and models the actual case of small cells, where SBSs are installed by the end users. The second topology models the case of organized small cells (e.g. pico cells), where operators carefully choose the locations of BSs.

Based on the simulation parameters shown in Table 5.1, we found that when MBS applies Algorithm 2 to meet the required QoS, it occupies on average 10.0860 subchannels. More precisely, the $95 \%$ confidence interval of the number of subchannels selected by the macrocell (i.e. $\left|\mathcal{M}^{C}\right|$ ) is $[9.2956,10.8764]$. Hence, $96 \%$ of the system resources are left for small cells. Moreover, since Algorithm 2 supports green policy at the macrocell level, we found that the $95 \%$ confidence interval of the macrocell energy efficiency is $[120.72,142.56] \mathrm{kb} / \mathrm{J}$.

In the subsections below, we show the performance of the mean-field game approach in combating co-layer interference. Moreover, all small BSs have full access to all subchannels in $\mathcal{M}^{S}$.

\subsubsection{Random Network Topology}

In this scenario, all SBSs are deployed randomly within the total area. In addition, we equipped each SBS with our resource allocation scheme and repeated the simulation for different cases. 
As indicated in Section 5.3, we need to coin a distribution for the transmit power of the interferers so that each transmitter can estimate the expected interference power. With that estimation, each transmitter will find its optimal transmit power using (5.13) and (5.14). As a consistency condition, we require that the distribution of the actual power, which has been found optimally be each transmitter, to match the coined distribution that helped to estimate the interference.

To coin the distribution of power across the transmitters, we tried different distributions. The distribution that most closely matches that of the optimal selected power is the uniform distribution over a set of vectors whose components' sum is $p_{\text {total }}^{(F)}$. In other words, all possible transmit power vectors whose components' sum is $p_{\text {total }}^{(F)}$ have the same chance of being selected. Note that the condition for fixing the sum of the components' makes the distribution of each component different from the uniform distribution. In fact, each component's distribution appears to be a truncated exponential distribution (i.e. $\left.\operatorname{TEXP}\left(\mu, p_{\text {total }}^{(F)}\right)\right)$. From Fig. 5.1, we can observe that when we apply this distribution, the users select power levels whose distribution is closely related to the applied (coined) one. To validate our scheme, we fitted these distributions to a truncated exponential distribution. The figure shows how the fitted distributions of the coined and actual powers are very close to each other.

Furthermore, our target is to examine the average spectral efficiency per cell, as a performance measure. The numerical results for different scenarios are shown in Fig. 5.2. We can see that, in all cases, the spectral efficiency per cell decreases as the number of small cells increases in the total area. This is simply because the interference power at any BS increases proportionally to the number of interferers. Moreover, an increase in the area $A$ will result in an increase in the spectral efficiency. Such an increase in the area will potentially make the small cells separate from each other and hence, less interference will be experienced by each BS. Finally, when the number of radio resources increases, there is an increase in the spectral efficiency, 
Table 5.1: Simulation parameters.

\begin{tabular}{|c|c|}
\hline Parameters & Value \\
\hline \hline System frequency & $2 \mathrm{GHz}$ \\
\hline System bandwidth & $5 \mathrm{MHz}$ \\
\hline No. of data subchannels & 300 \\
\hline Subchannel BW & $15 \mathrm{KHz}$ \\
\hline$p_{\text {total }}^{(C)} p_{\text {total }}^{(F)}$ & $45 \mathrm{dBm}, 30 \mathrm{dBm}($ respectively) \\
\hline Small cell radius $r_{F}$ & $30 \mathrm{~m}$ \\
\hline MUE required rate: $R_{0}$ & $3 \mathrm{Mbps}$ \\
\hline Reqired BER & $10^{-6}$ \\
\hline Noise power spectral density & $-174 \mathrm{dBm} / \mathrm{Hz}$ \\
\hline Shadow fading deviation & $8 \mathrm{~dB}$ \\
\hline Small scale fading & Rayleigh fading \\
\hline
\end{tabular}

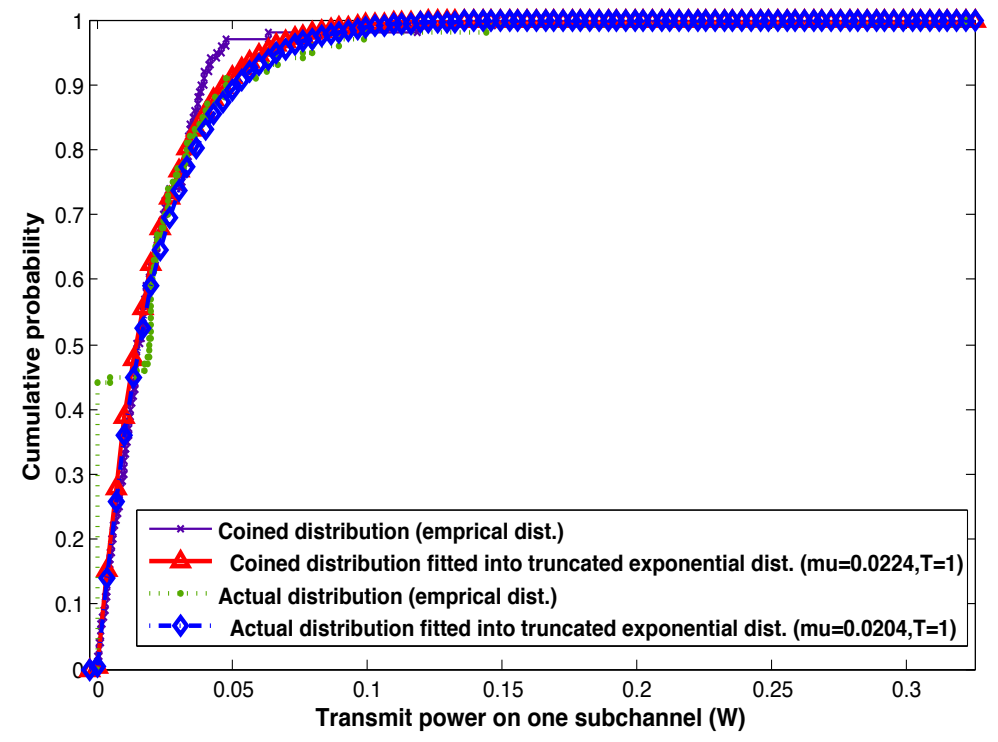

Figure 5.1: The coined distribution of the transmit power versus the distribution of the actual transmit power obtained by (5.13). 


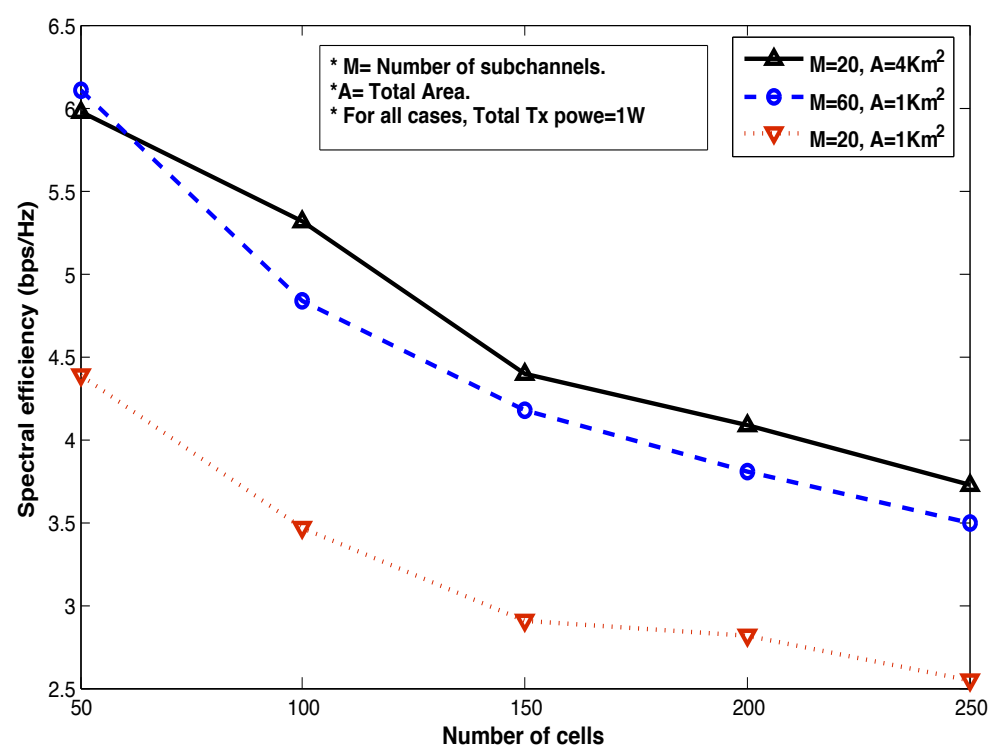

Figure 5.2: Spectral efficiency in different scenarios.

owing to frequency diversity. However, as the number of interferers increases, the frequency diversity gain diminishes.

\subsubsection{Organized Network Topology}

In the hexagonal network topology, we compared the performance of a system equipped with our proposed scheme against a system using the frequency reuse technique of factor 3 (Reuse-3). The organized topology is realistic for cases where operators plan the network deployment. In Reuse-3, each small BS has access to a third of the total bandwidth that is different than that of its neighboring BSs. For fair comparison, each SBS in the Reuse-3 system is optimally adaptive with the desired channels. In the system that employs our scheme, each SBS has access to the whole spectrum.

The results are shown in Fig. 5.3. In all cases, we can see that the spectral efficiency of the system employed with our scheme is almost double that of a system using the Reuse-3 scheme. The reason is that our scheme cleverly uses the whole 


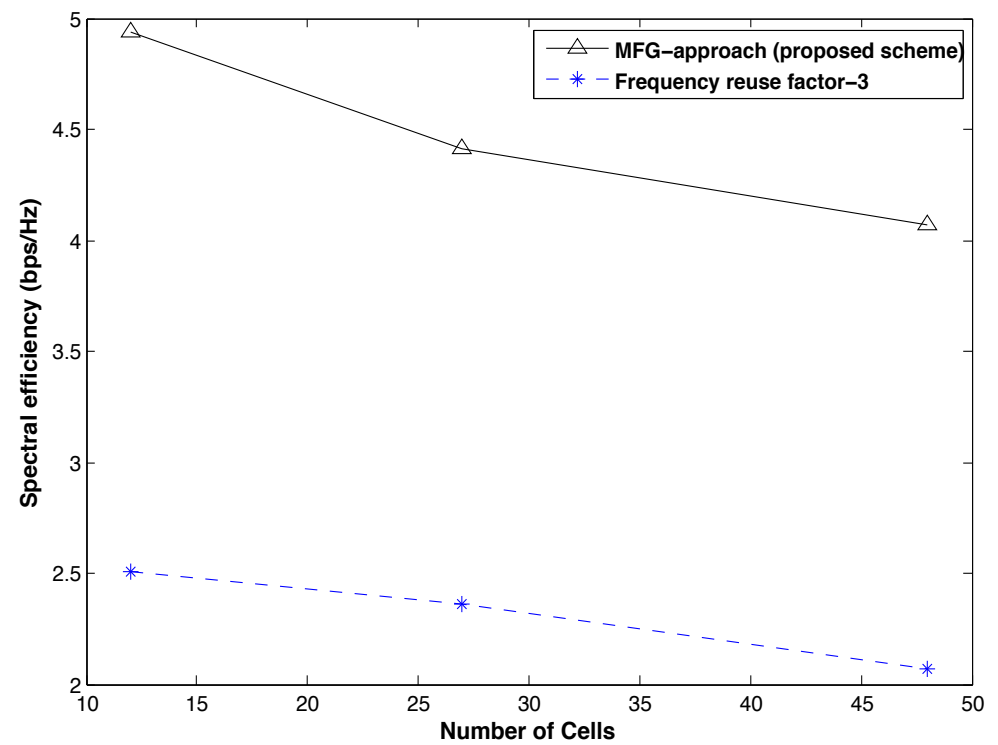

Figure 5.3: Spectral efficiency comparison between the MFG-based approach and the Reuse-3 technique. TotalArea $\approx N \pi r_{F}^{2}$.

spectrum by exploiting the interference diversity over the radio resources. It statistically approximates the interference in the next slot for every subchannel, and then optimally distributes the limited power over all subchannels. Again, the key step is to ensure that the statistics of the approximations match those of the optimally selected ones.

\subsection{Conclusions}

To meet the increasing demand for high-speed wireless services, spectrum reuse through dense small cells deployment is becoming increasingly inevitable due to its promising payoff, especially when compared to other solutions. However, one of the most tremendous challenges that accompany the anticipated payoff is co-channel interference. Optimal interference control in such a dense network, using centralized and global approaches, is enormously prohibitive due to scalability issues as well as overwhelming overhead costs. 
Since MBS is usually less efficient (spectrally and energetically) than SBS, we proposed a scheme by which the MBS is assigned a minimum number of radio resources, which are just enough to satisfy the required QoS. Then, the remaining subchannels are assigned to all SBSs. To combat the co-layer interference, we proposed a mean-field game-based scheme, in which the total power of each player is optimally distributed over the subchannels. By exploiting the statistics of the interfering channels, along with applying mean-field approximation, a new dimension of frequency diversity is created in the interference part. Higher spectral efficiency is attained by jointly taking advantage of both frequency diversities in the desired channel as well as in the interference channel. 


\section{Chapter 6}

\section{Resource Allocation and Interference Management Based on Full Spectrum Sharing for Hyper-Dense HetNets}

This chapter examines full spectrum sharing in a dense heterogeneous network of one MBS and a large number of SBSs, which are all located within the macrocell area. In particular, we aim to find a resource allocation and interference management method in which the spectrum resources are fully shared between all BSs, including the MBS.

As shown in Chapter 3, the interference problem in HetNets has drawn a lot of research attention in recent years. Despite the considerable amount of research conducted in this field, the majority of the proposed schemes are based on deterministic approaches, which assume that the perfect information of all involved channels is available. This assumption may not be realistic for the following reasons. In practical heterogeneous networks, the SBSs owned by individual subscribers are connected to the MBSs owned by operators using backhaul communication links that usually have limited-capacity due to their deployment costs [32,51]. Moreover, these BSs and their users may not have protocols to share channel information between each other. Even if this kind of protocol exists, appropriate incentive mechanisms are still 
needed in order to ensure that the network devices truthfully exchange information, since their objectives may not be aligned [52]. Furthermore, even if they are willing to share information, the shared information is very likely to be outdated due to the limited capacity of the backhaul links, which can result in significant performance degradation $[53,54]$. As a result, complete channel information may be unavailable to the others.

Imperfect channel information has a significant impact on the performance of, not only heterogeneous networks, but also wireless networks in general. Indeed, the capacity of channels with imperfect channel information is largely unknown in wireless networks. Game theory has well-developed mechanisms to address the impacts of imperfect channel information, which will give insights into the problems related to imperfect channel information from a new perspective [55].

In this chapter, using recent advances in mean-field game theory [56], a novel game theoretic approach for interference control in HetNets is introduced. The mean-field game theory provides a powerful mathematical tool for problems with a large number of players. It has been successfully used by economists, socialists, and engineers in different areas, among others [57]. In communication networks, several researchers have tried to use mean-field game theory to solve the energy efficiency [58] and medium access control [59] problems. To the best of our knowledge, using the mean-field game theoretic approach for interference control in HetNets was not considered in any existing work.

The rest of this chapter is organized as follows. The system model and problem formulations are described in Section 6.1. In Section 6.2, the underlay game problem is addressed and analyzed. The overlay problem is then treated and analyzed in Section 6.3. Subsequently, simulation results are discussed in Section 6.4. Finally, we conclude this work in Section 6.5. 


\subsection{System Description and Problem Formulation}

We consider a discretized time HetNet system with orthogonal frequency division multiplexing (OFDM). In this system, as shown in Fig. 6.1, a macrocell area consists of one MBS and its $J$ associated MUs, as well as $N$ SBSs and their associated small cell users (SCUs). The set of available radio subchannels is $\mathcal{M}=\{1, \ldots, m, \ldots, M\}$, where each subchannel is accessible by each BS.

In this research, the medium access control (MAC) protocol in SBSs can be chosen arbitrarily. If any MAC scheme (e.g., proportional fair scheduler) is adopted, each SBS schedules its users first, and then each SCU in that cell would play the game on each subchannel assigned to him. For the sake of simple description, we consider TDMA as the MAC protocol in SBSs, hence, one receiving user per small cell per time slot. Accordingly, for a given time slot $t, \mathcal{N}=\left\{S B S_{1}, S B S_{2}, \ldots, S B S_{N}\right\}$ and $\mathcal{N}_{S C U}=$ $\left\{S C U_{1}, S C U_{2}, \ldots, S C U_{N}\right\}$ are the sets of SBSs and SCUs, respectively. On the other hand, $\mathcal{J}=\left\{M U_{1}, M U_{2}, \ldots, M U_{J}\right\}$ is the set of macro users that can orthogonally associate with their MBS whose index is 0. Considering the downlink, the signal to interference plus noise ratio (SINR) at $M U_{j}$ over the $m^{\text {th }}$ subchannel is:

$$
\gamma_{j m}(t)=\frac{p_{0 m}(t) h_{j 0 m}(t)}{\sum_{k=1}^{N} p_{k m}(t) h_{j k m}(t)+B N_{o}}=\frac{p_{0 m}(t) h_{j 0 m}(t)}{I_{j m}(t)+B N_{o}},
$$

where $\gamma$ is the SINR. $p_{0 m}$ and $p_{k m}$ are the transmit power over $m^{\text {th }}$ subchannel from the MBS and the interfering $S B S_{k}$, respectively. In general, $h_{j k m}$ is the $m^{\text {th }}$ subchannel gain from BS in cell $k$ to the $j^{\text {th }}$ MU. Hence, $h_{j 0 m}$ and $h_{j k m}$ are the power gains of the desired channel from MBS and the interfering channel from $S B S_{k}$, respectively. $B$ and $N_{o}$ are the subchannel bandwidth and the noise power spectral density, respectively . $I_{j m}$ is the total interference received by $M U_{j}$ on subchannel $m$. It is assumed that the MBS knows the desired channel gain to each MU.

On the other hand, the SINR at $S C U_{n}$ over the same subchannel $m$ and at the 


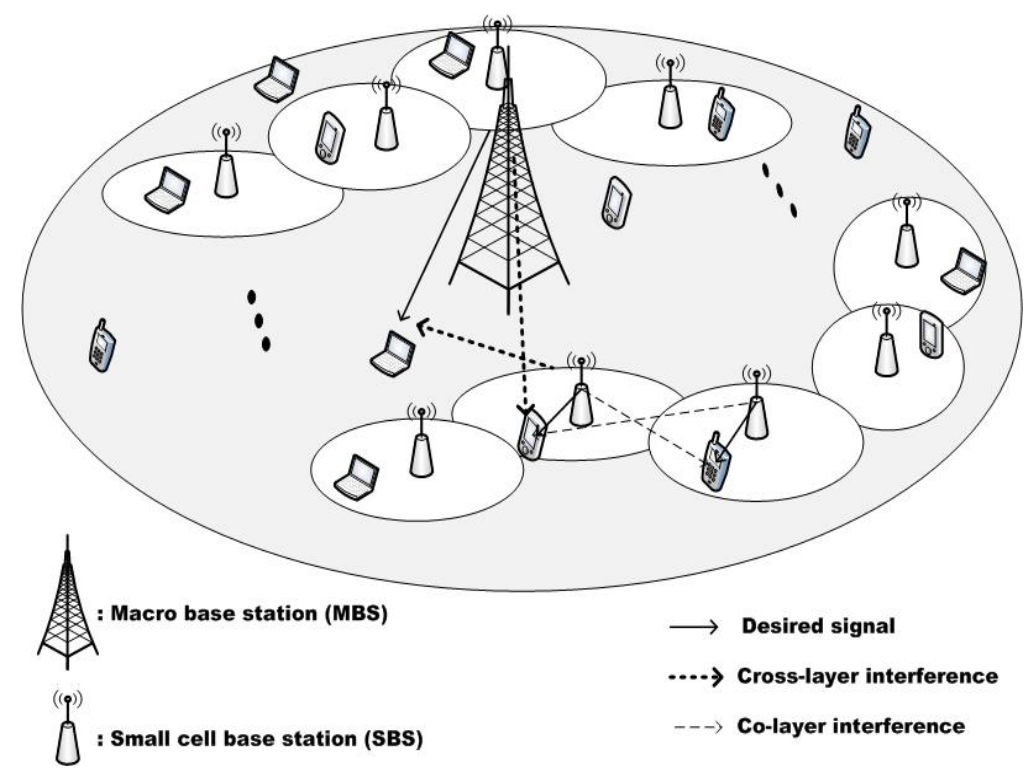

Figure 6.1: A heterogeneous wireless network.

same time $\operatorname{slot}^{1}$ is

$$
\begin{aligned}
\gamma_{n m}(t) & =\frac{p_{n m}(t) h_{n n m}(t)}{\sum_{\substack{k=1 \\
k \neq n}}^{N} p_{k m}(t) h_{n k m}(t)+p_{0 m}(t) h_{n 0 m}(t)+B N_{o}} \\
& =\frac{p_{n m}(t) h_{n n m}(t)}{I_{n m}(t)+B N_{o}},
\end{aligned}
$$

where $p_{n m}, p_{k m}, p_{0 m}$ are the transmit powers over $m^{\text {th }}$ subchannel from the serving $S B S_{n}$, interfering $S B S_{k}$ and MBS, respectively. $h_{n n m}$ is the desired channel gain of $S C U_{n}$ from its serving base station $S B S_{n} . h_{n k m}$ and $h_{n 0 m}$ are the $S C U_{n}$ interfering channels from $S B S_{k}$ and MBS. In an abuse of notation, we will use the shorthand $h_{n m}(t)=h_{n n m}(t)$ which refers to the $m^{t h}$ subchannel gain between $S C U_{n}$ and its serving BS. We assume the desired channel gains $h_{n m} \forall n \in \mathcal{N}$ are independent and identically distributed (i.i.d). The cross-channel gains are also assumed i.i.d. Moreover, It is assumed that each SBS knows the desired channel gain of its associated user. $I_{n m}$ is the sum of cross-layer interference and the co-layer interference received

\footnotetext{
${ }^{1}$ For the sake of simplicity, the argument $[t]$ will be omitted in the subsequents.
} 
by $S C U_{n}$ on subchannle $m$.

Assuming the channel fading is characterized by Rayleigh distribution, we consider $h_{n m}(t)$ as an exponentially distributed random process whose parameter is $\mu_{n}$, which is equal to the reciprocal of the path gain.

In the following two subsections, we formulate the interference management issue in this HetNet system as two nested problems: an overlay problem at the MBS level and underlay problem at the SBS level.

\subsubsection{Overlay Problem Formulation}

Since mobile operators are committed to providing anywhere, anytime service, the quality of service (QoS) of MUs has to be guaranteed. Therefore, we consider the scenario that satisfying the MUs has a higher priority over maximizing the network sum rate by relying heavily on the small cells. Thus, the MBS would first decide its transmit power level on each subchannel such that MUs are just satisfied without any more provision. This is for minimizing cross-layer interference to SBSs. Then, given the interference coming from MBS, each SBS would react by choosing a transmit power that maximizes its own utility. We will later demonstrate that the MBS's decisions take into account the reaction of the SBSs.

Assuming for now that the MBS can anticipate the SBSs reaction, the MBS problem can be formulated as follows: 


$$
\begin{aligned}
& \underset{X_{j m}, p_{0 m}}{\operatorname{minimize}} \sum_{m \in \mathcal{M}} p_{0 m} \\
& \text { subject to } \\
& \sum_{m \in \mathcal{M}} B \log _{2}\left(1+\frac{p_{0 m} h_{j 0 m}}{\beta_{j}\left(\sum_{k=1}^{N} p_{k m} h_{j k m}+B N_{o}\right)}\right) X_{j m} \geq R_{j}, \forall j, \\
& \sum_{m \in \mathcal{M}} p_{0 m} \leq p_{\text {total }}^{(C)}, \\
& \sum_{j \in \mathcal{J}} X_{j m} \leq 1, \forall m, \\
& X_{j m} \in\{0,1\}, \forall m \& j, \\
& p_{0 m} \geq 0, \forall m,
\end{aligned}
$$

where $X_{j m}$ is a binary variable whose value is 1 when the $m^{\text {th }}$ subchannel is assigned to the $M U_{j}$ and 0 otherwise. $B$ is the subchannel bandwidth, $R_{j}$ is the $M U_{j}$ minimum required data rate, and $p_{\text {total }}^{(C)}$ is the MBS total transmit power. Moreover, $\beta_{j}=$ $-\ln \left(5 B E R_{j}\right) / 1.5$ is an application-dependent positive factor that takes into account the required bit error rate (BER) $[8,9]$. The objective function is meant to minimize the cross-layer interference caused by the MBS while the first constraint in (6.3.a) is meant to guarantee MUs' satisfaction. The left hand side of the first constraint (6.3.a) analytically captures the effect of the adaptive modulation and coding scheme [8] [9]. The second constraint (6.3.b) takes into account the limited power budget of the MBS whereas the third constraint (6.3.c) is to ensure that MUs are orthogonally scheduled, and hence free intracell interference. (6.3.d) and (6.3.e) are the space of the variables $X_{j m}$ and $p_{0 m}$, respectively. 


\subsubsection{Underlay Problem Formulation}

Small cell base stations, which transmit over the same subchannel $m \in \mathcal{M}$, mutually affect each other via inter-cell interference. Therefore, we consider an underlay game in which each SBS $n \in \mathcal{N}$ is a rational player competing with the other SBSs over the system resources, given the interference coming from the MBS. Before SBSs plays the game, each SBS measures the amount of the cross-layer interference caused by the MBS. Therefore, from now on we will combine the interference caused by the MBS and the noise power as: $p_{0 m} h_{n 0 m}+B N_{o}=\sigma_{n m}^{2}$.

In this work, a non-cooperative strategic game $\mathcal{G}=\left(\mathcal{N},\left\{\mathcal{P}_{n m}\right\}_{n=1}^{N},\left\{u_{n m}\right\}_{n=1}^{N}\right)$ is considered to model the interaction among SBSs, where:

- $\mathcal{N}$ is the set of players that are considered here to be the SBSs.

- $\mathcal{P}_{n m}=\left[0, p_{m}^{\max }\right]$ is a continuous set of transmit power which represents the action space of the $n^{\text {th }}$ player on the $m^{\text {th }}$ subchannel. We consider here a symmetric game in which the available action sets for all players are identical. $p_{m}^{\max }$ is the maximum transmit power over subchannel $m$.

- $u_{n m}$ is the $n^{\text {th }}$ player's utility, which is a mathematical expression that quantifies the level of satisfaction a user gets by using the $m^{\text {th }}$ subchannel.

It is reasonable to suppose that the level of satisfaction of every user is closely related to the achievable rate, which is directly proportional to the transmit power. However, such a relation would encourage each player to transmit with full power leading to an uncontrolled interference. Therefore, in addition to the achievable rate as a positive part of the utility function representing the level of reward, we have to add a negative part that corresponds to a cost of using the system resources (or one can look at it 
as a penalty for interfering other users). Thus, the utility function of every player is:

$$
u_{n m}=\alpha_{n} \ln \left(1+\frac{\gamma_{n m}}{\beta_{n}}\right)-\lambda_{n m} p_{n m}, \quad p_{n m}, \gamma_{n m} \geq 0
$$

where $\alpha_{n}$ is a fairness weighting factor that ensures that end users relatively have fair share of the system resources. It is the reciprocal of the exponential moving average $(E M A[t-1])$ of the achieved rates till the previous time slot, averaged over the last window of $\rho$ time slots, that is;

$$
\frac{1}{\alpha_{n}[t]}=E M A_{n}[t-1]=\frac{1}{\rho} \cdot r_{n}[t-1]+\left(1-\frac{1}{\rho}\right) \cdot E M A_{n}[t-2],
$$

where $r_{n}[t]$ is the achieved rate at time slot $t$. Furthermore, $\lambda_{n m}$ is a pricing factor in bps $/(\mathrm{Hz} \cdot \mathrm{watt})$, and $\beta_{n}$ is an application-dependent positive factor that takes into account the required $\mathrm{BER}, \beta_{n}=-\ln \left(5 B E R_{n}\right) / 1.5[8,9]$. To facilitate easy presentation without loss of generality, we can $\operatorname{drop} \beta$ by simply assuming that all users require the same BER, and all are equipped with a powerful coding that achieves Shannon capacity (e.g., Polar or Turbo coding). Thus,

$$
u_{n m}=\alpha_{n} \ln \left(1+\frac{p_{n m} h_{n m}}{\sum_{\substack{k=1 \\ k \neq n}} p_{k m} h_{n k m}+\sigma_{n m}^{2}}\right)-\lambda_{n m} p_{n m}
$$

\subsection{Underlay Game Treatment}

In this section, the underlay problem is analyzed and solved. In addition, an algorithm that attains the solution in a distributed manner is proposed.

When the number of small cells is large, the utility function in (6.5) would cause an analytical problem as the curse of dimensionality kicks in. Indeed, the game would be beyond traditional mathematical treatment for both tracking the required information and computing the best decisions. In fact, a distributed algorithm would 
take a very long time to converge if the number of players is very large. Therefore, we propose the following solution.

\subsubsection{System Decoupling Using Mean-Field Theory}

Considering a representative SBS $n$ and its associated SCU, let $\bar{h}_{-n m}$ be the average of $h_{n 1 m}, \ldots, h_{n(n-1) m}, h_{n(n+1) m}, \ldots, h_{n N m}$. In a system with a large number of interferers, we might expect that the time domain fluctuations of interference channels (i.e., $\left.h_{n k m} \forall k\right)$ average out. Therefore their average $\bar{h}_{-n m}$ would remain roughly constant over all outcomes in the sample space, and hence $\bar{h}_{-n m}$ is in fact, a long-run average [11].

As mentioned above, all players are coupled with each other via the interference term. Since the power $p_{k m}$ of each interferer $k \neq n$ is independent of $h_{n k m}$, then we can safely use the MFA method to decouple the system by having the following:

$$
\begin{gathered}
I_{n m}=\sum_{k=1 ; k \neq n}^{N} p_{k m} h_{n k m} \approx N^{\prime} \bar{h}_{-n m} \bar{p}_{m} \\
\text { as } N^{\prime} \longrightarrow \infty,
\end{gathered}
$$

where $N^{\prime}=N-1$, and $\bar{p}_{m}=E\left[p_{k m}\right]$ is the average transmit power across all interferers $^{2}$. The validity of the above approximation can be shown as follows.

$$
I_{n m}=\frac{N^{\prime}}{N^{\prime}} \sum_{k=1 ; k \neq n}^{N} p_{k m} h_{n k m} .
$$

From the probability theory, if $N^{\prime} \rightarrow \infty$, the sample (arithmetic) average approaches the statistical mean. Therefore, $I_{n m} \approx N^{\prime} E\left[p_{k m} h_{n k m}\right]=N^{\prime} \bar{h}_{-n m} \bar{p}_{m}$. The last equality is due to the independence between $p_{k m}$ and $h_{n k m}$.

Owing to MFA, the impact of the other $S B S$ on cell $S B S_{n}$ is replaced by a virtual

\footnotetext{
${ }^{2}$ This amount could be an operation parameter dictated by the system, as we will see below.
} 
field, whose value is $N^{\prime} \bar{h}_{-n m} \bar{p}_{m}$ that represents the effect of the interfering population. Thus, the utility function in (6.5) can be approximated by:

$$
u_{n m}=\alpha_{n} \ln \left(1+\frac{p_{n m} h_{n m}}{N^{\prime} \bar{h}_{-n m} \bar{p}_{m}+\sigma_{n m}^{2}}\right)-\lambda_{n m} p_{n m}
$$

Because (6.8) is applicable to every player $n$, then $\bar{p}_{m}$ should be a common value that maintains players artificially coupled.

The issue of estimating $\bar{h}_{-n m}, \forall m \in \mathcal{M}^{S}$ is treated the same way as in Subsection 5.3 .1 .

\subsubsection{Game Outcome and Condition}

While all players are kept technically correlated through $\bar{p}_{m}$, they have been mathematically decoupled using MFA. Therefore, game analysis and outcome can be worked out considering only one generic representative player $S B S_{n}$. Thus, for maximizing the utility function in (6.8), $S B S_{n}$ would select its transmit power to be:

$$
p_{n m}^{*}=\left\{\begin{array}{ll}
\frac{\alpha_{n}}{\lambda_{n m}}-\frac{N^{\prime} \bar{h}_{-n m} \bar{p}_{m}+\sigma_{n m}^{2}}{h_{n m}}, & h_{n m} \geq h_{n m}^{(l)} \\
& \\
0 & \text { Otherwise }
\end{array} \quad \forall n,\right.
$$

where $h_{n m}^{(l)}=\frac{\lambda_{n m}}{\alpha_{n}}\left[N^{\prime} \bar{h}_{-n m} \cdot \bar{p}_{m}+\sigma_{n m}^{2}\right]$ is a cut-off threshold of the desired channel gain, below which the transmitter of $S B S_{n}$ will not transmit. Accordingly, (6.9) can 
be written as:

$$
p_{n m}^{*}= \begin{cases}\frac{\alpha_{n}}{\lambda_{n m}}\left(1-\frac{h_{n m}^{(l)}}{h_{n m}}\right), & h_{n m} \geq h_{n m}^{(l)} \\ & \text { Otherwise. }\end{cases}
$$

Note that (6.10) represents a homogeneous strategy for all SBSs, and hence maintaining the symmetry property among all players. Eq. (6.10) is the mean-field game outcome that would approach Nash equilibrium as $N \longrightarrow \infty$. However, in order to ensure that (6.6) works properly and hence the whole game, the statistical average of the transmit power of $S B S_{n}$ (i.e., $E\left[p_{n m}^{*}\right]$ ) should equal to the average power across all its interferers (i.e., $\bar{p}_{m}$ ). This is called the consistent condition of the mean-field approximation. Thus,

$$
E\left[p_{n m}^{*}\right]=\bar{p}_{m} \quad \forall n
$$

where $E[\cdot]$ denotes a statistical mean. $(C 1)$ is a consistency condition for the meanfield approximation in (6.6) to hold, and hence for the whole game to be consistent. $E\left[p_{n m}^{*}\right]$ can be found as:

$$
E\left[p_{n m}^{*}\right]=\int_{h_{n m}^{(l)}}^{\infty} \frac{\alpha_{n}}{\lambda_{n m}}\left(1-\frac{h_{n m}^{(l)}}{h_{n m}}\right) f_{h_{n m}} d h .
$$

$f_{h_{n m}}$ is the probability density function (PDF) of the power gain of the desired channel,

$$
f_{h_{n m}}=\mu_{n} e^{-\mu_{n} h_{n m}}
$$

where $1 / \mu_{n}=E\left[h_{n m}\right]$ is the mean of the desired channel gain. Thus, condition $(C 1)$ can be rewritten as the following: 


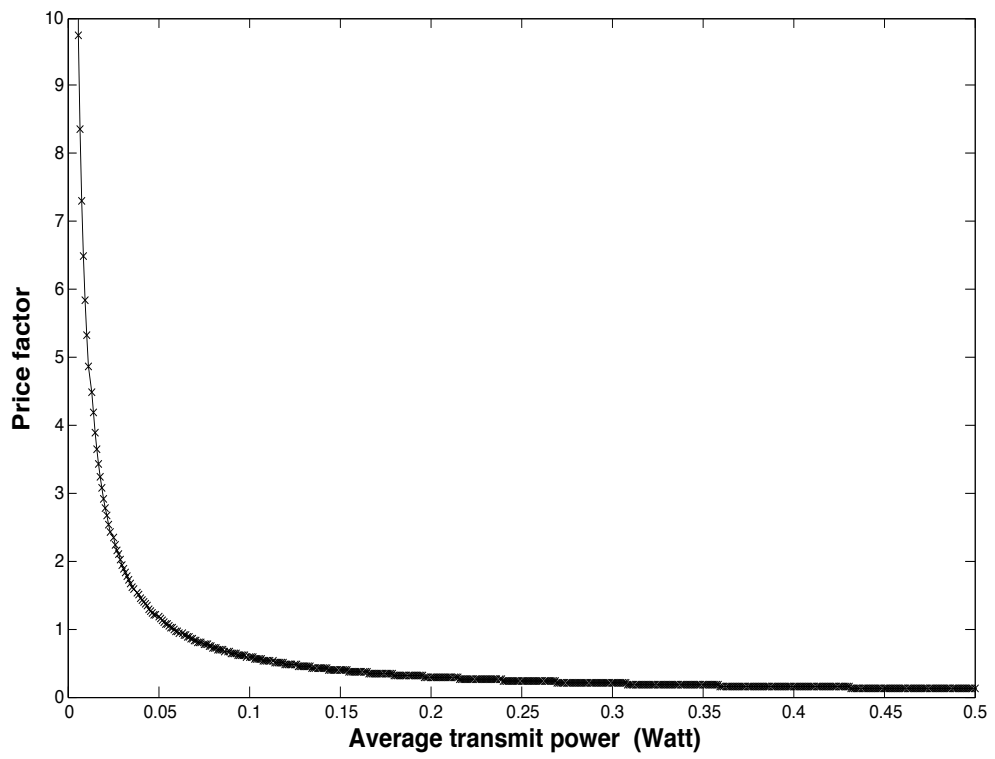

Figure 6.2: The relationship between price factor $\lambda_{n m}$ and $\bar{p}$ as described by the consistent condition $(C 1)$.

$$
\frac{\alpha_{n}}{\lambda_{n m}}\left(e^{-\mu_{n} h_{n m}^{(l)}}-\mu_{n} h_{n m}^{(l)} \cdot \mathrm{E}_{1}\left(\mu_{n} h_{n m}^{(l)}\right)\right)=\bar{p}_{m} \quad \forall n \& m
$$

where $\mathrm{E}_{1}(z)=\int_{1}^{\infty} \frac{e^{-t z} d t}{t}$ is a special function called exponential integral. It is noteworthy that the left hand side (LHS) of $(C 1)$ is a function of $\bar{p}_{m}$ via $h_{n m}^{(l)}$ (i.e., $\left.L H S=F\left(\bar{p}_{m}\right)\right)$. Therefore, $(C 1)$ can be written as $F\left(\bar{p}_{m}\right)=\bar{p}_{m}$ which suggests that $(C 1)$ has a fixed point structure.

Although (6.10) provides the optimum policy for each $S B S_{n}$, there are two variables unknown, namely, $\lambda_{n m}$ and $\bar{p}_{m}$. However, the relation between them is well described by $(C 1)$, as depicted in Fig. 6.2. Thus, if we carefully select one of them, the other one could directly be found.

\subsubsection{Underlay Interference Control Algorithm}

In this subsection, we summarize the underlay algorithm that runs in every SBS in order to control the co-layer interference in a distributed fashion. First, the MBS 
would select and broadcast the value of $\bar{p}_{m}$ to all SBS. This step will be described in the next section. Then, using $(C 1)$, each player will find its price factor $\lambda_{n m}$ that realizes that the fixed point of $F_{n}\left(\bar{p}_{m}\right)$ is $\bar{p}_{m}$ as shown in Fig. 6.3. Blue curves in Fig. 6.3 correspond to different players whose desired channels and interference channels are different, and hence their price factor $\lambda_{n m}$ would be different as well. After finding the price factor, the best transmit power $p_{n m}^{*}$ can be found via (6.10). Then, SINR $\left(\gamma_{n m}=\frac{p_{n m}^{*} h_{n m}}{N^{\prime} \bar{p}_{m} \bar{h}_{-n m}+\sigma_{n m}^{2}}\right)$ could be foreseen, and thus could be plugged into a mapping function ${ }^{3} \digamma\left(\gamma_{n m}\right)$ which calculates the anticipated data rate in subchannel $m$. Since each SBS has a limited power budget, each $S B S_{n}$ has to solve the following optimization problem to decide which subchannels it is going to utilize. This optimization is a binary linear programming which can easily be solved using Balas Additive Algorithm [60].

$$
\begin{array}{ll}
\underset{X_{n m}, \forall m \in \mathcal{M}}{\operatorname{maximize}} & \sum_{m \in \mathcal{M}} \digamma\left(\gamma_{n m}\right) X_{n m} \\
\text { subject to } & \sum_{m \in \mathcal{M}} p_{n m}^{*} X_{n m} \leq p_{\text {total }}^{(S)} \text { (Total Power Const.) } \\
& X_{n m} \in\{0,1\}, \forall m \in \mathcal{M} \text { (Variable space), }
\end{array}
$$

where $p_{\text {total }}^{(S)}$ is the total transmit power for each SBS. Problem (6.11) is optimized over the binary variable $X_{n, m}$. SBS $S_{n}$ will transmit over subchannel $m$ only if $X_{n, m}=1$. Algorithm 4 briefly describes the proposed interference control scheme that independently runs in every SBS.

\subsection{Overlay Problem Treatment}

As suggested above, the operation point $\bar{p}_{m}$ is a common known value throughout the network. Moreover, we suggest that each $M U_{j}$ is to estimate the average of the cross

\footnotetext{
${ }^{3}$ This function could be Shannon capacity or the practical adaptive modulation and coding (AMC) mapping function.
} 


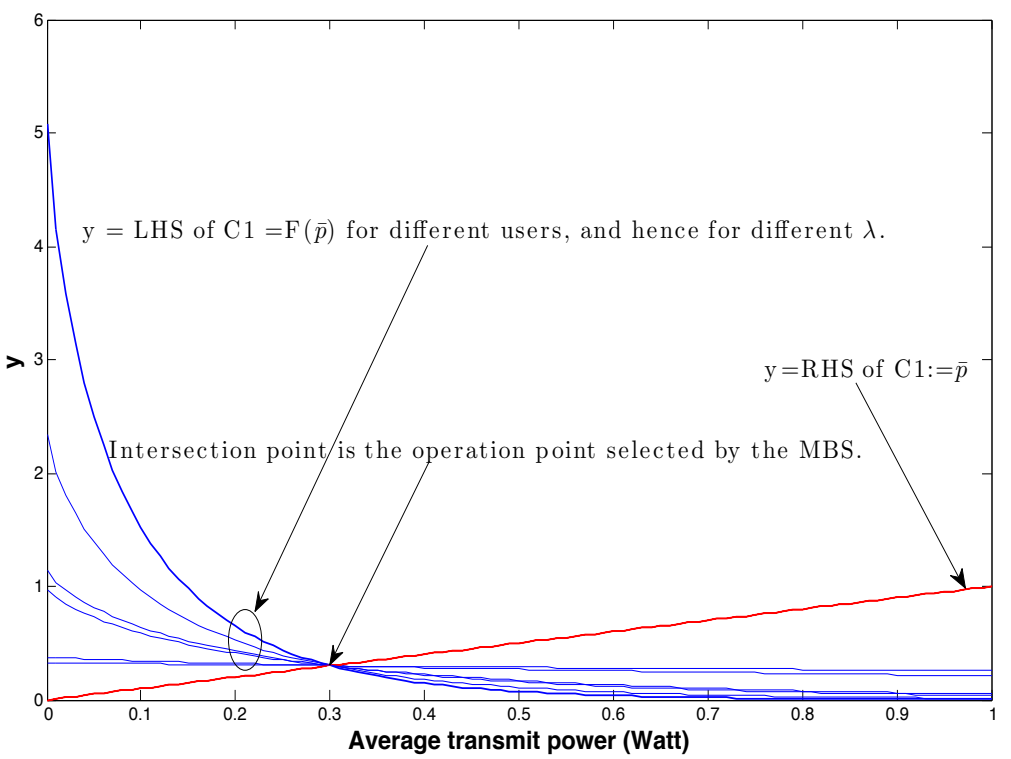

Figure 6.3: The point that realizes consistent condition $(C 1)$. The selected operating point here is: $\bar{p}_{m}=0.3$.

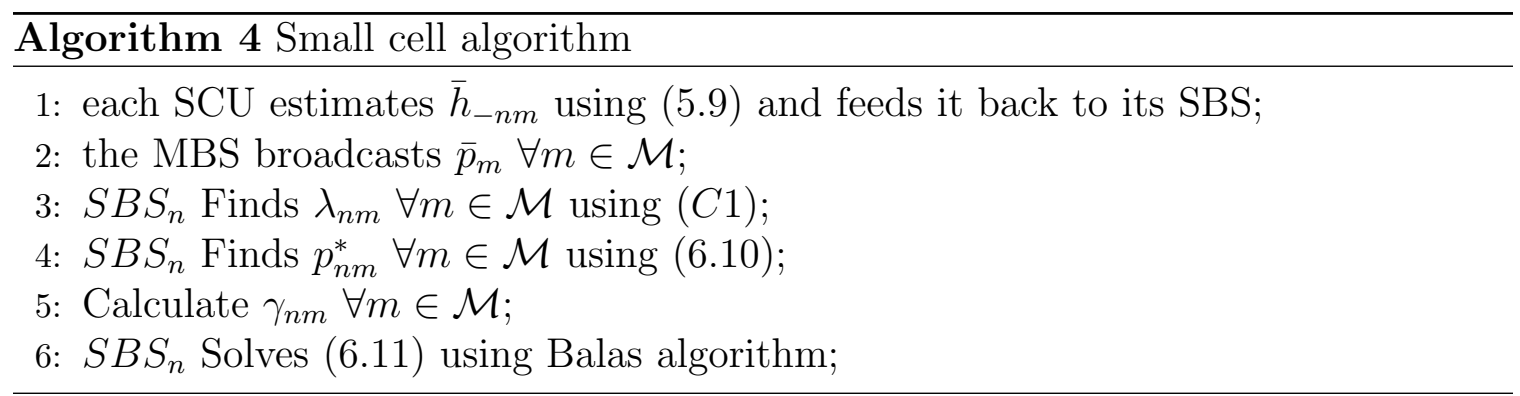


interference channels: $\bar{h}_{-j m}=\operatorname{mean}\left\{h_{j 1 m}, \ldots, h_{j n m}, \ldots, h_{j N m}\right\}$. This estimation can be simultaneously achieved similarly like each $S C U_{n}$ estimates $\bar{h}_{-n m}$, but without MBS transmitting. Thus,

$$
\bar{h}_{-j m} \approx \frac{p_{j m}^{(r)}-B N_{o}}{N p_{t}}
$$

where $p_{j m}^{(r)}$ is the received power at $M U_{j}$ during the training period, and $p_{t}$ is a predetermined value. Therefore, the MBS can anticipate the reaction of the SBSs by applying MFA:

$$
\begin{gathered}
I_{j m}=\sum_{k=1}^{N} p_{k m} h_{j k m} \approx N \bar{h}_{-j m} \bar{p}_{m} \\
\text { as } N \longrightarrow \infty
\end{gathered}
$$

Accordingly, the overlay problem can be written as:

$$
\begin{aligned}
& \underset{X_{j m}, p_{0 m}}{\operatorname{minimize}} \sum_{m \in \mathcal{M}} p_{0 m} \\
& \text { subject to } \\
& \sum_{m \in \mathcal{M}} B \log _{2}\left(1+\frac{p_{0 m} h_{j 0 m}}{\beta_{j} \sigma_{j m}^{2}}\right) X_{j m} \geq R_{j}, \forall j, \\
& \sum_{m \in \mathcal{M}} p_{0 m} \leq p_{\text {total }}^{(C)}, \\
& \sum_{j \in \mathcal{J}} X_{j m} \leq 1, \forall m, \\
& X_{j m} \in\{0,1\}, \forall m \& j, \\
& p_{0 m} \geq 0, \forall m,
\end{aligned}
$$

where $\sigma_{j m}^{2}=N \bar{h}_{-j m} \bar{p}_{m}+B N_{o}$ that is now known to the MBS, and hence this problem is indeed a single cell resource allocation problem. A variety of optimization techniques are available for the single cell resource allocation problem (see [61-63] and the references therein).

In this chapter, we also consider simplifying the above single cell problem, and 
putting it into a framework of a general algorithm. The optimization problem in (6.14) is a mixed integer non-linear programming (MINLP) whose complexity level is NP-complete [42]. However, this problem can be converted into a binary convex optimization by adding an auxiliary constraint and making slight changes in the notations as follows.

$$
\begin{aligned}
& \underset{X_{j m}, p_{j m}}{\operatorname{minimize}} \sum_{j=1}^{J} \sum_{m=1}^{M} p_{j m} \\
& \text { subject to } \\
& \sum_{m=1}^{M} B \log _{2}\left(1+\frac{p_{j m} h_{j 0 m}}{\beta_{j} \sigma_{j m}^{2}}\right) \geq R_{j}, \forall j, \\
& p_{j m}-p_{\text {total }}^{(C)} X_{j m} \leq 0, \forall m \& j,(\text { Aux. Const.) } \\
& \sum_{j=1}^{J} \sum_{m=1}^{M} p_{j m} \leq p_{\text {total }}^{(C)}, \\
& \sum_{j=1}^{J} X_{j m} \leq 1, \forall m, \\
& X_{j m} \in\{0,1\}, \forall m \& j, \\
& p_{j m} \geq 0, \forall m \& j,
\end{aligned}
$$

where $p_{j m}$ is the transmit power from the MBS to $M U_{j}$ (i.e., if subchannel $m$ is assigned to $M U_{j}$, then $p_{j m}=p_{0 m} \neq 0$ and $p_{i m}=0$ for all $\left.i \neq j\right)$. The purpose of the auxiliary constraint is to make sure that if $X_{j m}=0$, then $p_{j m}=0$ while if $X_{j m}=1$, then $p_{j m}>0$.

Problem (6.15), which is equivalent to the original problem, can be solved by applying the convex outer approximation (COA) algorithm [64]. This algorithm is readily implemented in Aimms software ${ }^{4}[65]$. In our simulations, we used Aimms in

\footnotetext{
${ }^{4}$ http://business.aimms.com/. Aimms is a popular software that is largely used by several commercial corporations.
} 
parallel with Matlab to solve the above problem.

\subsubsection{Overlay Interference Control Algorithm}

In this subsection, we summarize the overlay algorithm that runs in the MBS in order to control the cross-layer interference. First, the MBS would select an initial value of $\bar{p}_{m}$ for every subchannel; however, for simplicity, we consider it here equally likely over all subchannels. Then, the MBS would solve (6.15). If the problem is not feasible, then, it will reduce the value of $\bar{p}_{m}$ by a certain amount and try again. The MBS will keep iterating until reaching feasibility. Once it reaches feasibility, then the MBS broadcasts the value of $\bar{p}_{m}$ to all SBSs.

Algorithm 5 briefly describes the proposed overlay interference control scheme that runs in the MBS.

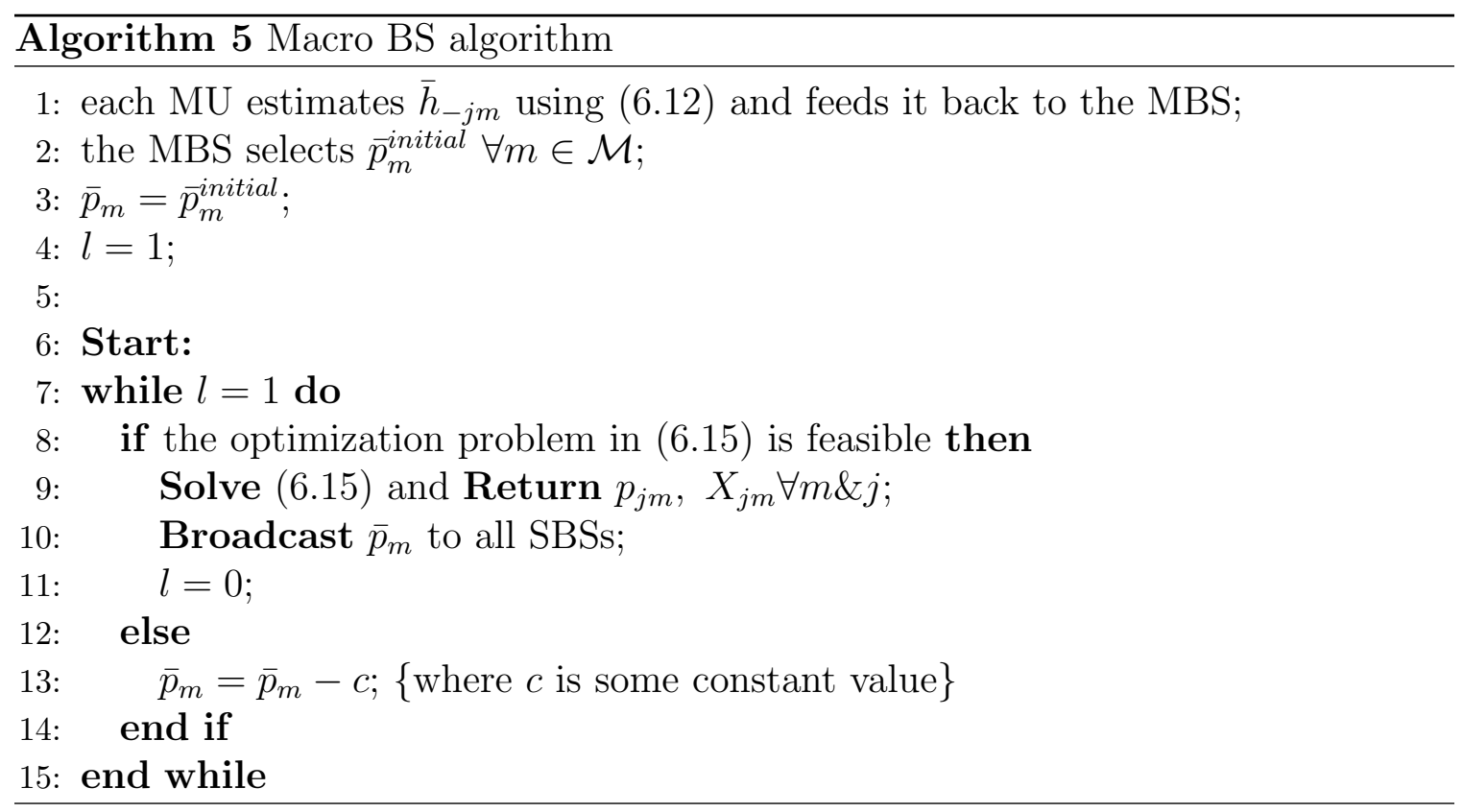




\subsubsection{Selecting $\bar{p}_{m}^{\text {initial }}$}

In case there are no MUs or very few of them resulting in some unused subchannels by the MBS, what would be the best value of $\bar{p}_{m} \forall m$ ? As it is noticeable from the current and previous sections, $\bar{p}_{m}$ is playing a crucial role, and it constitutes the degree of correlation between all players. The objective of this subsection is to find a method of selecting the value of $\bar{p}_{m}$ in case subchannel $m$ would not be used by the MBS, and also the initial value of $\bar{p}_{m}$ (i.e., $\bar{p}_{m}^{\text {initial }}$ ) in case $m$ is used by the MBS. Therefore, we will first investigate the impact of $\bar{p}_{m}$ on the utility function of SBSs in the absence of the MBS and MUs (i.e., $\sigma_{n m}^{2}=B N_{o}$ ).

Since $\bar{p}_{m}$ is the average of the transmit power across the interferers $(\forall i \in \mathcal{N} ; i \neq$ $\left.\left\{S B S_{n}\right\}\right)$, it looks that as $\bar{p}_{m}$ increases, the utility function of the interfered cell $n$ would decrease. In the following, we will show that this is not the case!

Theorem 3. In the absence of $M B S$, the utility function of $S B S_{n}$ monotonically increases with $\bar{p}_{m}$ as long as the effective transmit power policy is $p_{n m}^{*}$ in (6.10).

Proof. We start our proof by introducing the following definition.

Definition 5 (Best utility value). Let $u_{n m}^{*}\left(\bar{p}_{m}\right)$ be player $S B S_{n}$ 's utility as a function of $\bar{p}_{m}$, when player $S B S_{n}$ adopts the policy in (6.10). That is,

$$
u_{n m}^{*}\left(\bar{p}_{m}\right)=\alpha_{n} \ln \left(1+\frac{p_{n m}^{*} h_{n m}}{N^{\prime} \bar{h}_{-n m} \cdot \bar{p}_{m}+B N_{o}}\right)-\lambda_{n m} p_{n m}^{*} .
$$

Plugging the value of $p_{n m}^{*}$ into (6.16), this would translate into: 


$$
u_{n}^{*}\left(\bar{p}_{m}\right)=\left\{\begin{array}{cc}
\alpha_{n}\left(\ln \left(\frac{h_{n m}}{h_{n m}^{(l)}}\right)+\frac{h_{n m}^{(l)}}{h_{n m}}-1\right), & h_{n m} \geq h_{n m}^{(l)} \\
0 & \text { Otherwise }
\end{array}\right.
$$

where $h_{n m}^{(l)}=\frac{\lambda_{n m}}{\alpha_{n}}\left[N^{\prime} \bar{h}_{-n m} \cdot \bar{p}_{m}+B N_{o}\right]$.

In the following, we give a mathematical explanation of our claim that $u_{n m}^{*}$ increases with respect to $\bar{p}_{m}$. However before we move on, notice that $\lambda_{n m}$ is not a different degree of freedom, but rather it is a function of $\bar{p}_{m}$ through the condition $(C 1)$. Thus, in the subsequent paragraphes, $\frac{d \lambda_{n m}}{d \bar{p}_{m}}$ is denoted by $\lambda_{n m}^{\prime}$ that is negative amount owing to the inverse relation described by $(C 1)$ and depicted in Fig. 6.2.

Obviously, to examine the effect of adjusting $\bar{p}_{m}$ on the best utility value of a typical user, we should look at the derivative of $u^{*}$ with respect to $\bar{p}_{m}$.

$$
\frac{d u_{n m}^{*}}{d \bar{p}_{m}}= \begin{cases}\alpha_{n} h_{m}^{(l)^{\prime}}\left(\frac{1}{h_{n, n}}-\frac{1}{h_{n m}^{(l)}}\right), & h_{n m}>h_{n m}^{(l)} \\ 0 & h_{n m}<h_{n m}^{(l)},\end{cases}
$$

where

$$
h_{m}^{(l)^{\prime}}=\frac{d h_{n m}^{(l)}}{d \bar{p}_{m}}=\frac{\lambda_{n m}^{\prime}\left(N^{\prime} \bar{h}_{-n} \bar{p}_{m}+B N_{o}\right)+N^{\prime} \bar{h}_{-n} \lambda_{n m}}{\alpha_{n}} \leq 0 .
$$

The negativity of $h_{m}^{(l)^{\prime}}$ is due to the fact that $\lambda_{n m}^{\prime}<0$. Fig. (6.4) shows the decreasing relation between $h_{n m}^{(l)}$ and $\bar{p}_{m}$. In the subsequent, we will show that this inequality is, in fact, a strict inequality.

According to (6.18), if $h_{n m}>h_{n m}^{(l)}$, then $\frac{\partial u_{n}^{*}}{\partial \bar{p}_{m}} \geq 0$ and hence $u_{n}^{*}$ is a nondecreasing function with respect to $\bar{p}_{m}$. Now we would like to select $\bar{p}_{m}$ such that $u_{n}^{*}$ is maximized. According to Weierstrass theorem, it is observable that for $h_{n m} \geq h_{n m}^{(l)}, u_{n}^{*}$ attains an 


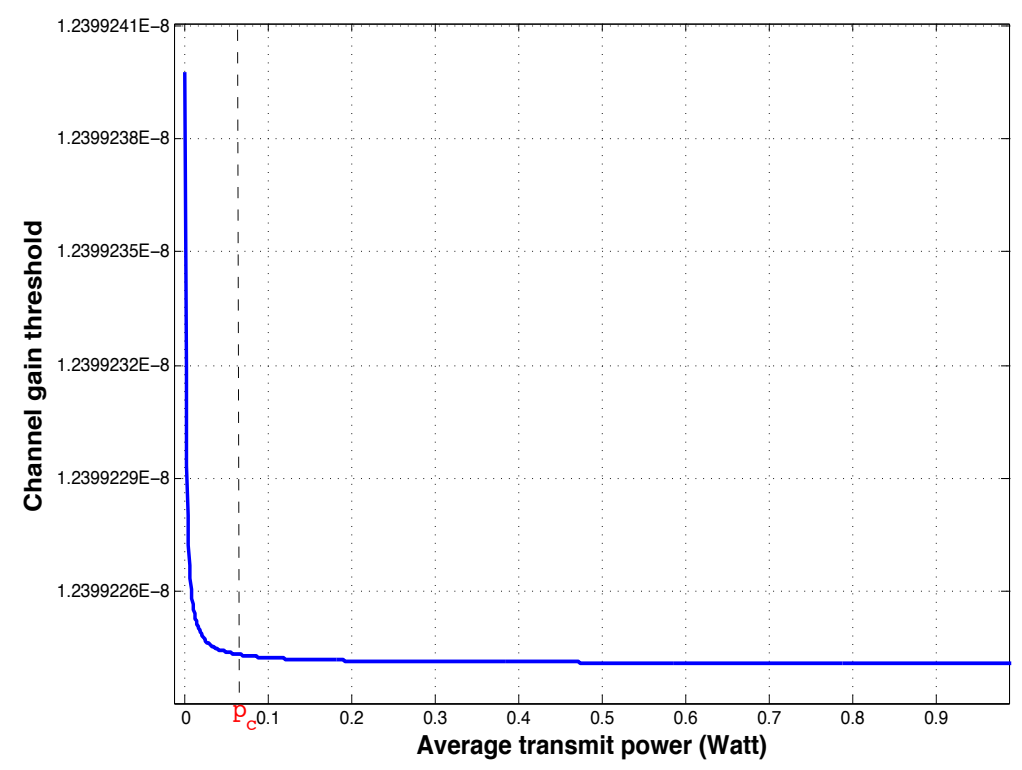

Figure 6.4: Cut-off channel gain $\left(h_{n m}^{(l)}\right)$ as a function of $\bar{p}_{m}$.

extremum.

A necessary condition for the existence of a maximizer $\bar{p}_{m}^{*}$ is $\frac{\partial u_{n}^{*}}{\partial \bar{p}_{m}}\left(\bar{p}_{m}^{*}\right)=0$. From (6.18), $\alpha_{n}$ and $\left(\frac{1}{h_{n m}}-\frac{1}{h_{n m}^{(l)}}\right)$ cannot be zero for $h_{n m}>h_{n m}^{(l)}$. Thus, the necessary condition for $u_{n}^{*}$ to attain a maximizer is reduced to $h_{m}^{(l)^{\prime}}=0$.

Interestingly, the problem of maximizing $u_{n}^{*}$ is equivalent to the problem of minimizing outage probability, that is,

$$
\begin{aligned}
\min _{\bar{p}_{m}} P_{\text {out }} & =\min _{\bar{p}_{m}} P\left(h_{n m} \leq h_{n m}^{(l)}\right) \\
& =\min _{\bar{p}_{m}}\left(1-e^{-\mu_{n} h_{n m}^{(l)}}\right) \equiv \min _{\bar{p}_{m}} h_{n m}^{(l)}
\end{aligned}
$$

Solving this problem implies $h_{m}^{(l)^{\prime}}=0$. Therefore,

$$
h_{m}^{(l)^{\prime}}=0 \Longrightarrow-\lambda_{n m}^{\prime}=\frac{\lambda_{n m}^{2} N^{\prime} \bar{h}_{-n}}{\alpha_{n} h_{n m}^{(l)}}
$$

\section{Condition $(C 1)$ revisited:}


The consistent condition $(C 1)$ could be written as:

$$
e^{-\mu_{n} h_{n m}^{(l)}}-\mu_{n} h_{n m}^{(l)} \cdot \mathrm{E}_{1}\left(\mu_{n} h_{n m}^{(l)}\right)=\frac{\lambda_{n m} \bar{p}_{m}}{\alpha_{n}}
$$

Differentiating both sides of condition $(C 1 . a)$ with respect to $\bar{p}$ yields $(6.21)$.

$$
-\mu_{n} h_{m}^{(l)^{\prime}} e^{-\mu_{n} h_{m}^{(l)}}-\left[\mu_{n} h_{m}^{(l)^{\prime}} \cdot \mathrm{E}_{1}\left(\mu_{n} h_{m}^{(l)}\right)+\mu_{n} h_{m}^{(l)} \cdot \frac{d\left(\mathrm{E}_{1}\left(\mu_{n} h_{m}^{(l)}\right)\right)}{d \bar{p}_{m}}\right]=\frac{\lambda_{n m}+\lambda_{n m}^{\prime} \bar{p}_{m}}{\alpha_{n}} .
$$

Identity (5.1.26) in [66] implies that $\frac{d\left(\mathrm{E}_{1}\left(\mu_{n} h_{m}^{(l)}\right)\right)}{d \bar{p}_{m}}=-\mathrm{E}_{0}\left(\mu_{n} h_{m}^{(l)}\right) \cdot \mu_{n} h_{m}^{(l)^{\prime}}$. Thus,

$$
-\mu_{n} h_{m}^{(l)^{\prime}} e^{-\mu_{n} h_{m}^{(l)}}-\mu_{n} h_{m}^{(l)^{\prime}} \cdot \mathrm{E}_{1}\left(\mu_{n} h_{m}^{(l)}\right)+\mu_{n} h_{m}^{(l)} \cdot \mathrm{E}_{0}\left(\mu_{n} h_{m}^{(l)}\right) \cdot \mu_{n} h_{m}^{(l)^{\prime}}=\frac{\lambda_{n m}+\lambda_{n m}^{\prime} \bar{p}_{m}}{\alpha_{n}}
$$

Furthermore, according to identity (5.1.24) in [66], $\mathrm{E}_{0}\left(\mu_{n} h_{m}^{(l)}\right)=\frac{e^{-\mu_{n} h_{m}^{(l)}}}{\mu_{n} h_{m}^{(l)}}$. Therefore, (6.22) could be written as,

$$
-\mu_{n} h_{m}^{(l)^{\prime}} \cdot \mathrm{E}_{1}\left(\mu_{n} h_{n m}^{(l)}\right)=\frac{\lambda_{n m}+\lambda_{n m}^{\prime} \bar{p}_{m}}{\alpha_{n}}
$$

Since we are interested in the point where $h^{(l)^{\prime}}=0,(6.23)$ yields,

$$
-\lambda_{n m}^{\prime}=\frac{\lambda_{n m}}{\bar{p}_{m}}
$$

Thus, from (6.20) and (6.24), we have:

$$
\frac{\lambda_{n m}^{2} N^{\prime} \bar{h}_{-n}}{\alpha_{n} h_{n m}^{(l)}}=\frac{\lambda_{n m}}{\bar{p}_{m}}
$$

Rearranging (6.25) and substituting the value of $h_{n m}^{(l)}$ yields,

$$
N^{\prime} \bar{h}_{-n} \bar{p}_{m}=N^{\prime} \bar{h}_{-n} \bar{p}_{m}+B N_{o}
$$


As we can see from (6.26), there is contradiction due to the fact that noise power is always greater than zero. Therefore, the necessary condition $h_{m}^{(l)^{\prime}}=0$ failed (i.e., $h_{m}^{(l)^{\prime}}$ cannot equal to zero at the first place), which in turn negates the existence of the maximizer within any open interval (or equivalently the minimizer for the outage probability). Therefore, $u_{n m}^{*}$ monotonically increases w.r.t. $\bar{p}_{m}$ as shown in Fig. (6.5), and hence $\frac{\partial u_{n m}^{*}}{\partial \bar{p}_{m}}>0$. Moreover, the extremum value occurs at the end point $p_{m}^{\text {max }}$.

It is worth noting that the above result goes coherently with the results in [67] and [41]. Fig. 6.5 shows $u^{*}$ as a function of $\bar{p}_{m}$. The increasing regime in Fig. 6.5 is essentially due to the relative strength of the desired channel compared to the interfering channel. If all SBSs transmit with power levels where $\bar{p}_{m}<p_{c}$, then the received interference would be very weak. Consequently, due to the relative strength of the desired channel of each player, there's a chance for increasing the utility value by increasing the transmit power, without incurring too much received interference. This region $\left(\bar{p}_{m}<p_{c}\right)$ may be called the desired channel dominant regime. Once $\bar{p}_{m}=p_{c}$, the interference effect kicks in and almost rules out the gain of any increase in $\bar{p}_{m}$. This region $\left(\bar{p}_{m} \geq p_{c}\right)$ may be called the interference dominant regime.

In our scheme, the best utility value is maintained in increasing mode w.r.t. $\bar{p}_{m}$ as shown in Fig. 6.5. What protects it? In most of the other schemes, $\lambda_{n m}$ is chosen arbitrarily. In this proposed scheme, $\lambda_{n m}$ is a function of $\bar{p}_{m}$ via $(C 1)$. Therefore, once $\bar{p}_{m}$ enters the interference dominant regime, $(C 1)$ is going to prevent the negative impacts of this regime by adjusting the price factor $\lambda_{n m}$ of every player. In other words, this technique would regulate the chain reaction.

Due to the continuous improvement in $u_{n m}^{*}$ with respect to $\bar{p}_{m}$, one may choose $\bar{p}_{m}$ to be the highest possible value that in turn maximizes the utility function. However, this positive effect on utility function due to increasing $\bar{p}_{m}$ is far outweighed by a negative effect on energy efficiency. If $\bar{p}_{m}>p_{c}$, the ratio of utility gain to power 


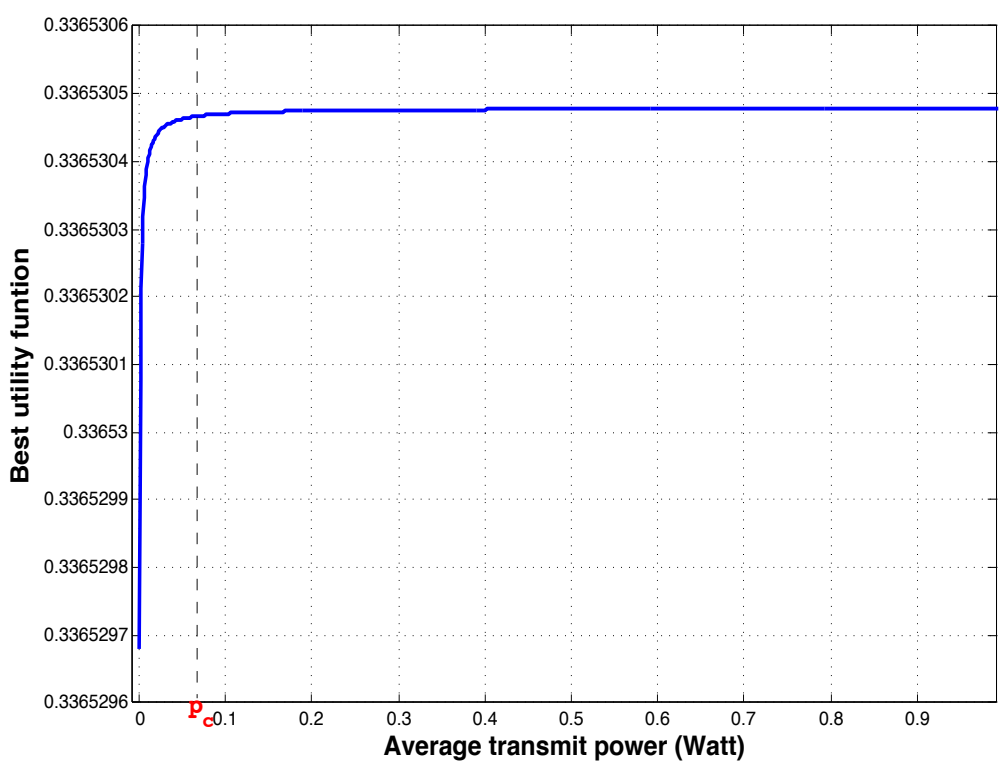

Figure 6.5: The best utility value $\left(u^{*}\right)$ as a function of $\bar{p}_{m}$, where $h_{n m}>h_{n m}^{(l)}$.

spending is negligible. Thus, for best trade-off between the spectral efficiency and energy efficiency, we suggest the initial value of $\bar{p}_{m}$ to be:

$$
\bar{p}_{m}^{\text {initial }}=\max _{n \in \mathcal{N}}\left\{p_{c_{n}}\right\}
$$

We simulated a network in the absence of MBS. Figs. (6.6) and (6.7) clearly depict how the energy efficiency is more sensitive to $\bar{p}$ than the spectral efficiency (SE). Although $\bar{p}$ is different in the 3 forms of the MFG-based system, their spectral efficiencies are almost identical. This phenomenon is well explained using Fig. 6.5. As depicted in Fig. 6.5 , if $\bar{p}>p_{c}$, the utility value would be almost indifferent to changes in average transmit power $\bar{p}$. However, the clear contrast in the performance of these 3 forms of MFG system appears in the energy efficiency evaluation. It is obvious that the lower the $\bar{p}$ is, the better the energy efficiency. Fig. (6.8) also shows that the border user SE exhibits similar behaviour as that of the aggregate SE.

The value of $p_{c_{n}}$ depends on the other parameters (i.e., $N, h_{n m}, \bar{h}_{-n m}$ ). For example, if $N$ is high, then the interference impact on each receiver will be in effect early 


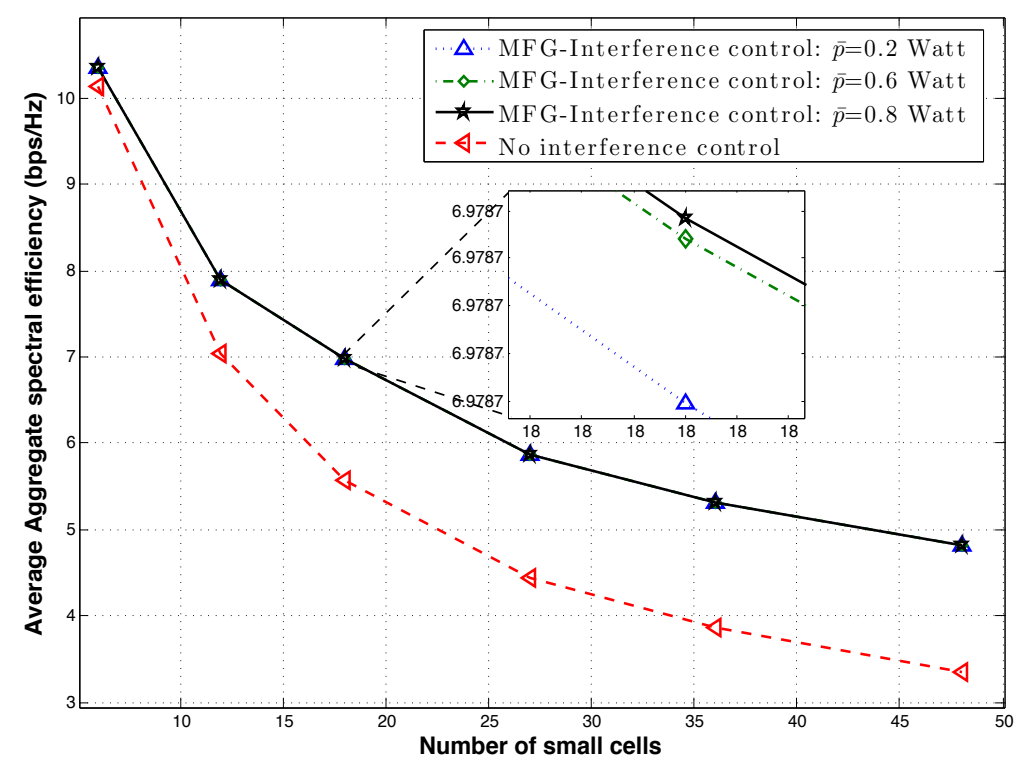

Figure 6.6: Average aggregate spectral efficiency vs. the number of small cells.

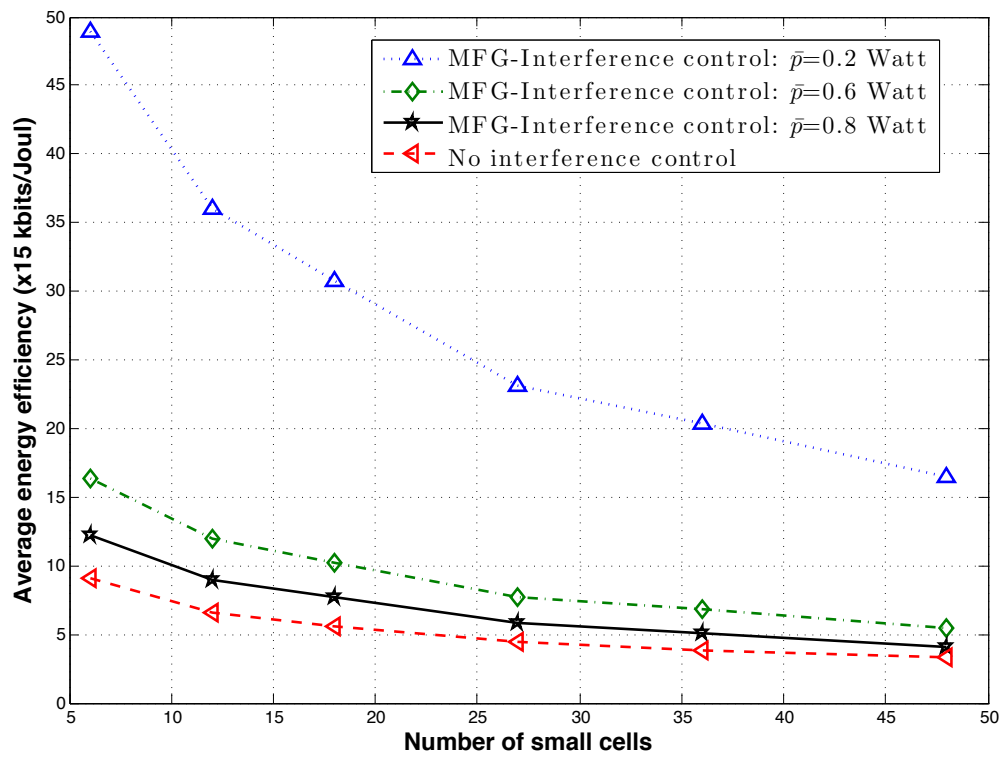

Figure 6.7: Average energy efficiency vs. the number of small cells. 


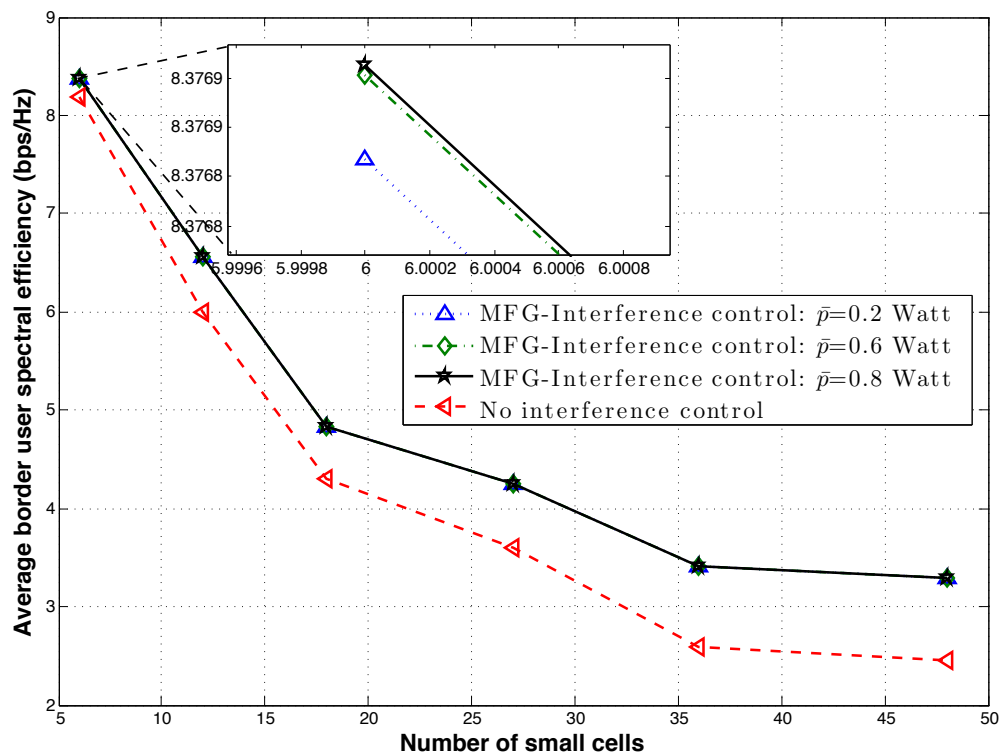

Figure 6.8: Average border user spectral efficiency vs. the number of small cells.

(i.e., $p_{c_{n}}$ would be relatively low), which would also be the case if $\bar{h}_{-n m}$ is strong or $h_{n m}$ is weak. On the other hand, if $N$ is a small value, say 1 , then $p_{c_{n}}$ would be the maximum possible transmit power since the interference dominant regime would not exist as $N-1=0$. Finally, it is very probable that the values of $\bar{p}_{m}$ for each $m \in \mathcal{M}$ are in the same order. Thus, for simplicity, the initial value of $\bar{p}_{m}$ in Algorithm 5 could be chosen as the same value for all $m \in \mathcal{M}$.

\subsection{Simulation Results and Discussions}

The goal of this section is to evaluate the performance of the proposed algorithms. We simulated an area $A$ consisting of an MBS with its associated users, as well as several small BSs with their users' SCUs. The simulation is based on the system model described in Section 6.1 and system parameters shown in Table 6.1. The simulation has been performed twice for two different deployments. In the first deployment, operators are assumed to carefully select the locations of the SBSs. Examples of this kind of deployment include pico cells and small cells within a large enterprise. The second 
Table 6.1: Simulation parameters

\begin{tabular}{|c|c|}
\hline Parameters & Value \\
\hline \hline System frequency: $f_{c}$ & $2000 \mathrm{MHz}$ \\
\hline System bandwidth & $6 \mathrm{MHz}$ \\
\hline No. of data subchannels & 300 \\
\hline$p_{\text {total }}^{(C)} p_{\text {total }}^{(F)}$ & $46 \mathrm{dBm}, 30 \mathrm{dBm}$ (respectively) \\
\hline Small cell radius $r_{F}$ & $30 \mathrm{~m}$ \\
\hline No. of MUs & 5 \\
\hline MUEs required rate: $R_{j}$ & $0.5 \mathrm{Mbps}$ \\
\hline Noise power spectral density & $-178 \mathrm{dBm} / \mathrm{Hz}$ \\
\hline Shadow fading deviation & $8 \mathrm{~dB}$ \\
\hline Small scale fading & Rayleigh fading \\
\hline
\end{tabular}

simulation assumes that the deployment is random where end users in a residential area install their own SBSs. Moreover, to ensure that sufficient data is collected, we repeated the simulation for 40 replications. A general simulation setup is described in Appendix A.

In order to solve the mixed integer convex programming in (6.15), AIMMS software has been used along with MATLAB.

\subsubsection{Planned Deployment}

In a hexagonal topology, five different systems were simulated under the same basic conditions except that they use different interference control schemes. They are as follows:

- The first system is equipped with our proposed mean-field game-based interference control (MFG) in the small cells level. The optimal channel and power assignment in the macrocell level is accomplished by solving (6.15) using COAbased algorithm [64]. We refer to this system by Opt\&MFG. 
- The second system is equipped with a conventional game theoretic interference control scheme. Since, in this case, MBS cannot estimate the interference from small cells, the MUs channel assignment is performed using Hungarian algorithm on the matrix of desired channels. Then, a conventional game is to be played over each subchannel by all BSs including MBS. The action of each player is the transmit power. If $m^{\text {th }}$ subchannel was assigned to $j^{\text {th }} \mathrm{MU}$, the utility function of MBS is $-\left(\gamma_{j m}-\Gamma_{j m}\right)^{2}$, where $\Gamma_{j m}$ is the target sinr required by $M U_{j}$ over $m^{t h}$ subchannel. On the other hand, the utility function of each SBS is $u_{n m}=\alpha_{n} \ln \left(1+\frac{\gamma_{n m}}{\beta_{n m}}\right)-\lambda_{n m} p_{n m}$. We refer to this system by H\&CG.

- The third system is equipped with an interference control scheme that has been designed to solely control the cross-layer interference, and assuming free colayer interference [2]. While the utility function of MBS is as same as that in the second system, the utility function of the SBSs is given by $1-e^{-a_{n}\left(\gamma_{n m}-\Gamma_{n m}\right)}-$ $b_{n} p_{n m} h_{0 m}$, where $a_{n}$ and $b_{n}$ are operation parameters [2]. Here again the channel assignment in the macrocell level is performed using Hungarian algorithm on the matrix of desired channels. We refer to this system by H\&CL. Since this system has been designed based on the assumption of zero co-layer interference, we show its results in both cases: with and without co-layer interference effects. It has been said that co-layer interference can be made zero or closer to zero by borrowing a traditional solution from cellular network literature. Therefore, the following two frequency planning-based solutions are introduced.

- The fourth system controls the intercell interference by the traditional soft frequency reuse (SFR). The central users can access the whole granted spectrum with partial power whereas the border user can access only one of three equal portions of the spectrum. These three portions are orthogonal on adjacent cells. 
The ratio of the central users transmit powers to the border users transmit powers is $1 / 4[22]$.

- The fifth system adopts the traditional frequency reuse of factor 3 (Reuse-3). In Reuse-3, the granted spectrum is divided into 3 sub-bands, and each SBS is assigned a sub-band that is orthogonal to the allocated sub-bands in the adjacent SBSs.

For a given area, when a network is densified, there will be a simultaneous gain and loss. The gain is due to spatial frequency reuse as the number of BSs increases whereas the loss is due to the increased inter-cell interference at every receiver. Area spectral efficiency in bps/Hz per square kilometre is a good metric to capture the overall performance. Fig. 6.9 shows the area spectral efficiency (ASE) of all five schemes. Our proposed scheme slightly outperforms the H\&CG since the channels in the macrocell level are optimally assigned by solving (6.15), and hence the MBS transmit power is minimized. On the other hand, the Hungarian assignment is suboptimal. Thus the MBS transmit power achieved by the conventional game is not necessarily minimized. H\&CL systems is very competing; however, when the co-layer interference is included in the SINR calculation, its performance deteriorates due to the fact that the co-layer interference is not part of the design.

The main purpose of intercell interference management is to guard the performance of border users. Fig. 6.10 demonstrates the spectral efficiency of the border user in all systems. In case of free co-layer interference ${ }^{5}, \mathrm{H} \& \mathrm{CL}$ has the best border user SE. Our proposed scheme and H\&CG are quite comparable especially as the network becomes hyper-denser. Reuse-3 is known to be very effective in alleviating intercell interference on border users. However, that comes out of a weakened aggregate spectral efficiency. SFR was proposed essentially to improve the aggregate

\footnotetext{
${ }^{5}$ This is not a realistic assumption as we explained in the introduction.
} 


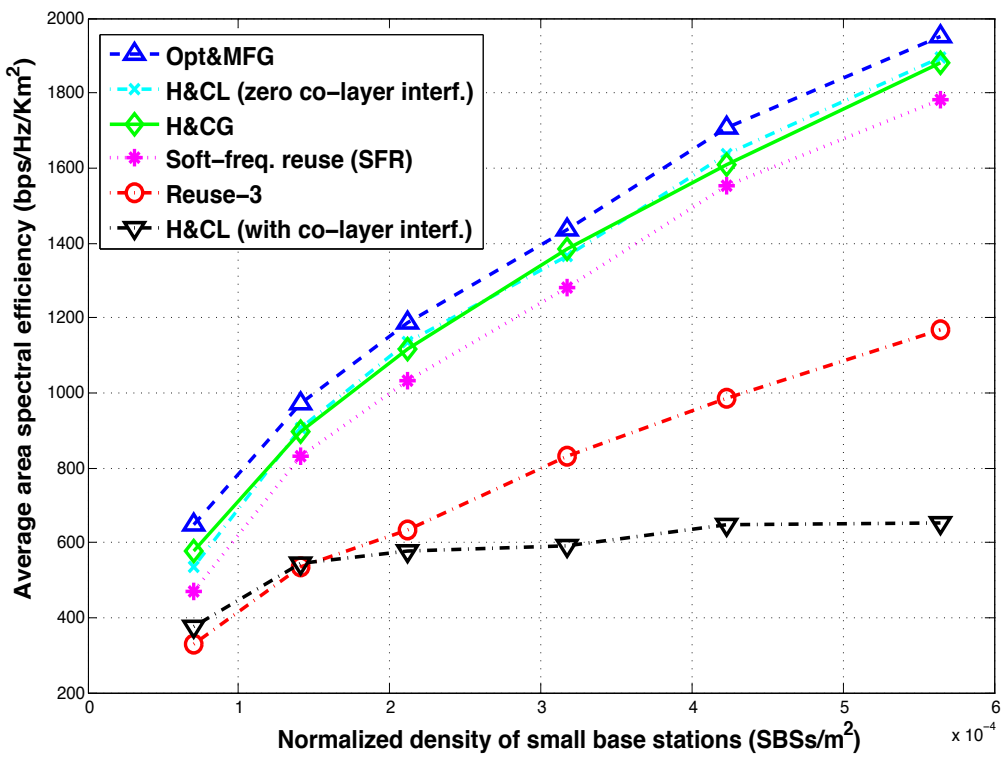

Figure 6.9: Average area spectral efficiency vs. normalized density of small cell base stations.

spectral efficiency of Reuse-3 (as shown in Fig. 6.9). However, the border users in SFR would experience relatively higher interference than in Reuse-3 as shown in 6.10. Unlike the first three schemes which deal with interference in an adaptive manner, Reuse-3 and SFR are fixed schemes.

Energy efficiency comparison is demonstrated in Fig. 6.11. The energy efficiency of the proposed scheme shows a decreasing pattern as the number of SBSs increases. This indicates that every time the network was densified, the MBS could still manage to serve its users by increasing its transmit power and without having to decrease the value of $\bar{p}$; otherwise, we could have seen an increasing pattern in EE w.r.t. the number of SBSs (we have shown at the end of Subsection 6.3.2 that EE increases when $\bar{p}$ is decreased). Thus, If the network is densified while the MBS does not have to decrease $\bar{p}$, then the cross-layer interference from MBS to SCU will increase causing a decreasing pattern in EE. H\&CL shows a good energy efficiency because according to this algorithm, SBSs would keep reducing its transmit power until cross layer interference at macrocell level becomes low enough for satisfying 


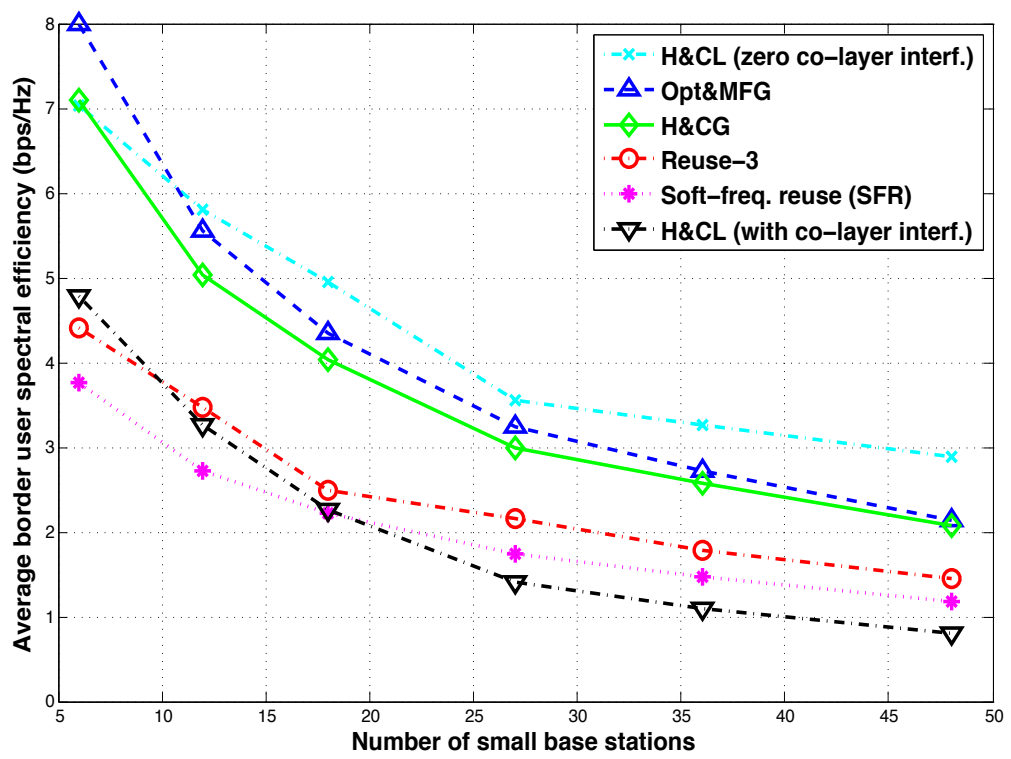

Figure 6.10: Average border user spectral efficiency vs. the number of small cell base stations.

MUs. The other version of this scheme has much lower energy efficiency due to the effect of the co-layer interference that is not being controlled. Reuse-3 has the lowest performance because each SBS has access to only one third of the spectrum.

\subsubsection{Random Deployment}

In random topology, since inter-cell coordination in dense closed access SBSs is prohibitive, it is very hard to apply any frequency coordination. Therefore, we will confine the discussion to the first 3 systems discussed earlier. To have a statistical view of the results, in this subsection we show the empirical cumulative density function $(\mathrm{CDF})$ of different metrics.

Fig. 6.12 shows the area spectral efficiency CDF. Our proposed scheme and H\&CG scheme behave almost similarly since our proposed scheme is just an approximation of H\&CG enhanced by an optimal channel and power assignment in the macrocell level. Assuming free co-layer interference, $\% 50$ of the time H\&CL scheme outperforms 


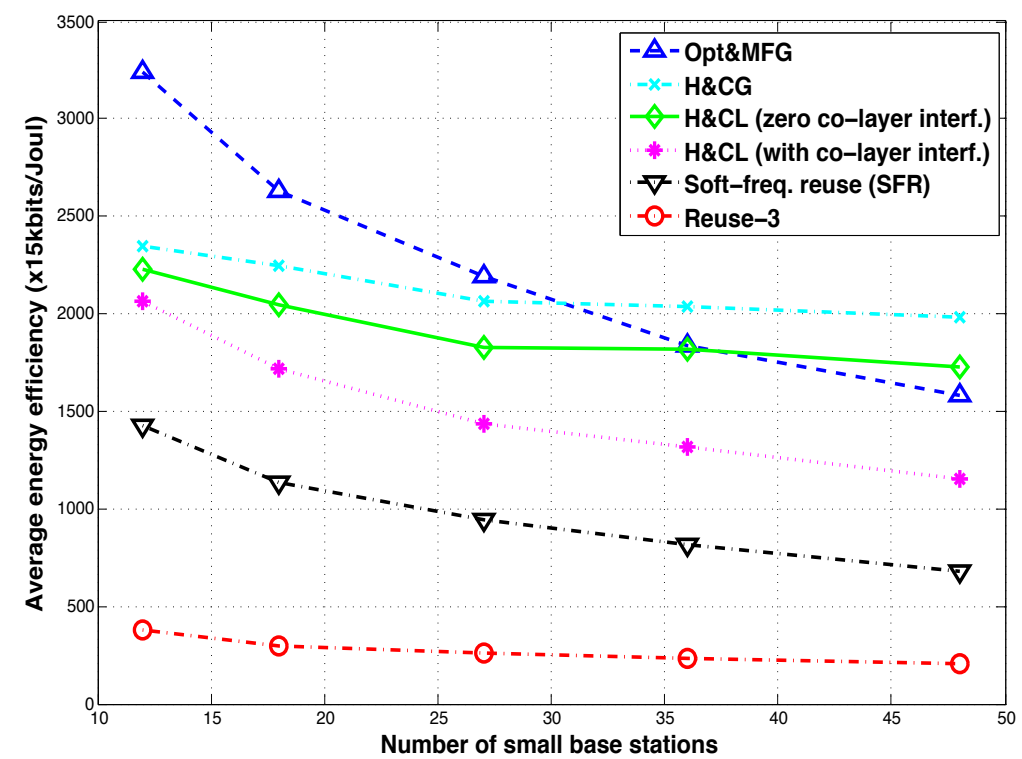

Figure 6.11: Average energy efficiency vs. the number of small cell base stations.

our proposed scheme and H\&CG. However, when co-layer interference is actually included in the SINR, the performance of H\&CL scheme deteriorates due to a lack of co-layer interference management. Fig. 6.13 shows that the H\&CL perform very well in serving border user as long as the co-layer interference is zero. Furthermore, the empirical CDFs of the border user spectral efficiency in H\&CG and Opt\&MFG almost matches due to the approximation relationship between them. However, the merit of our proposed scheme (Opt\&MFG) is that it is less complex than H\&CG. The transmit power in each small cell can simply be found via (6.10) (i.e., iterations aren't required). The only requirement is a simple estimation of $h_{-n m}^{-}$. On the other hand, Fig. 6.14 shows the CDF of the number of iterations required for game convergence in H\&CG where its average is 162 iterations. Finally, Fig. 6.15 shows the CDF of the MBS total transmit power required to satisfy the MUs QoS. The CDF related to Opt\&MFG shows a relatively higher variance compared to the other schemes. This is because in Algorithm 5, $\bar{p}$ is reduced in a discrete fashion by amount of $c$ Watts, which in turn reduces the expected interference at MUs in the same manner. Thus, 


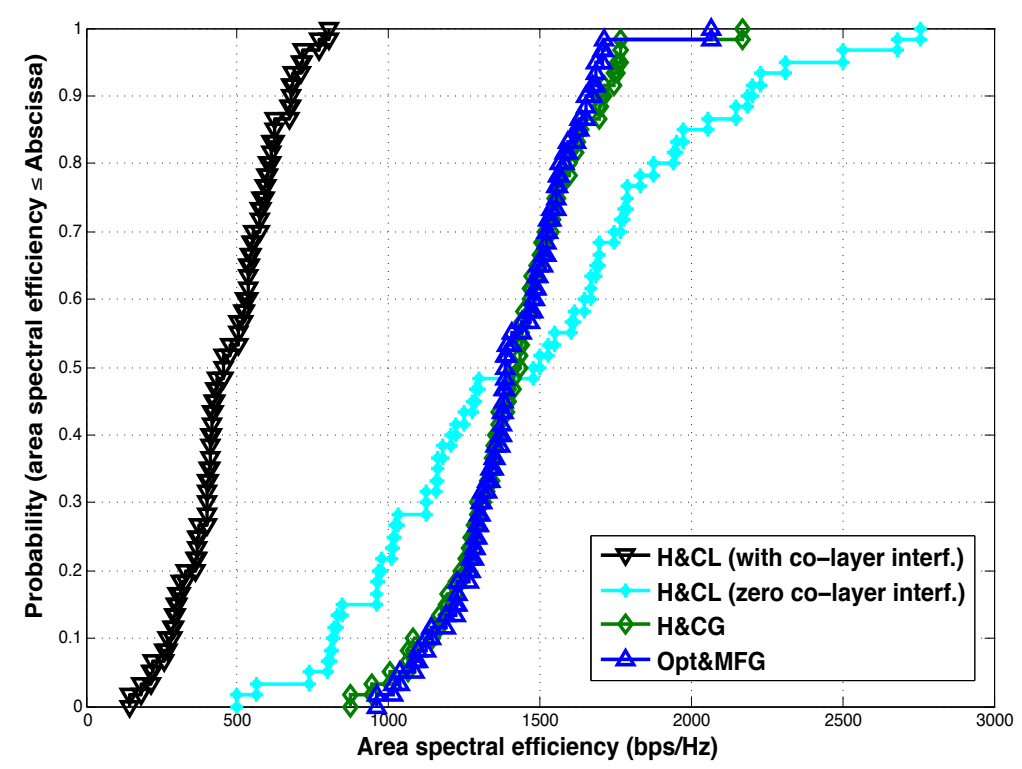

Figure 6.12: Empirical CDF of the area spectral efficiency in a random deployed network (The average number of SBSs is 27).

the required transmit power at macrocell will vary.

\subsection{Conclusions}

Considering a dense network of a large number of small cells, we proposed an interference control scheme that has two-levels of algorithms. The first is an overlay algorithm in which the MBS is modelled as the leader and the set of all SBSs is modelled as the follower. The second algorithm is based on a non-cooperative game in which the SBSs are considered the players. While the former algorithm is to control the cross-layer interference, the latter is to mitigate the co-layer interference. Using recent advances in mean field game theory, we decoupled the complex system into localized optimization problems. Each base station can implement its policy by using only its local information and some macroscopic information. In different network topologies, simulation results demonstrated that the proposed scheme achieves a competitive spectral efficiency as well as a good energy efficiency. 


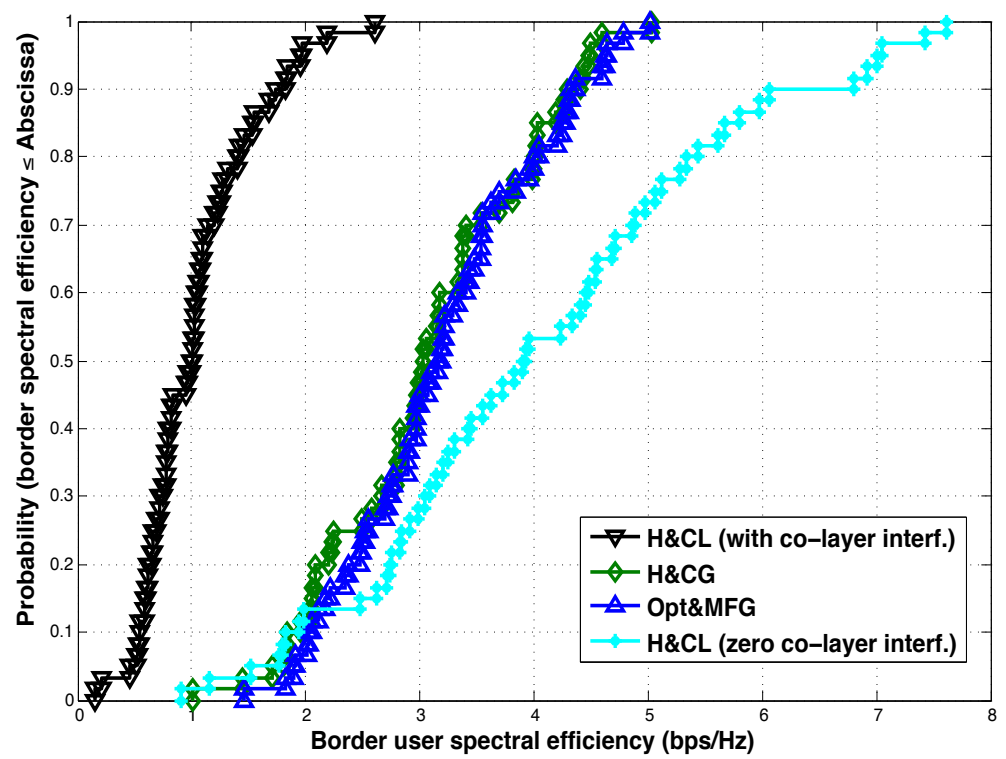

Figure 6.13: Empirical CDF of the border user spectral efficiency in a random deployed network (The average number of SBSs is 27).

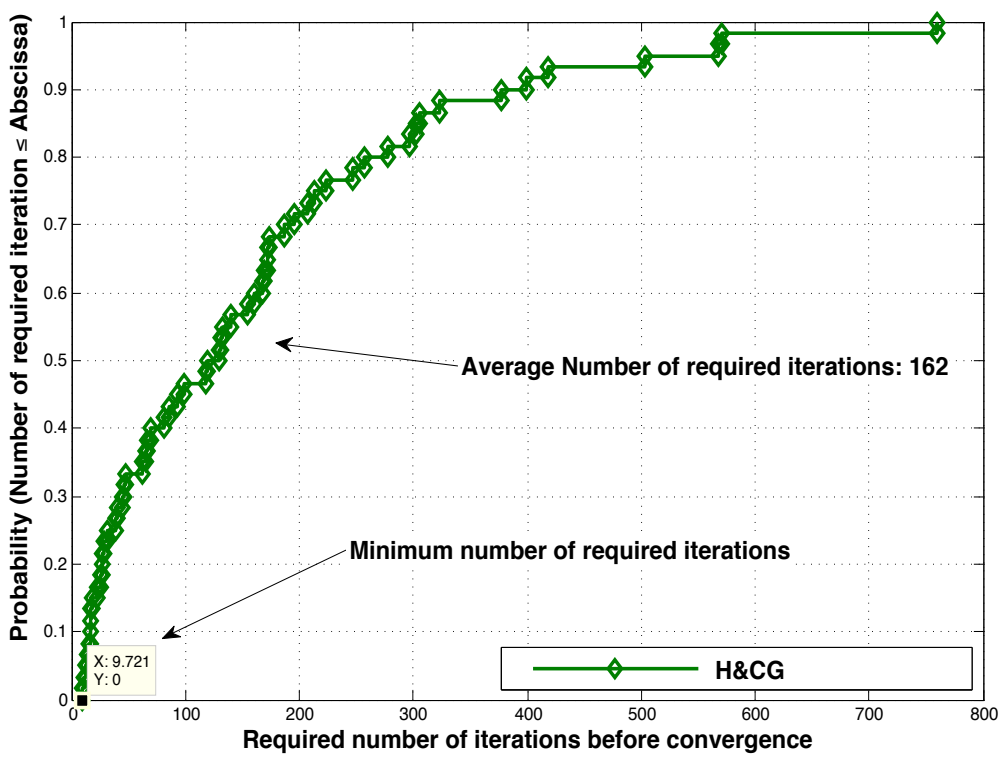

Figure 6.14: Empirical CDF of the required iterations for conventional game to converge (The average number of SBSs is 27). 


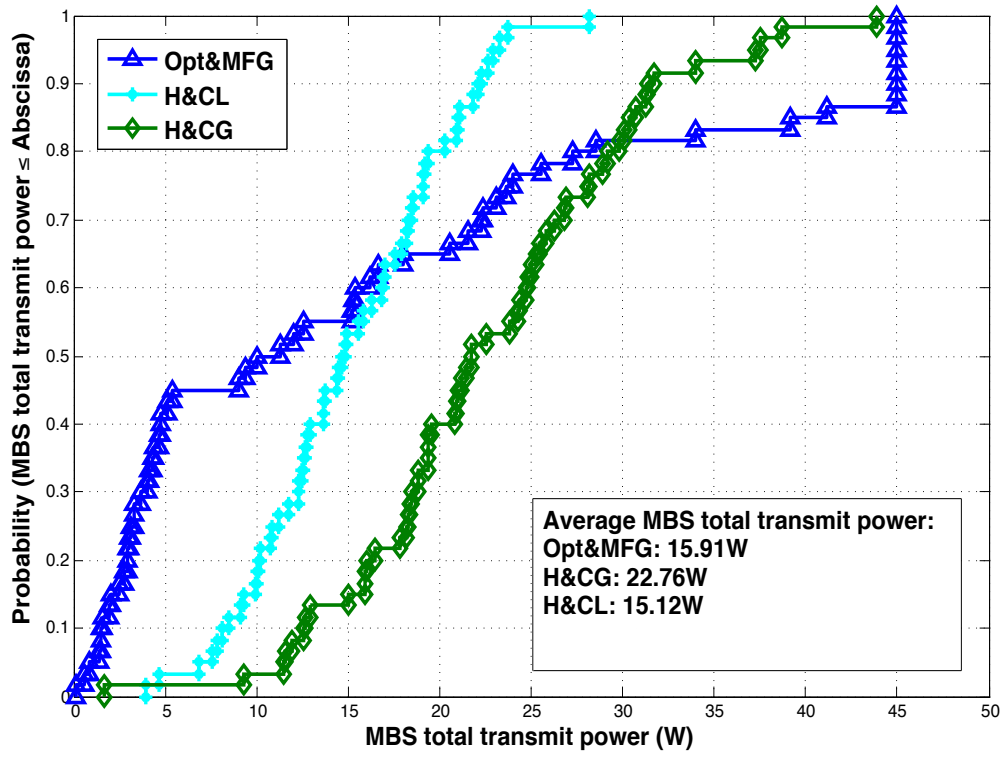

Figure 6.15: Empirical CDF of the total transmit power of MBS. 


\section{Chapter 7}

\section{Enhancing Cell Edge Users Performance in Open Access HetNets}

As mentioned in the introduction of this thesis, there are two types of HetNets: open access, in which any mobile user can access any open SBS, and closed access, where only those pre-registered users can access a given closed SBS [4]. In this chapter, the open access small cells HetNets will be considered.

Inter-cell interference is obviously the source of the network efficiency bottleneck, especially in cell edge. Traditionally, the channel of cell edge users is classified as an interference channel. The capacity of the interference channel has been an open research problem for a long time [70-72]. Researchers frequently obtain a higher achievable rate than ever before for such a channel, but its capacity has never been found.

Since cell edge users are the most common victims of inter-cell interference, coordinated multipoint (COMP) has been proposed to improve cell edge user data rates [73]. However, COMP requires a tight inter-BS coordination and a large amount of infor-

mation exchange. Moreover, it suffers from the outdated and noisy exchange of information [54].

In this chapter, we exploit the proximity of the nodes in the small cells network 
to create a less complex class of scheduling cell edge users. In a decentralized fashion, all SBSs solve a common problem to arrive at a unified optimal decision, and hence take more harmonious actions.

In conventional multiuser diversity, a base station transmitter schedules one user to maximize the data rate over the assigned channel. Thus, any given user might be scheduled or not. Unlike the TDMA scheduling scheme, which guarantees fairness at the expense of the network sum rate, this plain multiuser diversity achieves the maximum sum rate, at the users expense. For this reason, a fairness mechanism is sometimes imposed to this kind of diversity in order to guarantee a minimum level of fairness.

Given two cell edge users, as shown in Fig. 7.1, the conventional way of transmitting would result in a quantity of interference that degrades both users' rates, and in turn their achievable sum rate ${ }^{1}$. The packets of both users would suffer large delays due to the low service rate. When looking carefully at the problem, there are in fact 4 diverse ways of serving border users with respect to the SBSs. Thus, we take advantage of this diversity to enhance the achievable rate of the user equipment (UE) located at the cells edge. This increased multiuser diversity in scheduling does not only grow with the number of users but also with the number of SBSs involved. By adapting to the set of channels involved, the increased degree of freedom in scheduling will certainly enhance the achievable sum rate. However, there might be cases where both SBSs keep serving one user for a long time, causing an overflow on the queue of the other user.

In this chapter, we address different classes of scheduling to create a balance between the system-centric objective and the user-centric objective. The discussion is started with a simple model, and is then extended to a more general and realistic model. Extensive simulation results are presented to show the effectiveness of the

\footnotetext{
${ }^{1}$ Maximizing the sum rate is an important objective from the network viewpoint.
} 


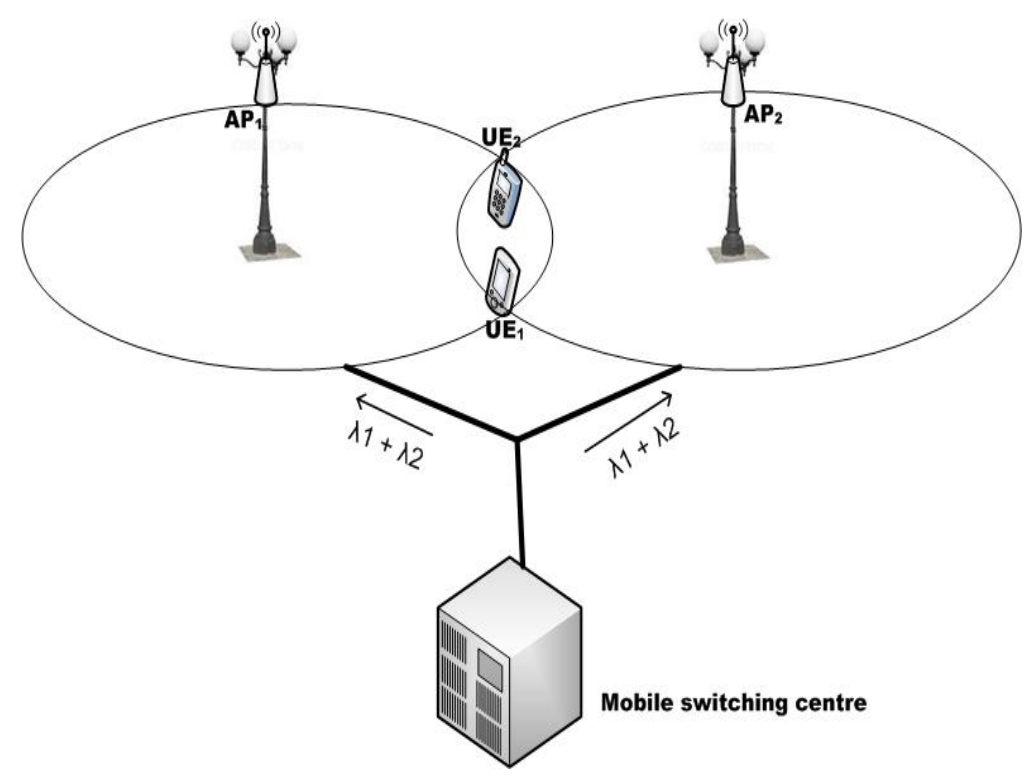

Figure 7.1: The problem of cell edge users in an open access small cells network.

proposed schemes.

The rest of this chapter is organized as follows. The system model is described in Section 7.1. A class of different schemes of scheduling decision making are introduced in Section 7.2, followed by a generalization of this formulation in Section 7.3. Then, various results of an extensive simulation are introduced in Section 7.4. Finally, this chapter is concluded in Section 7.5.

\subsection{System Description}

We consider the downlink problem of cell edge users in an open access small cells network. In a given busy area, where small SBSs are installed at many points, user equipments (UEs) are likely to be in an overlapping area between two (or more) SBSs (as shown in Fig. 7.1). Since cell edge users are relatively nearby both SBSs, the channel from each SBSs can be estimated by every cell edge user. This information is then broadcasted via the control channel. Due to the relative high power of the control channel, the channels state information is very likely to be correctly detected 
Table 7.1: Different scenarios for serving 2 cell edge users via 2 SBSs.

\begin{tabular}{|c|c|c|c|c|}
\hline $\mathrm{X}$ & Association & SINR & Achievable rate & $\begin{array}{l}\text { Channel } \\
\text { type }\end{array}$ \\
\hline 1 & $\begin{array}{l}S B S_{1} \rightarrow U E_{1} \\
S B S_{2} \rightarrow U E_{2}\end{array}$ & $\gamma_{1}=\frac{p_{1} g_{1,1}}{p_{2} g_{2,1}+\sigma_{1}^{2}}, \gamma_{2}=\frac{p_{2} g_{2,2}}{p_{1} g_{1,2}+\sigma_{2}^{2}}$ & $\begin{array}{l}R_{1,1}=B \log _{2}\left(1+\gamma_{1}\right) \\
R_{2,2}=B \log _{2}\left(1+\gamma_{2}\right)\end{array}$ & $\begin{array}{l}\text { Interference } \\
\text { channel }\end{array}$ \\
\hline 2 & $\begin{array}{l}S B S_{2} \rightarrow U E_{1} \\
S B S_{1} \rightarrow U E_{2}\end{array}$ & $\gamma_{1}=\frac{p_{2} g_{2,1}}{p_{1} g_{1,1}+\sigma_{1}^{2}}, \gamma_{2}=\frac{p_{1} g_{1,2}}{p_{2} g_{2,2}+\sigma_{2}^{2}}$ & $\begin{array}{l}R_{2,1}=\operatorname{Blog}_{2}\left(1+\gamma_{1}\right) \\
R_{1,2}=\operatorname{Blog}_{2}\left(1+\gamma_{2}\right)\end{array}$ & $\begin{array}{l}\text { Interference } \\
\text { channel }\end{array}$ \\
\hline 3 & $\begin{array}{l}S B S_{1} \rightarrow U E_{1} \\
S B S_{2} \rightarrow U E_{1}\end{array}$ & $\begin{array}{l}\gamma_{1}=\frac{p_{1} g_{1,1}+2 \sqrt{p_{1} p_{2} g_{1,1} g_{2,1}}+p_{2} g_{2,1}}{\sigma_{1}^{2}} \\
\gamma_{2}=0\end{array}$ & $\begin{array}{l}R_{12,1}=B \log _{2}\left(1+\gamma_{1}\right) \\
R_{, 2}=0\end{array}$ & $\begin{array}{l}\text { Beamforming/ } \\
\text { diversity }\end{array}$ \\
\hline 4 & $\begin{array}{l}S B S_{1} \rightarrow U E_{2} \\
S B S_{2} \rightarrow U E_{2}\end{array}$ & $\begin{array}{l}\gamma_{1}=0, \\
\gamma_{2}=\frac{p_{1} g_{1,2}+2 \sqrt{p_{1} p_{2} g_{1,2} g_{2,2}}+p_{2} g_{2,2}}{\sigma_{2}^{2}}\end{array}$ & $\begin{array}{l}R_{, 1}=0 \\
R_{12,2}=B \log _{2}\left(1+\gamma_{2}\right)\end{array}$ & $\begin{array}{l}\text { Beamforming/ } \\
\text { diversity }\end{array}$ \\
\hline
\end{tabular}

by both SBSs, which are both relatively equidistant from each UE.

Since both SBSs have the same knowledge of all the channels involved, there are four different possibilities for serving both users. First, $U E_{1}$ and $U E_{2}$ may be served by $S B S_{1}$ and $S B S_{2}$ respectively. Second, $U E_{1}$ and $U E_{2}$ may be served by $S B S_{2}$ and $S B S_{1}$ respectively. Third, both SBSs may only serve $U E_{1}$. Fourth, both SBSs may only serve $U E_{2}$. While the first two cases are interference channels, the last two cases are beamforming channels under the power constraint of each SBS. The signal to interference plus noise ratio (SINR) of these cases is summarized in Table (7.1), where $\gamma_{i}$ is the SINR at $U E_{i}, g_{j, i}$ is the channel gain between $S B S_{j}$ and $U E_{i}$ and $p_{j}$ the transmit power from $S B S_{j}$. Moreover, $\sigma_{i}^{2}$ is the noise power plus interference from all other farther SBSs received by $U E_{i} . R_{j, i}$ is the achievable data rate from $S B S_{j}$ to $U E_{i}$. The SINR of the first two cases is trivial, however, the SINR of the last two cases can be broken down as follows. When both SBSs send the same data 
signal $s(t)$ to $U E_{i}$, the signal received would be:

$$
y(t)=h_{1, i} x_{1, i}+h_{2, i} x_{2, i}+n(t)
$$

where $h_{j, i}=\left|h_{j, i}\right| e^{\mathrm{j} \theta_{j, i}}$ is the channel impulse response from $S B S_{j}$ to $U E_{i} . x_{j, i}=$ $e^{-\mathrm{j} \theta_{j, i}} \sqrt{p_{j}} s(t)$ is the signal that radiated out of the antenna of $S B S_{j}$, and $n(t)$ includes noise process and interference signals from farther SBSs. Therefore,

$$
y(t)=\left(\left|h_{1, i}\right| \sqrt{p_{1}}+\left|h_{2, i}\right| \sqrt{p_{2}}\right) s(t)+n(t) .
$$

The power received from the desired signal would be

$$
\begin{aligned}
p_{r} & =\left(\left|h_{1, i}\right| \sqrt{p_{1}}+\left|h_{2, i}\right| \sqrt{p_{2}}\right)^{2}|s(t)|^{2} \\
& =\left|h_{1, i}\right|^{2} p_{1}+2\left|h_{1, i}\right|\left|h_{2, i}\right| \sqrt{p_{1} p_{2}}+\left|h_{2, i}\right|^{2} p_{2} .
\end{aligned}
$$

where $|s(t)|^{2}=1$. Let the channel gain $\left|h_{j, i}\right|^{2}=g_{j, i}$, the above equation confirms the SINR in the last two rows of Table (7.1).

Furthermore, the traffic of both users is assumed to arrive to both SBSs according to the Poisson process, with average packet arrival rates of $\lambda_{1}$ and $\lambda_{2}$ corresponding to $U E_{1}$ and $U E_{2}$, respectively. Both SBSs are assumed to apply a capacity achieving powerful coding scheme (e.g. polar or turbo code) such that each data packet is received correctly. In addition, it is assumed that all involved channels are independent processes following a Rayleigh distribution. Finally, the sub-band used by the macro base station is assumed to be orthogonal to the sub-band used in small cells (e.g. $[28,29])$. 


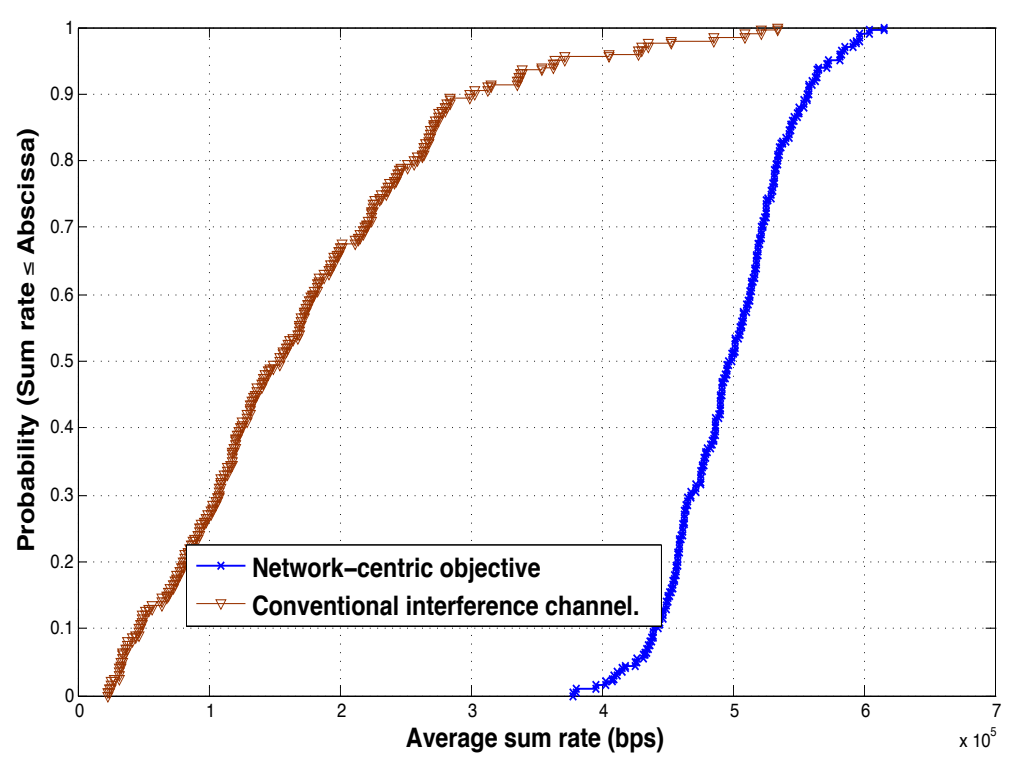

Figure 7.2: Cumulative density function (CDF) of the sum rate (simulation parameters are show in Table 7.2).

\subsection{Fully Distributed Scheduling Schemes}

In this section, we introduce different schemes of easy to solve optimization problems that harvest the diversity resulting from multiusers, in conjunction with the various ways of scheduling. We first start by focusing on the simple model shown in Fig. 7.1. In the following section, we extend this simple formulation to a more generalized and realistic formulation.

In contrast to conventional scheduling, the network-centric objective, which maximizes the sum rate without paying any attention to the fairness, is first introduced. We then introduce two cross layer-based schemes: a fair best effort scheduling scheme and a quality of service $\left(\mathrm{QoS}^{2}\right)$ guaranteed scheduling scheme.

\footnotetext{
${ }^{2}$ Satisfying packet delay requirements.
} 


\subsubsection{Network-Centric Objective (NCO)}

If the objective is to maximize the number of bits delivered without considering the performance (average packet delay) of individual users, then both SBSs would adapt the scheduling exclusively to the variations of the channels involved. This can be achieved by solving the following linear integer optimization problem.

$$
\begin{aligned}
& \underset{\mathbf{X}}{\operatorname{Maximize}}\left[\left(R_{1,1}+R_{2,2}\right) X_{1}+\left(R_{2,1}+R_{1,2}\right) X_{2}+R_{12,1} X_{3}+R_{12,2} X_{4}\right] \\
& \text { subject to } \\
& \sum_{k=1}^{4} X_{k}=1 \\
& \quad X_{k} \in\{0,1\}, \forall k=1, \ldots, 4 \text { (Variable space). }
\end{aligned}
$$

The objective function in (7.5) is to choose the type of scheduling that maximizes the sum rate. The first constraint is to make sure that only one scheduling type is chosen at any given time. This problem is optimized over the binary variable $\mathbf{X}$, as described by the second constraint. The optimization problem in (7.5) is a binary linear programming, and can therefore be solved using the Balas algorithm [60]. Since both SBSs have all the required channel information, both can solve the above problem and obtain the same solution. Thus, they can act coherently without any explicit coordination, as long as they have some sort of clock synchronization.

Figure (7.2) shows how the sum rate of this scheduling policy outperforms that of the conventional type of scheduling, where each UE is always attached to only one SBS (usually the closest). This improved performance is due to the fact that there are two SBSs adapting coherently to four channels at the same time. Therefore, as the number of SBSs and/or the number of UEs increases, the performance will improve even further. This scheme is perfect if both users can tolerate a large packet delay 
(e.g. email)

\subsubsection{Cross Layer-Based Scheduling Schemes}

\section{Fair Best Effort Scheme (FBE)}

Since the NCO policy favours the scheduling type that maximizes the sum rate and does not pay attention to individual performance, it is very possible that the user with bad geometry might end up with an accumulated queue and not be properly served. Therefore, we propose the following alternative, which takes into account the queue size of every user involved. Thus, if the queue of one user grows larger than the other, the FBE scheme will increase the opportunity of this user by involving a weighting factor to the achievable rate.

$$
\begin{aligned}
& \underset{\mathbf{X}}{\operatorname{Maximize}}\left[\left(L_{1} R_{1,1}+L_{2} R_{2,2}\right) X_{1}+\left(L_{1} R_{2,1}+L_{2} R_{1,2}\right) X_{2}+L_{1} R_{12,1} X_{3}+L_{2} R_{12,2} X_{4}\right] \\
& \text { subject to } \\
& \quad \sum_{k=1}^{4} X_{k}=1 \\
& \quad X_{k} \in\{0,1\}, \forall k=1, \ldots, 4 \text { (Variable space), }
\end{aligned}
$$

where $L_{i}$ is the queue length of $U E_{i}$ at the time of the scheduling decision making. The objective function in (7.6) is a weighted version of the objective function in the NCO scheme. The first constraint is to make sure that only one scheduling type is chosen at any given time. Again, this problem is optimized over the binary variable $\mathbf{X}$, as described by the second constraint.

NCO serves the sum rate maximizer user until either its geometry becomes less favourable or its queue becomes empty. On the other hand, FBE may leave the sum rate maximizer if the queue of the other user becomes relatively longer. Thus, NCO 
and FBE differ in the instances (timing) at which they transfer from one user to another.

\section{Satisfying a Quality of Service Requirement (SAT)}

In case one or more users have certain packet delay requirements, the scheduler has to adapt to channel variations and queue lengths at the same time. It needs to maximize the sum rate and satisfy users' requirements simultaneously. This can be achieved via the following optimization problem.

$$
\begin{aligned}
& \underset{\mathbf{X}}{\operatorname{Maximize}}\left[\left(R_{1,1}+R_{2,2}\right) X_{1}+\left(R_{2,1}+R_{1,2}\right) X_{2}+R_{12,1} X_{3}+R_{12,2} X_{4}\right] \\
& \text { subject to } \\
& \sum_{k=1}^{4} X_{k}=1 \\
& L_{1}+\lambda_{1} \tau-\frac{\tau}{M}\left(R_{1,1} X_{1}+R_{2,1} X_{2}+R_{12,1} X_{3}\right) \leq L_{1}^{\max } \\
& L_{2}+\lambda_{2} \tau-\frac{\tau}{M}\left(R_{2,2} X_{1}+R_{1,2} X_{2}+R_{12,2} X_{4}\right) \leq L_{2}^{\max } \\
& X_{k} \in\{0,1\}, \forall k=1, \ldots, 4
\end{aligned}
$$

where $\tau$ and $M$ are the duration of one time slot and the number of bits in one packet, respectively, and $L_{i}^{\max }$ is the maximum queue length that can be tolerated by $U E_{i}$. According to Little's law, $L_{i}^{\max }=\lambda_{i} D_{i}^{\max }$, where $D_{i}^{\max }$ is the maximum tolerated packet delay. Constraints $(7.7 \mathrm{~b}, \mathrm{c})$ are to make sure that the queue length of both users does not exceed the allowable limit at the end of each time slot. The left hand side of both constraints is the current queue length plus the expected number of arrival packets during that slot, minus the expected number of served (departure) packets. Constraints $(7.7$ a,d) are similar to Constraints (7.5 a,b). In contrary to NCO and FBE, the feasibility area of this problem (SAT) is sometimes empty, depending on 
the users' geometry and how sharp their requirements are.

\subsection{Generalized Formulation}

In this section, we generalize the above scheduling formulations to a more realistic system that consists of:

- A set of SBSs: $\mathcal{J}=\{1, \ldots, J\}$.

- A set of UEs: $\mathcal{N}=\{1, \ldots, N\}$.

- A set of subchannels: $\mathcal{C}=\{1, \ldots, C\}$.

Therefore, the maximum number of users that can be scheduled at any given time $t$ and over a given subchannel $c$ is $J$ UEs. Let $\mathcal{I}=\left\{\left(n_{1}, \ldots, n_{J}\right)_{i}\right\}_{i=1}^{i=I}$ be the set of all possible J-tuple combinations that can be made from $N$ different users. The size of this set is $I=\left(\begin{array}{c}N \\ J\end{array}\right)$, assuming $J \leq N$. For any given tuple of users, there will be $S$ different possibilities of scheduling, depending on the number of SBSs $J$. For example, if $J=2$, then $S=4$, as shown in Table 7.1. If $J=3$, then $S=27$, and so on. Therefore, $\mathcal{S}=\{1, \ldots, s, \ldots, S\}$ is the set of all the possible ways of scheduling. We can now introduce the optimization problem that governs the scheduling problem of $J$ SBSs that serve $N$ users over $C$ subchannels ${ }^{3}$.

\footnotetext{
${ }^{3}$ For brevity, here we generalize the formulation that corresponds to SAT in the previous section. Generalizations of NCO and FBE can be easily deduced.
} 
$\underset{\mathbf{X}}{\operatorname{Maximize}} \sum_{c=1}^{C} \sum_{i=1}^{I} \sum_{s=1}^{S}\left[\sum_{n \in i} R_{i, s, c}^{n}\right] X_{i, s, c}$

subject to

$$
\begin{aligned}
& \sum_{i=1}^{I} \sum_{s=1}^{S} X_{i, s, c}=1 ; \forall c \in \mathcal{C} \\
& L_{n}+\lambda_{n} \tau-\frac{\tau}{M}\left(\sum_{c=1}^{C} \sum_{i: n \in i} \sum_{s=1}^{S} R_{i, s, c}^{n} X_{i, s, c}\right) \leq L_{1}^{\max } ; \forall n \in \mathcal{N} \\
& X_{i, s, c} \in\{0,1\}, \forall i, s, \& c
\end{aligned}
$$

where $R_{i, s, c}^{n}$ is the rate of user $n$ over subchannel $c$ when tuple $i$ and scheduling policy $s$ are chosen. If user $n$ is not in tuple $i$, then $R_{i, s, c}^{n}=0$. On the other hand, if user $n$ is part of tuple $i$, the value of $R_{i, s, c}^{n}$ is defined by the scheduling $s$, as described in Table 7.1 for the case of $\mathrm{J}=2$ and $\mathrm{N}=2 . X_{i, s, c}$ is a binary variable whose value is one if UEs in tuple $i$ are chosen to transmit over subchannel $c$ according to the scheduling policy $s$, and zero otherwise. Constraint (7.8a) ensures that only one tuple is chosen to utilize subchannel $c$ according to exactly one scheduling policy. For every UE $n$, Constraint (7.8b) ensures its QoS satisfaction, in a similar fashion as in Equation (7.7 b,c) described in the previous section.

Problem (7.8) is a binary linear optimization problem, which can quickly be solved using the Balas algorithm [60]. One of the most realistic situations in small cells is when a couple of cell edge users are surrounded by $3 \mathrm{SBSs}$, and are assigned few subchannels. This novel solution can maximize the utilization of these few resources fairly and effectively. This solution is also applicable when $N \leq J$; this can be done by adding $J-N$ dummy users whose desired channel gain is zero. 
Table 7.2: Simulation parameters

\begin{tabular}{|c|c|}
\hline Parameters & Value \\
\hline \hline System frequency & $2 \mathrm{GHz}$ \\
\hline channel BW & $30 \mathrm{KHz}$ \\
\hline$p_{1}=p_{2}=p_{\max }$ & $1 \mathrm{mWatt}$ \\
\hline Small cell radius & $30 \mathrm{~m}$ \\
\hline Number of packets & 200 packets/user \\
\hline Packet size $(\mathrm{M})$ & $100 \mathrm{bits}$ \\
\hline slot duration $(\tau)$ & 1 millisecond \\
\hline Noise power spectral density & $-174 \mathrm{dBm} / \mathrm{Hz}$ \\
\hline Shadow fading deviation & $8 \mathrm{~dB}$ \\
\hline Small scale fading & Rayleigh fading \\
\hline Number of replication & 200 \\
\hline
\end{tabular}

\subsection{Simulation Results and Discussions}

The goal of this section is to evaluate the performance of the proposed algorithms. We simulated the exact system described in Section 7.1. The UEs are randomly distributed on the cells boundaries, which constitute $\% 10$ of the cell area. The simulation parameters are shown in Table 7.2. All schemes proposed in Section 7.2 are simulated, in addition to the conventional interference channel (IFC) (i.e., each user is attached to its closest SBS). Each scheme is set to deliver 200 packets to each user. Packets of $U E_{1}$ and $U E_{2}$ arrive to the SBSs according to Poisson processes with averages of $\lambda_{1}=1000$ packets/sec and $\lambda_{2}=1500$ packets/sec, respectively.

Each replication of our simulation starts by randomly deploying two users to the cells boundary, as shown in Fig. 7.1. Packets of both users are then generated, as well the channels realizations of $T$ time slots. $T$ is typically larger than the arrival time of the last packet. All of these generated processes are then used to test each proposed scheme. 
Table 7.3: Occurrence percentage of each type of scheduling as chosen by each proposed scheme.

\begin{tabular}{|c|c|c|c|c|}
\hline \multirow{2}{*}{ Scheme } & \multicolumn{4}{|c|}{ Percentage of occurrence (\%) } \\
\cline { 2 - 5 } & $X_{1}$ & $X_{2}$ & $X_{3}$ & $X_{4}$ \\
\hline \hline NOC & $610^{-2}$ & $310^{-2}$ & 55.65 & 44.26 \\
\hline FBE & $510^{-2}$ & $110^{-2}$ & 55.35 & 44.59 \\
\hline SATQoS & 9.09 & 5.51 & 31.06 & 54.34 \\
\hline
\end{tabular}

Figure 7.3.a shows the cumulative density function (CDFs) of the average time delay $^{4}$ of the packets for both users. Due to the very high range of values, the CDFs of the average time delay of packets delivered by the conventional interference channel is shown in Fig. 7.3.b, which clearly emphasizes the severe effects of inter-cell interference. Moreover, the CDFs of the sum rate of all schemes are shown in Fig. 7.4. Additionally, Table 7.3 shows how often each scheme selects each type of scheduling.

While the NCO performs best in terms of sum rates, its effect on the individual time delay, as shown in Fig. 7.3.a, is slightly inferior, with a high variance in the packets delays. On the other hand, the sum rate of FBE is slightly less than the NCO sum rate, but its impact on the individual time delay is much better than the NCOs impact. Nevertheless, Table 7.3 shows the similarities between NCO and FBE, in regards to how often they select each type of scheduling. In both schemes, the vast majority of scheduling types are $X_{3}$ or $X_{4}$, meaning that most of the time, only one user is served by both $\mathrm{SBSs}^{5}$.

The difference, which causes the time delay in FBE to be less than that in NCO, are the instances at which each scheme switches from serving one user to serving

\footnotetext{
${ }^{4}$ In this chapter, time delay includes the queuing delay in the SBS, and the transmission delay from the SBS to the user.

${ }^{5}$ This confirms the results in [41], which state that free-interference transmission maximizes the sum rate over multiple links.
} 
the other. The NCO serves the sum rate maximizing user until either its geometry becomes less favourable or its queue becomes empty, while the FBE may leave the sum rate maximizer if the queue of the other user becomes relatively longer. Thus, NCO and FBE differ in the instances (timing) at which they transfer from one user to another.

In all schemes except SAT, it is obvious that the time delay of the second user is larger than the time delay of the first user. This is because of the higher packet arrival rate $\left(\lambda_{2}=1500\right.$ pkts $\left./ \mathrm{sec}>\lambda_{1}=1000\right)$. In SAT, however, the second user whose packet arrival rate is higher enjoys the lowest time delay. This is due to the imposed constraints in the corresponding optimization problem (7.7). The extra constraints $(7.7 \mathrm{~b}, \mathrm{c})$ are the reason for the lower sum rate of SAT compared to that of NCO and FBE, as shown in Fig. 7.4. Moreover, in Table 7.3, the increase in $X_{1}$ and $X_{2}$ occurrences selected by SAT compared to NCO and FBE can be interpreted with the same reasoning.

The packet delays in SAT sometimes exceed the required limit (with a probability of about 0.1 ). This is because in our simulation, if the optimization problem (7.7) is infeasible, we resort to the best effort service using (7.6).

The CDFs of the total time required to deliver 400 packets to both cell edge users are shown in Fig. 7.5. NCO, FBE and SAT require an average total time of 200.9, 200.9 and 205 msec respectively, to deliver 200 packets to each user, with a variance of 195.2 , 195.2 and $496.8 \mathrm{msec}$ respectively. On the other hand, the conventional method of serving cell edge users requires on average $974.6 \mathrm{msec}$ with a variance of 1367.2 sec. Furthermore, we simulated the above schemes against various packet arrival rates. Figure 7.6 illustrates the average sum rate of different schemes with respect to the packet arrival rate. The noticeable increasing trend in the NCO sum rate is due to the fact that in the case of an empty queue, the NCO scheme will abandon the favoured user and serve the less favoured user whose queue is not empty. 
When the packet arrival rate is relatively large, the probability of leaving the favoured user due to an empty queue will decrease, and the sum rate will be higher than the case if the arrival rate is low.

In Fig. 7.6, we also notice that the sum rate of SAT approaches the sum rate of FBE, because if the optimization problem of SAT in (7.7) is infeasible, then we resort to the best effort service using the FBE optimization problem. When the packet arrival rate increases, satisfying constraints $(7.7$ b,c) becomes more difficult, and the probability of infeasibility increases, hence resorting to the FBE scheme more often. Moreover, the sum rate of the conventional IFC shows a decreasing pattern. This is because when the arrival rate is low, there is a greater chance that a user will have an empty queue and that the other user will experience a free interference transmission. When the packet arrival rate becomes large enough, the queues of both users will almost never be empty, and hence the IFC sum rate will be independent of the packet arrival rate. As a result, we can see in the same figure that the sum rate of the conventional IFC is constant for $\lambda>1500$ pkts/sec. Figure 7.7 shows the average packet delay with respect to the packet arrival rate. The packet time delay increases as the packets are quickly accumulated in the queue. The sharp increase in the delay resulting from the NCO scheme is due to the fact that after a certain arrival rate the queue of both users will be non-empty. Since NCO does not take the queue length into account, it will keep serving the geometrically favoured user, while the queue of the other user grows without limitations. Once the channel condition of the other user improves, $\mathrm{NCO}$ will switch to that user and repeat the same scenario. It is noticeable that the other scheme, which takes queue length into consideration, results in better packet delays. 


\subsection{Conclusions}

In this chapter, we exploited the proximity of the nodes in the small cells network to design fully distributed scheduling schemes that enhance the achievable rate of the cell edge users. The basic idea is to harvest a diversity gain resulting from having a number of users that can be serviced by various close SBSs, in many divers ways. We started the discussion by considering that two SBSs communicates data to two cell edge users over one subchannel, then we generalized it to include $J$ SBSs, $N$ users, and $C$ subchannels. Simulation results were presented to demonstrate the effectiveness of our proposed scheduling schemes.

As an extension of the proposed framework, multiple antennas architecture can even be combined with these schemes with the objective of harvesting the benefits of space diversity/beamforming/spatial multiplexing. 


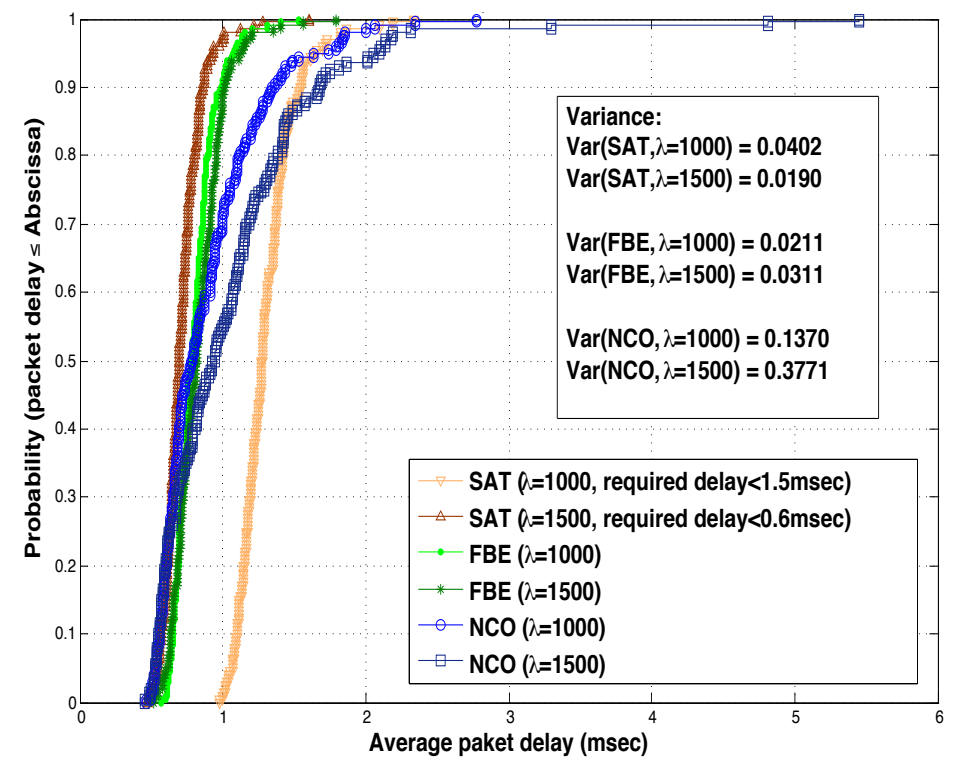

(a)

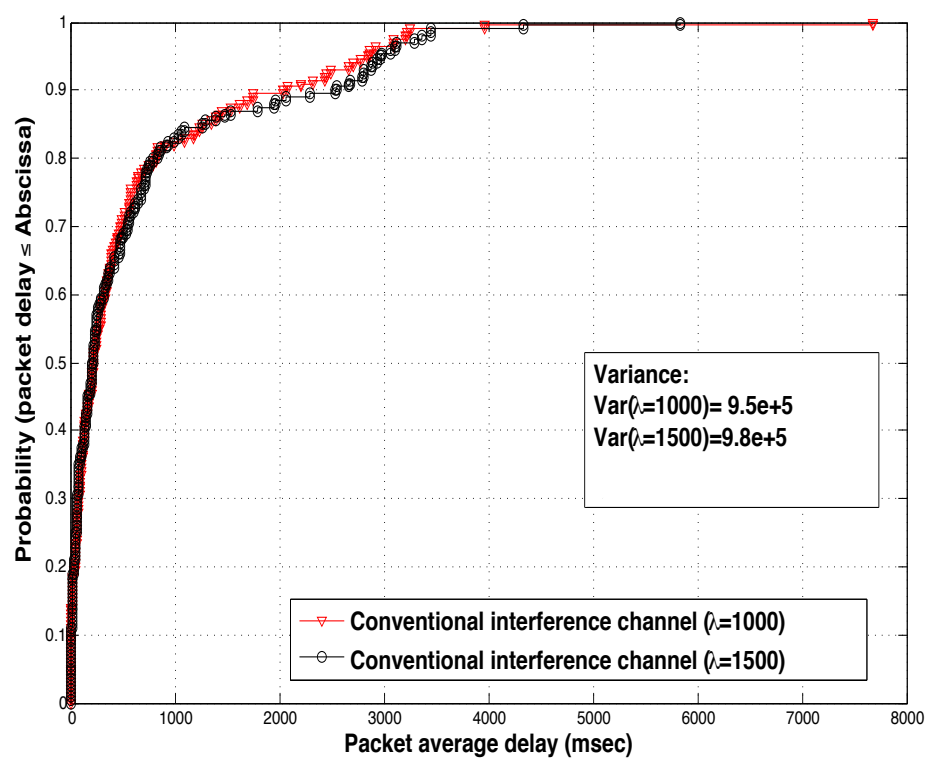

(b)

Figure 7.3: (a) CDFs of the packet time delay of different proposed scheduling schemes; (b) CDFs of the packet time delay when the conventional interference channel scheduling is used.

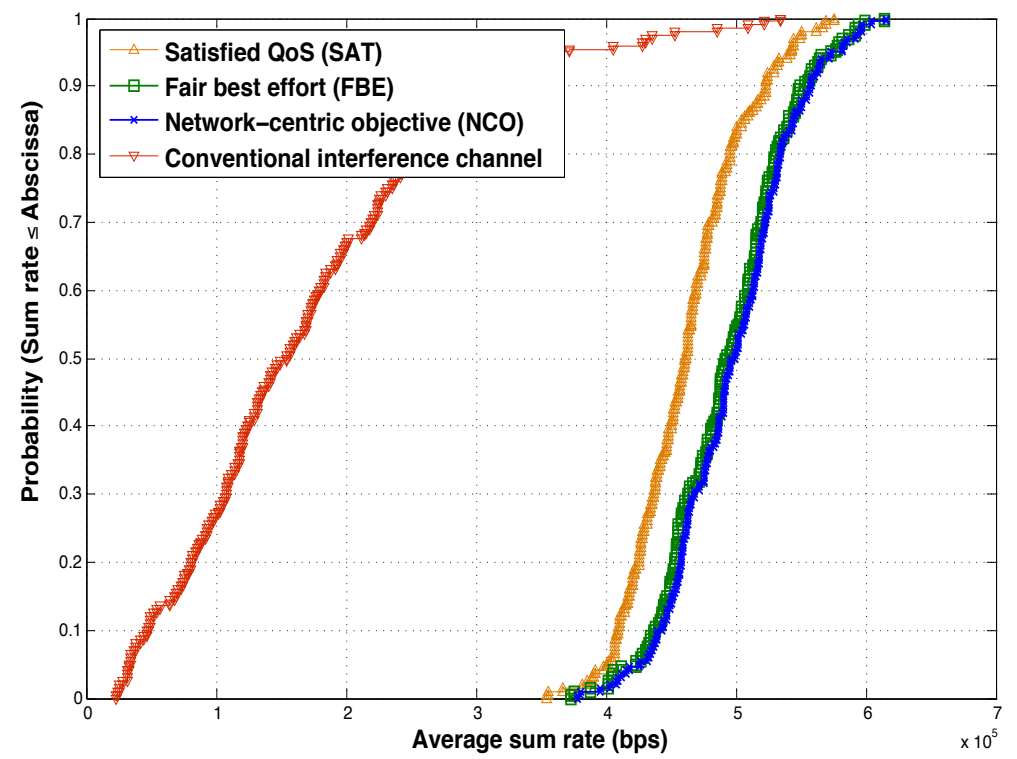

Figure 7.4: CDFs of cell edge users' sum rates for different schemes. 


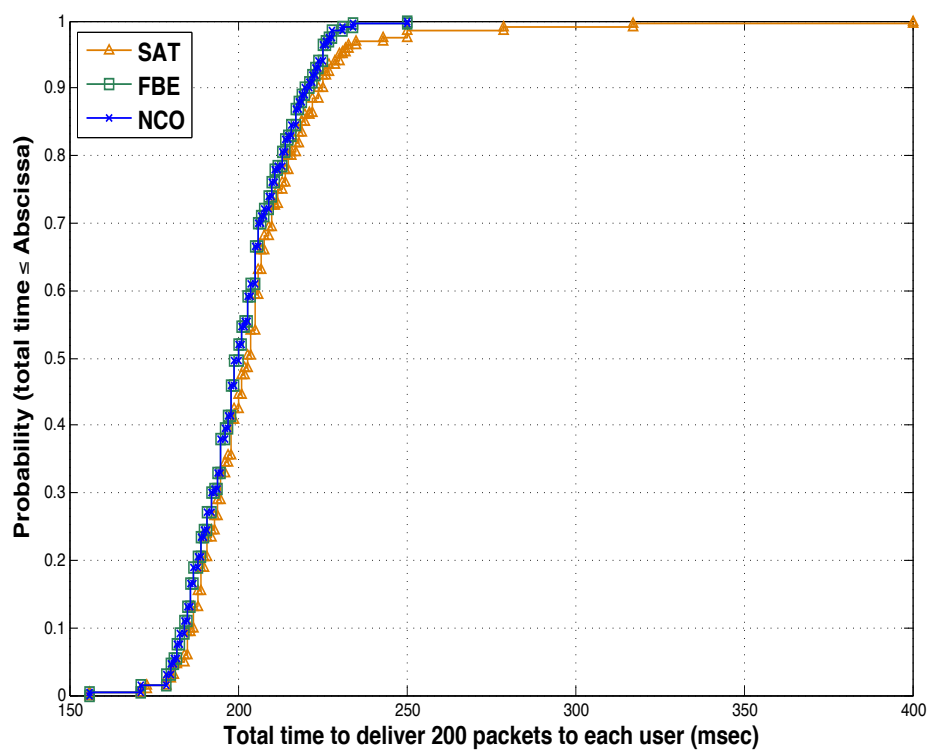

(a)

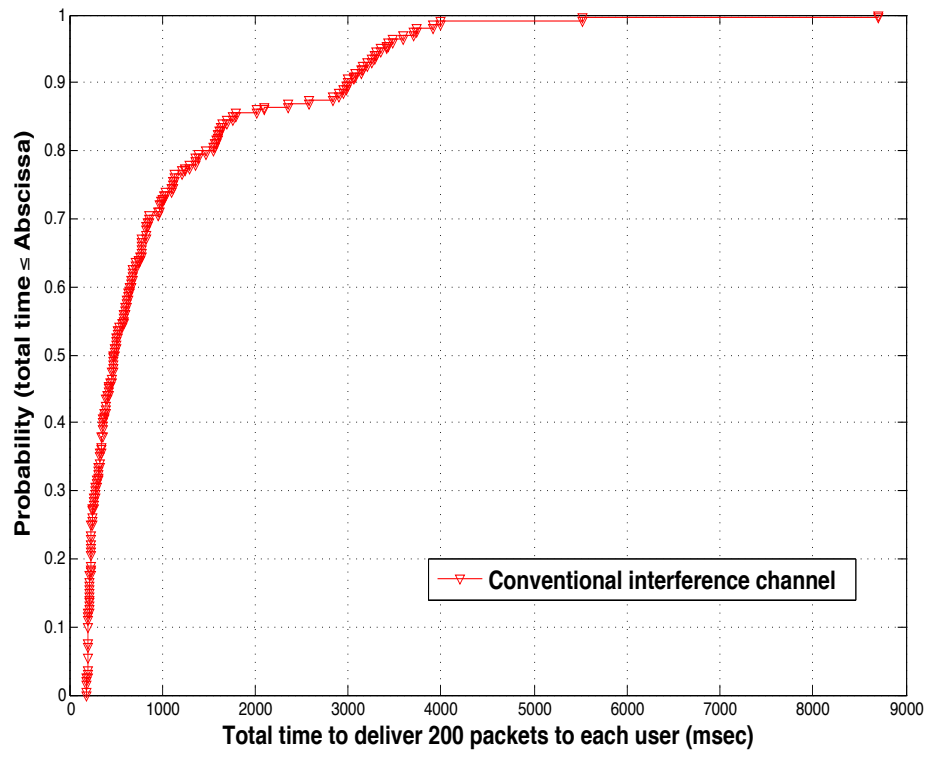

(b)

Figure 7.5: CDFs of the total time required to deliver 200 packets. (a) Different proposed scheduling schemes, (b) Conventional interference channel scheduling.

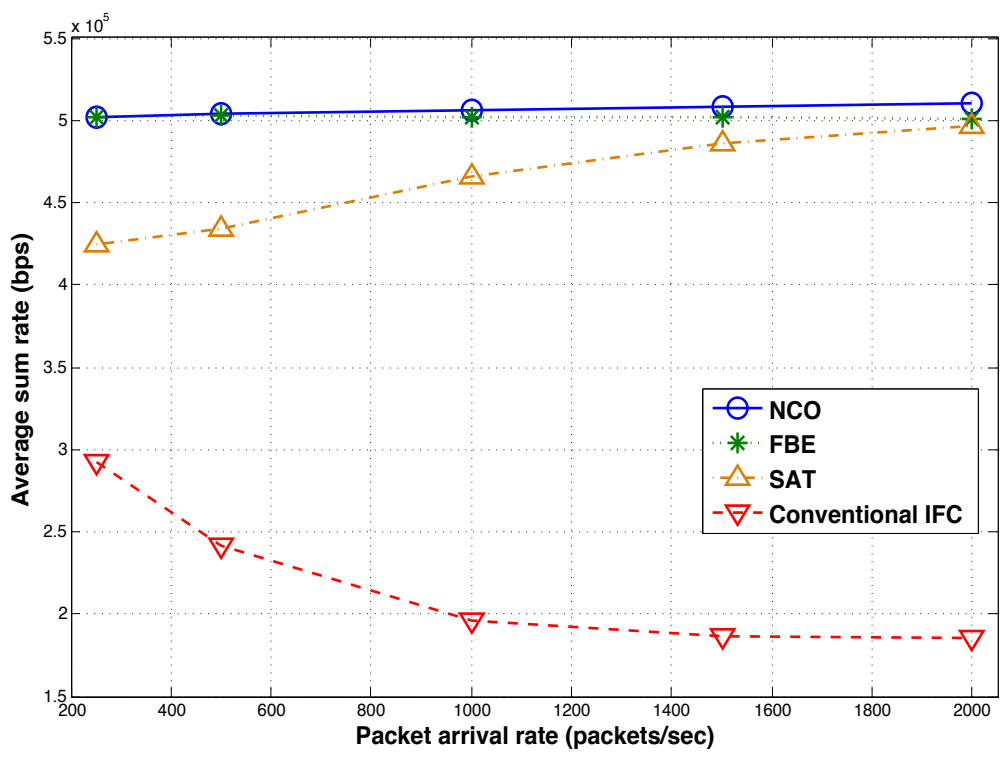

Figure 7.6: The sum rate vs. the packet arrival rate. 


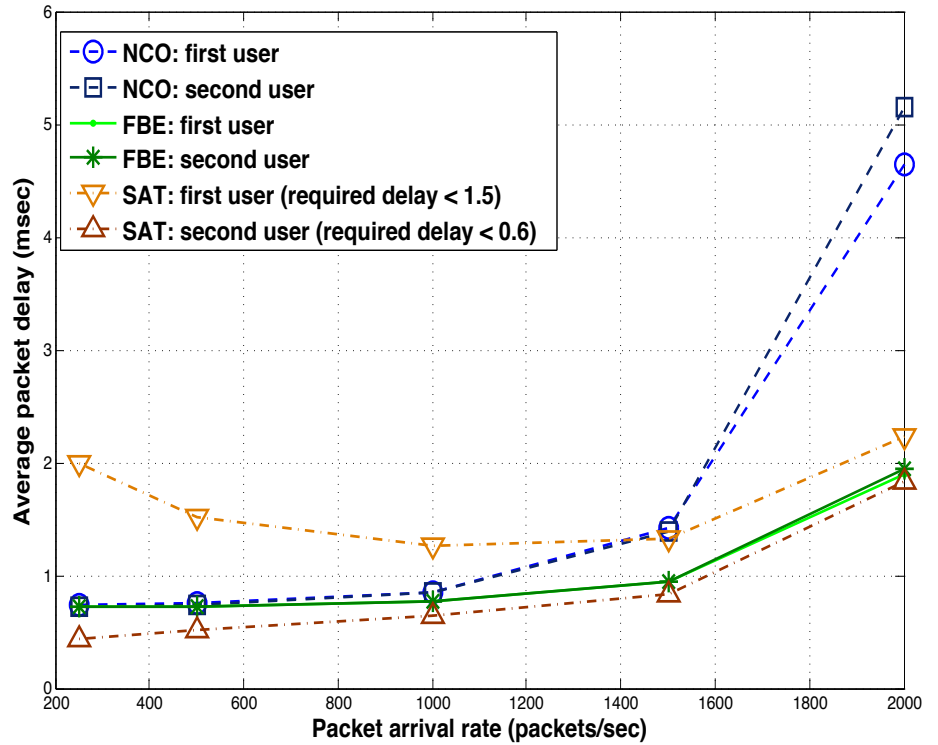

(a)

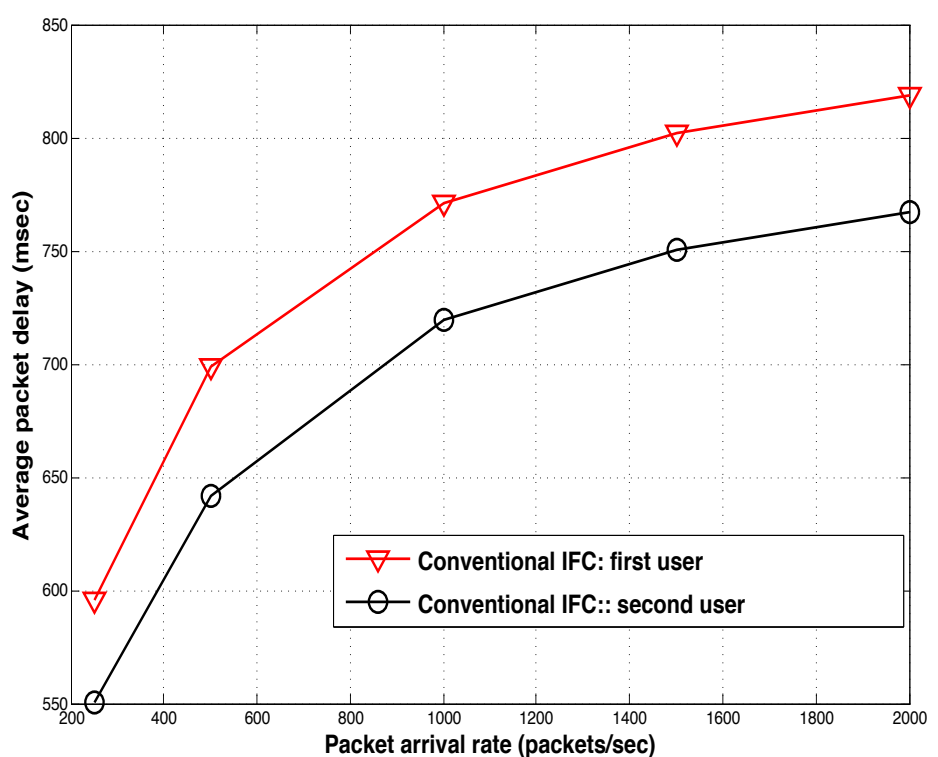

(b)

Figure 7.7: Average packet delay. (a) Different proposed scheduling schemes, (b) Conventional interference channel scheduling. 


\section{Chapter 8}

\section{Conclusion and Research Directions}

To meet the increasing demand for wireless services, spectrum reuse through dense small cells deployment is becoming increasingly inevitable because of its promising payoff compared to other solutions. However, one of the greatest challenges that comes along with the anticipated payoff is the co-channel interference. Optimal interference control in such a dense network using centralized and global approaches is very prohibitive due to scalability issues and massive overhead costs. This thesis introduced the problem of inter-cell interference in heterogeneous networks. After introducing a required background and literature review, four different distributed solutions for inter-cell interference management in HetNet systems were proposed.

In Chapter 4, we proposed a novel energy-efficient resource allocation and interference control scheme for green heterogeneous networks using the game theoretic approach. Since macrocell users may have higher priority from an operators viewpoint, their QoS requirements need to be met. However, the MBS operation is not as efficient as that of small cells in terms of spectrum and energy efficiency. Therefore, an approximate optimal resource allocation at the macrocell level, which assigns the minimal required resources to the macrocell users, was proposed. The remaining bulk of the subchannels is then shared by all small cells using a game-based scheme. Using economic concepts, we designed the utility function of every user to include 
two parts, a reward part and a cost part, the latter being quadratic with respect to the transmit power. The action of every co-channel small cell user is to transmit at an optimal power level, seeking to maximize its own utility. Such a strategy leads to justified interference in the air. The game analysis demonstrated that the proposed non-cooperative game has a unique Nash equilibrium point.

Furthermore, for a dense small cells network, Chapter 5 introduced a scheme by which the MBS is assigned a minimum number of radio resources to satisfy the required QoS. Then, the remaining subchannels are assigned to all SBSs. To control the co-layer interference, a mean-field game-based scheme, in which the total power of each player is optimally distributed over the subchannels, was introduced. A new dimension of frequency diversity is created in the interference term by exploiting the statistics of the interfering channels as well as applying a mean-field approximation. Higher spectral efficiency is attained by taking advantage of both frequency diversities in the desired channel, as well as in the interference channel.

Considering a dense network of a large number of small cells, Chapter 6 proposed an interference control scheme with two-levels of algorithms. The first is an overlay algorithm in which the MBS is modelled as the leader and the set of all SBSs is modelled as the follower. The second algorithm is based on a non-cooperative game in which the players are the SBSs. While the goal of the former algorithm is to control the cross-layer interference, the latter is to alleviate the co-layer interference. Using recent advances in mean-field game theory, we decoupled the complex system into localized optimization problems. Each base station selects its policy by using only its local information and some macroscopic information.

The proximity of the nodes in the small cells networks is exploited in Chapter 7 to design fully distributed scheduling schemes that improve the achievable rate of the cell edge users. The basic idea is to harvest a diversity gain resulted from having a number of users that can be serviced by various close SBSs, in many divers ways. The 
discussion started by considering that two SBSs communicate data to two cell edge users over one subchannel; it was then generalized to include the arbitrary number of SBSs, users, and subchannels. Simulation results were presented to demonstrate the effectiveness of the proposed scheduling schemes.

Adaptivity and autonomy are two major features of self organized networks $(\mathrm{SON})$. It is to be noted that all of the schemes introduced in this thesis possess both of these features. In Section 8.1, we discuss the possible extensions of the work presented throughout this thesis. Furthermore, in the future, we will focus on contemporary trends for the wireless networking research. Thus, Section 8.2 includes some relevant research directions.

\subsection{Extensions to the Proposed Research}

\subsubsection{Priority-Less Schemes}

In the proposed research, macrocell nodes have been given higher priority than small cells for logical reasons. In fact, several other studies have created some kind of advantage for MBS over SBSs [2,26,28,29]. Considering a more general case, a priority-less interference management scheme in which all nodes have the same priority for access to the system resources, may be a potential extension.

\subsubsection{Incorporating a Medium Access Control (MAC) Pro- tocol}

In the proposed interference management solutions, a MAC protocol in the MBS is fully described, however, it has been left arbitrary at the small cells level. That is due to the fact that our proposed schemes are intended to address the fundamental interference management problem thoroughly seeking, in the first place, an efficient 
co-existence between MBS and several SBSs. Indeed, leaving the MAC protocol arbitrary, while dealing with the interference problem, is common in the literature, see for example [2] and [41]. Nevertheless, scheduling is an important factor in order to enhance spectral efficiencies. Therefore, one important extension to the proposed work is incorporating some MAC protocol into interference management at the small cells level. Indeed, integrating a suitable MAC protocol with the proposed interference control mechanisms, and having them coherently interact, would be greatly beneficial.

\subsubsection{Stochastic Dynamic Games}

In this thesis, games have been designed as one shot games. One possible extension to the game in Chapter 4 is converting it into a dynamic stochastic game. In the dynamic game, a plan for several time slots is made in advance. This kind of game is called a repeated game, where random variations in the time domain are exploited. Stochastic dynamic games can be solved by computing the Markov perfect equilibrium (MPE), which is typically obtained via a dynamic programming algorithm. However, when there are more than a few players taking part in the game, the curse of dimensionality makes dynamic programming algorithms intractable. Thus, a method for approximating MPE is important to reduce the level of complexity. This leads us to the possible extension for the game introduced in Chapter 6 . The dynamic mean-field game, in which the MFG is played repeatedly, provides a good approximation for MPE $[11-14,16]$.

\subsubsection{Device-to-Device Communication}

Device-to-Device (D2D) communication is an underlay technology to the cellular network to increase the spectral efficiency. In D2D communication, UEs transmit data signals to each other over a direct link using the cellular resources instead of 
through the BS, but are controlled under the BS. Accordingly, in a given area, a large number of D2D active links is expected. This is very similar to the system modelled in Chapters 5 and 6 if each SBS is considered a normal device (i.e., without MAC protocol and connected only with one UE). Therefore, the potential of improving spectral utilization of D2D via mean-field game theory deserves more attention. The only requirement is that the $\mathrm{BS}$ needs to feed the devices with the number of active links at every time slot.

\subsection{Relevant Research Directions}

\subsubsection{Densification Limits}

The fundamental limits of densifying the network with SBSs are not fully understood. If the density of SBSs increases, can the frequency reuse gain increase indefinitely? Or, would it saturate at some point due to the interference? Further research on the fundamental limits of small BSs densification would be extremely valuable.

\subsubsection{Exploiting Traffic Patterns in Partitioning Based Schemes}

Small base stations usually serve fewer users than macro base stations, and hence lack traffic aggregation. Therefore, in a few msec., their queues of packets would alternate between empty and non-empty states. In schemes where resources are partitioned, the resources allocated to an SBS whose queue is empty in the current time slot should be used by adjacent SBSs. Therefore, a possible research direction is to design a viable method of accessing the spectrums of idle SBSs. 


\subsubsection{Learning the Behaviours of Neighboring SBSs}

In [29], the authors proposed orthogonal resource partitioning between MBS and SBSs. They further suggested a random access technique in frequency domain among SBSs, they called it F-ALLOHA. In F-ALLOH, SBSs randomly access the allotted spectrum with the hope that adjacent small cells will not consistently access the same subchannel at the same time. We suggest that each SBS learns the behaviours of its adjacent SBSs, whose owners usually have repeated habits, and collects the statistics of their traffic pattern. Designing access algorithms based on the collected traffic information would make the access more deliberate instead of purely random, and hence reduce the probability of collision.

Furthermore, exploiting the traffic pattern of small cells may also help MBS. For example, during parts of the day (e.g. morning time), MBS in a residential area may access parts of the spectrum allotted to SBSs since most of users are outside. On the other hand, MBS located in a business area may access most of SBSs spectrum at night as very few employees will remain in their offices.

\subsubsection{MIMO Small Cells}

SBSs can perform temporal link adaptation using adaptive modulation and coding schemes. In addition, SBSs can further perform spatial link adaptation using multiple input multiple output (MIMO) antennas. In the latter technique, an SBS may switch between providing high data rates and reliable transmission. A high data rate is accomplished by spatial multiplexing in high SINR channels, while robust and reliable transmission can be achieved by diversity and beamforming techniques in low SINR channels. Therefore, designing link adaptive MIMO switching methods in SBSs would be interesting research. 


\subsubsection{Joint Beamforming and Scheduling}

Beamforming $(\mathrm{BF})$ techniques are known to reduce the co-channel interference. The basic idea of a beam former is focusing the energy towards a desired user and simultaneously nulling this signal in the direction of the other users. It is very possible that the gain of BF could be significantly increased if the scheduling is taken into account. With an interference prediction mechanism, each BS can attain higher gains if it jointly schedules the users and computes the beamforming vectors. 


\section{List of References}

[1] F. Edalat, J. K. Tan, K. M. Nguyen, N. Matalon, and C. G. Sodini, "Measured data rate from adaptive modulation in wideband ofdm systems," in Proc. The 2006 IEEE 2006 International Conference on Ultra-Wideband, Waltham, MA, pp. 195-200, Sept. 2006.

[2] V. Chandrasekhar, J. Andrews, T. Muharemovic, Z. Shen, and A. Gatherer, "Power control in two-tier femtocell networks," IEEE Trans. Wireless Comm., vol. 8, pp. 4316 - 4328, Aug. 2009.

[3] D. Lopez-Perez, A. Valcarce, G. de la Roche, and J. Zhang, "OFDMA femtocells: A roadmap on interference avoidance," IEEE Comm. Magazine, vol. 47, pp. 4148, Apr. 2009.

[4] P. Xia, V. Chandrasekhar, and J. Andrews, "Open vs. closed access femtocells in the uplink," IEEE Trans. Wireless Comm., vol. 9, pp. 3798 - 3809, Dec. 2010.

[5] International Telecommunication Union (ITU), "Guidelines for evaluation of radio transmission technologies for imt-2000," ITU Recommendation M.1225, www.itu.int/ITU-R.

[6] A. Goldsmith, Wireless Communication. Cambridge University Press, First edition, 2005.

[7] T. S. Rappaport, Wireless Communication: principles and practice. Prentice Hall PTR, Second edition, 2002.

[8] A. Goldsmith and S. Chua, "Variable-rate variable-power mqam for fading channels," IEEE Transations on Communications., vol. 45, pp. 1218-1230, Oct. 1997.

[9] S. Chung and A. Goldsmith, "Degrees of freedom in adaptive modulation: A unified view," IEEE Transations on Communications., vol. 49, pp. 1561-1571, Sep. 2001. 
[10] M. J. Osborne, An Introduction to Game Theory. Oxford University Press, 2000.

[11] S. Adlakha and R. Johari, "Mean field equilibrium in dynamic games with complementarities," in Proc. 49th IEEE Conference on Decision and Control (CDC), 2010.

[12] V. Abhishek, S. Adlakha, R. Johari, and G. Weintraub, "Oblivious equilibrium for general stochastic games with many players," in Proc. Allerton Conference on Communication, Control and Computing, 2007.

[13] M. Huang, "Mean field stochastic games with discrete states and mixed players," in Proc. GameNets, Vancouver, May 2011.

[14] O. Gueant, J. M. Lasry, and P. L. Lions, "Mean field games and applications," in Lecture Notes in Math, Springer, Berlin, pp. 205-266, 2011.

[15] R. Wilde and S. Singh, Statistical Mechanics: Fundamentals and Modern Applications. John Wiley \& Sons, 1998.

[16] G. Y.Weintraub, L. Benkard, and B. V. Roy, "Oblivious equilibrium: A mean field approximation for large-scale dynamic games," Advances in Neural Information Processing Systems, vol. 47, 2006.

[17] S. Adlakha, R. Johari, G. Weintraub, and A. Goldsmith, "A mean field approach to competition in large scale wireless systems," in ACM Mobihoc S3 Workshop, New Orleans, LA., 2009.

[18] N. J. Crawford, "Mean field theories and models of statistical physics," $P h D$ dissertation, University of California, Los Angeles, 2007.

[19] J. Andrews, "Interference cancellation for cellular systems: a contemporary overview," IEEE Wireless Comm., vol. 12, pp. 19-29, Apr. 2005.

[20] J. Andrews, W. Choi, and R. Heath, "Overcoming interference in spatial multiplexing MIMO cellular networks," IEEE Wireless Comm., vol. 14, pp. 95-104, 2007.

[21] G. Boudreau, J. Panicker, N. Guo, R. Chang, N. Wang, and S. Vrzic, "Interference coordination and cancellation for $4 \mathrm{G}$ networks," IEEE Comm. Magazine, vol. 47, pp. 74-81, Apr. 2009.

[22] M. Rahman and H. Yanikomeroglu, "Enhancing cell-edge performance: a downlink dynamic interference avoidance scheme with inter-cell coordination," IEEE Trans. Wireless Comm., vol. 9, pp. 1414-1425, Apr. 2010. 
[23] R. Madan, J. Borran, A. Sampath, N. Bhushan, A. Khandekar, and T. Ji, "Cell association and interference coordination in heterogeneous LTE-A cellular networks," IEEE J. Sel. Areas Commun., vol. 28, pp. 1479-1489, Dec. 2010.

[24] D. Lopez-Perez, A. Ladanyi, A. Juttner, H. Rivano, and J. Zhang, "Optimization method for the joint allocation of modulation schemes, coding rates, resource blocks and power in self-organizing LTE networks," in Proc. IEEE INFOCOM'11, pp. 111-115, Apr. 2011.

[25] K. song Lee and D.-H. Cho, "Cooperation based resource allocation for improving inter-cell fairness in femtocell systems," in Proc. IEEE PIMRC'10, pp. 11681172, Sep. 2010.

[26] S. Park, W. Seo, Y. Kim, S. Lim, and D. Hong, "Beam subset selection strategy for interference reduction in two-tier femtocell networks," IEEE Trans. Wireless Comm., vol. 9, pp. 3440 - 3449, Nov. 2010.

[27] J. Vicario, R. Bosisio, C. Anton-Haro, and U. Spagnolini, "Beam selection strategies for orthogonal random beamforming in sparse networks," IEEE Trans. Wireless Comm., vol. 7, pp. 3385-3396, Sep. 2008.

[28] Y. Kim, T. Kwon, and D. Hong, "Area spectral efficiency of shared spectrum hierarchical cell structure networks," IEEE Trans. Vehicular Technology., vol. 59, pp. 4145-4151, Oct. 2010.

[29] V. Chandrasekhar and J. Andrews, "Spectrum allocation in tiered cellular networks," IEEE Trans. Communications., vol. 57, pp. 3059 - 3068, Oct. 2009.

[30] H.-S. Jo, C. Mun, J. Moon, and J.-G. Yook, "Interference mitigation using uplink power control for two-tier femtocell networks," IEEE Trans. on Wireless Commun., vol. 8, pp. 4906-4910, Oct. 2009.

[31] R. Xie, F. R. Yu, H. Ji, and Y. Li, "Energy-efficient resource allocation for heterogeneous cognitive radio networks with femtocells," IEEE Trans. Wireless Commun., vol. 11, pp. 3910 -3920, Nov. 2012.

[32] X. Kang, R. Zhang, and M. Motani, "Price-based resource allocation for spectrum-sharing femtocell networks: A stackelberg game approach," IEEE J. Sel. Areas Comm., vol. 30, pp. 538-549, Apr. 2012.

[33] S. Haykin, "Cognitive radio: brain-empowered wireless communications," IEEE Journal on Selected Areas in Commun., vol. 23, pp. 201 - 220, Feb. 2005. 
[34] V. Jungnickel, K. Manolakis, W. Zirwas, B. Panzner, V. Braun, M. Lossow, M. Sternad, R. Apelfrjd, and T. Svensson, "The role of small cells, coordinated multipoint, and massive mimo in 5g," IEEE Comm. Mag., pp. 44- 51, May 2014.

[35] R. Yates, "A framework for uplink power control in cellular radio systems," IEEE J. Sel. Areas Commun., vol. 13, pp. 1341-1347, 1995.

[36] M. Rahman and H. Yanikomeroglu, "Interference avoidance through dynamic downlink ofdma subchannel allocation using intercell coordination," in Proc. IEEE Vehicular Technology conference (VTC), pp. 1630-1635, Spring 2008.

[37] A. Stolyar and H. Viswanathan, "Self-organizing dynamic fractional frequency reuse in ofdma systems," in Proc. IEEE INFOCOM, pp. 691-699, Apr. 2008.

[38] E. C. van der Meulen, "A survey of multi-way channels in information theory, 1961-1976," IEEE Trans. Info. Theory, vol. 23, pp. 1-37, Jan. 1977.

[39] F. Meshkati, H. V. Poor, and S. Schwartz, "Energy-efficient resource allocation in wireless networks," IEEE Signal Processing Magazine, vol. 24, pp. 58-68, May 2007.

[40] D. Goodman and N. Mandayam, "Power control for wireless data," in Proc. IEEE International Workshop on Mobile Multimedia Commun., pp. 55-63, Nov. 1999.

[41] A. Gjendemsj, D. Gesbert, G. Oien, and S. Kiani, "Binary power control for sum rate maximization over multiple interfering links," IEEE Trans. on Wireless Commun., vol. 7, pp. 3164-3173, Aug. 2008.

[42] S. Leyffer, Deterministic Methods for Mixed Integer Nonlinear Programming. PhD thesis, University of Dundee, Dundee, UK, 1993.

[43] H. W. Kuhn, "The hungarian method for the assignment problem," Naval Research Logistics Quarterly, vol. 2, pp. 83-97, 1955.

[44] D. Falomari, N. Mandayam, and D. Goodman, "A new framework for power control in wireless data networks: Games utility and pricing," in Proc. Allerton Conf. on Communication, Control and Computing, Illinois, USA, pp. 546-555, Sept. 1998.

[45] V. Shah, N. Mandayam, and D. Goodman, "Power control for wireless data based on utility and pricing," in Proc. IEEE PIMRC'98, pp. 1427-1432, Sept. 1998. 
[46] H. Ji and C. Huang, "Non-cooperative uplink power control in cellular radio systems," Wireless Networks, vol. 4, pp. 233-240, March 1998.

[47] A. W. Roberts and D. Varberg, Convex Functions. New York: Academic, 1973.

[48] J. Rosen, "Existence and uniqueness of equilibrium points for concave n-person games," Econometrica, vol. 33, pp. 520-534, 1965.

[49] D. Bertsekas and J. Tsitsiklis, Parallel and Distributed Computation: Numerical Methods. Athena Scientific, Massachusetts, 1997.

[50] C. Saraydar, N. Mandayam, and D. Goodman, "Efficient power control via pricing in wireless data networks," IEEE Trans. on Comm., vol. 50, pp. 291-303, Feb. 2002.

[51] D. W. K. Ng, E. S. Lo, and R. Schober, "Energy-efficient resource allocation in multi-cell OFDMA systems with limited backhaul capacity," IEEE Trans. Wireless Commun., vol. 11, pp. 3618-3631, Oct. 2012.

[52] J. G. Andrews, H. Claussen, M. Dohler, S. Rangan, and M. C. Reed, "Femtocells: Past, present, and future," IEEE J. Sel. Areas Comm., vol. 30, pp. 497-508, Apr. 2012.

[53] L. Scalia, K. K. T. Biermann, C. Choi, and W. Kellerer, "Power-efficient mobile backhaul design for CoMP support in future wireless access systems," in Proc. IEEE INFOCOM Workshop on Green Comm. Networking, (Shanghai, China), Apr. 2011.

[54] F. Diehm and G. Fettweis, "On the impact of signaling delays on the performance of centralized scheduling for joint detection cooperative cellular systems," in Proc. IEEE WCNC'11, (Cancun, Mexico), Mar. 2011.

[55] A. Goldsmith, M. Effros, R. Koetter, M. Medard, A. Ozdaglar, and L. Zheng, "Beyond Shannon: the quest for fundamental performance limits of wireless ad hoc networks," IEEE Comm. Mag., vol. 49, pp. 195 -205, May 2011.

[56] M. Huang, P. Caines, and R. Malhame, "The NCE (mean field) principle with locality dependent cost interactions," IEEE Trans. Auto. Control, vol. 55, pp. 2799-2805, Dec. 2010.

[57] M. Y. Huang, "Mean field stochastic games with discrete states and mixed players," in Proc. GameNets, (Vancouver, BC, Canada), May 2012. 
[58] F. Meriaux, V. Varma, and S. Lasaulce, "Mean field energy games in wireless networks," in Proc. Asilomar Conf. on Signals, Systems, and Computers, (Pacific Grove, CA), Nov. 2012.

[59] H. Tembine, P. Vilanova, M. Assaad, and M. Debbah, "Mean field stochastic games for SINR-based medium access control," in Proc. 5th Int'l ICST Conf. on Performance Evaluation Methodologies and Tools (VALUETOOLS'11), (Cachan, France), May 2011.

[60] E. Balas, "An additive algorithm for solving linear programs with zero-one variables," Operation Res., vol. 13, pp. 517-546, 1965.

[61] C. Wong, R. Cheng, K. B. Letaief, and R. Murch, "Multiuser ofdm with adaptive subcarrier, bit, and power allocation," IEEE Journal on Selected Areas in Commun., vol. 17, pp. 1747 - 1758, Oct. 1999.

[62] W. Rhee and J. Cioffi, "Increase in capacity of multiuser ofdm system using dynamic subchannel allocation," in Proc. IEEE Vehicular Technology Conf. (VTC2000-Spring), Tokyo, Japan, May 2000.

[63] D. Niyato and E. Hossain, "Adaptive fair subcarrier/rate allocation in multi rate ofdma networks: radio link level queuing performance analysis," IEEE Trans. Veh. Technol., vol. 55, pp. 1897-1907, Nov. 2006.

[64] K. Abhishek, S. Leyffer, and J. Linderoth, "Filmint: An outer approximationbased solver for convex mixed-integer nonlinear programs," INFORMS Journal on Computing, vol. 22, pp. 555-567, 2010.

[65] M. Hunting, "Solving convex minlp problems with aimms," An AIMMS white paper, Paragon Decision Technology, BV, Aug. 2012.

[66] M. Abramovitz and I. Stegun, Handbook of Mathematical Functions with Formulas, Graphs, and Mathematical Tables. Dover, New York, 1964.

[67] M. Schubert and H. Boche, "A generic approach to qos-based transceiver optimization," IEEE Trans. on Commun., vol. 55, pp. 1557-1566, Aug. 2007.

[68] S. Adlakha, R. Johari, G. Weintraub, and A. Goldsmith, "On oblivious equilibrium in large population stochastic games," in Proc. IEEE Conference on Decision and Control, pp. 3117-3124, Dec 2010. 
[69] F. Meriaux and S. Lasaulce, "Mean-field games and green power control," in Proc. 5th International Conference on Network Games, Control and Optimization (NetGCooP), pp. 1-5, Oct. 2011.

[70] T. Han and K. Kobayashi, "A new achievable rate region for the interference channel," IEEE Trans. Inf. Theory, vol. 27, pp. 49 -60, Jan. 1981.

[71] M. H. Costa, "On the gaussian interference channel," IEEE Trans. Inf. Theory, vol. 31, pp. $607-615$, Sep. 1984.

[72] M. Charafeddine, A. Sezgin, and A. Paulraj, "Rate region frontiers for n-user interference channel with interference and noise," in Proc. 45th Allerton Conf. Communication, Control and Computing, (Monticello, IL,), Sep. 2007.

[73] R. Irmer, H. Droste, P. Marsch, M. Grieger, G. Fettweis, S. Brueck, H.-P. Mayer, L. Thiele, and V. Jungnickel, "Coordinated multipoint: concepts, performance, and field trial results," IEEE Communications Magazine, vol. 49, pp. 102-111, Feb. 2011. 


\section{Appendix A}

\section{Simulation Setup}

In this appendix, we explain the general procedure that was followed for the thesis simulation; this procedure differs depending on the type of network deployment used.

\section{A.1 Network Deployment}

\section{Organized topology:}

The motivation of this topology is that sometimes, all SBSs are under the authority of one body. In this case, that body will carefully selects the locations of the SBSs. This reflects the case of picocells or the case of femto cells under the authority of one enterprise.

In a given area $\mathcal{A}$, small cells are organized based on the network density. Fig. A.1.a \& b show an organized topology for sparse networks and dense networks, respectively. The MBS is deployed in the centre of area $\mathcal{A}$, while the locations of its associated MUs are picked using a uniform random distribution. The SBSs are installed in the centre of the small cells, and each user in every cell is deployed randomly, within that cell, using a uniform random distribution. In Fig. A.1, the different colours of the adjacent small cells indicate the orthogonal frequency bands used in each cell, when Reuse-3 or SFR are employed to control the inter-cell interference. 


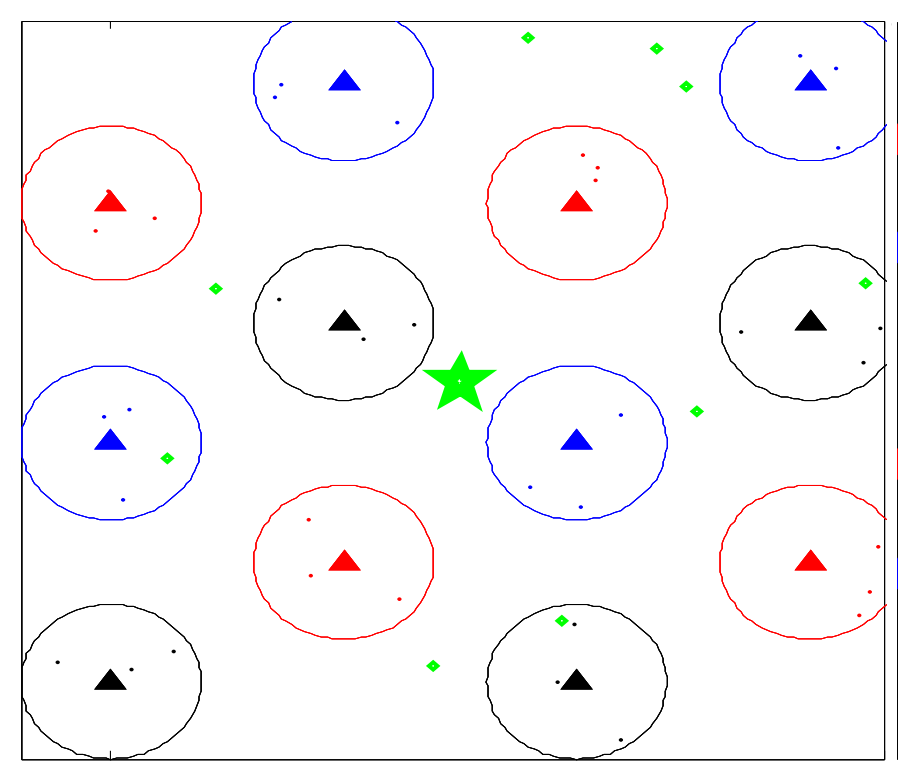

(a)

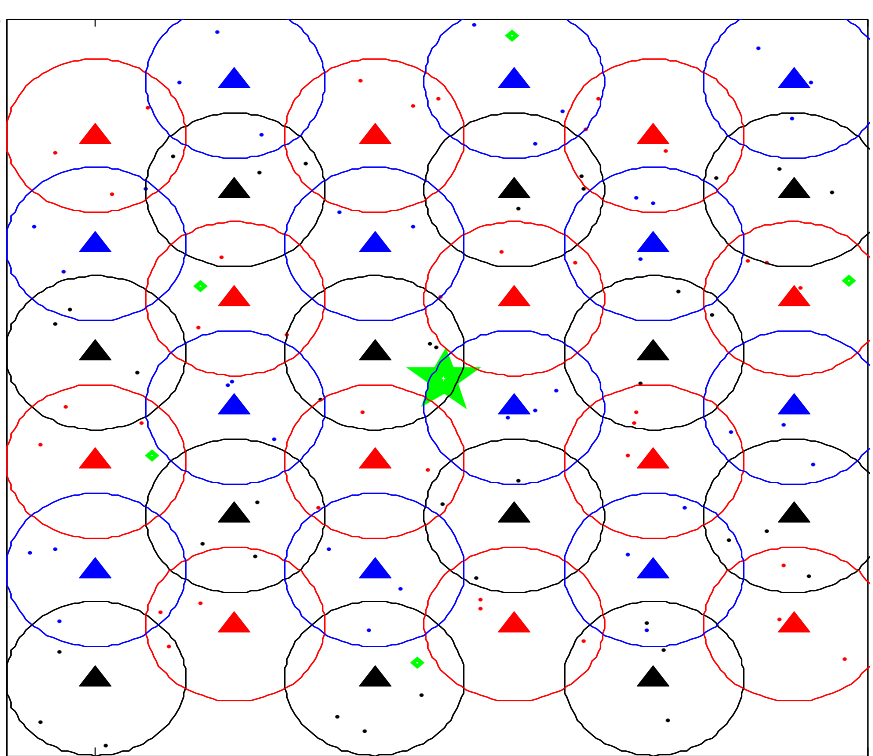

(b)

Figure A.1: Organized HetNet: (a) low density network, (b) dense network. Green star: MBS

Green dots: MUs

Coloured triangles: SBSs

Small coloured dots: SCUs

\section{Random topology:}

As with organized topology, the MBS is installed in the centre of area $\mathcal{A}$, and its associated MUs are deployed in the area following a uniform random distribution. On the other hand, the SBSs are now deployed within $\mathcal{A}$, randomly following the Poisson Point Process (PPP). The SCUs are deployed with each small cell following a uniform random distribution. Fig. A.2 shows an example of a randomly deployed network. All small cells are the same colour as they all have full access to the spectrum.

\section{A.2 Channel realizations}

In both topologies, after picking the location of each node, the distance between each transmitter and receiver pair is calculated. Accordingly, the path loss on every link is 


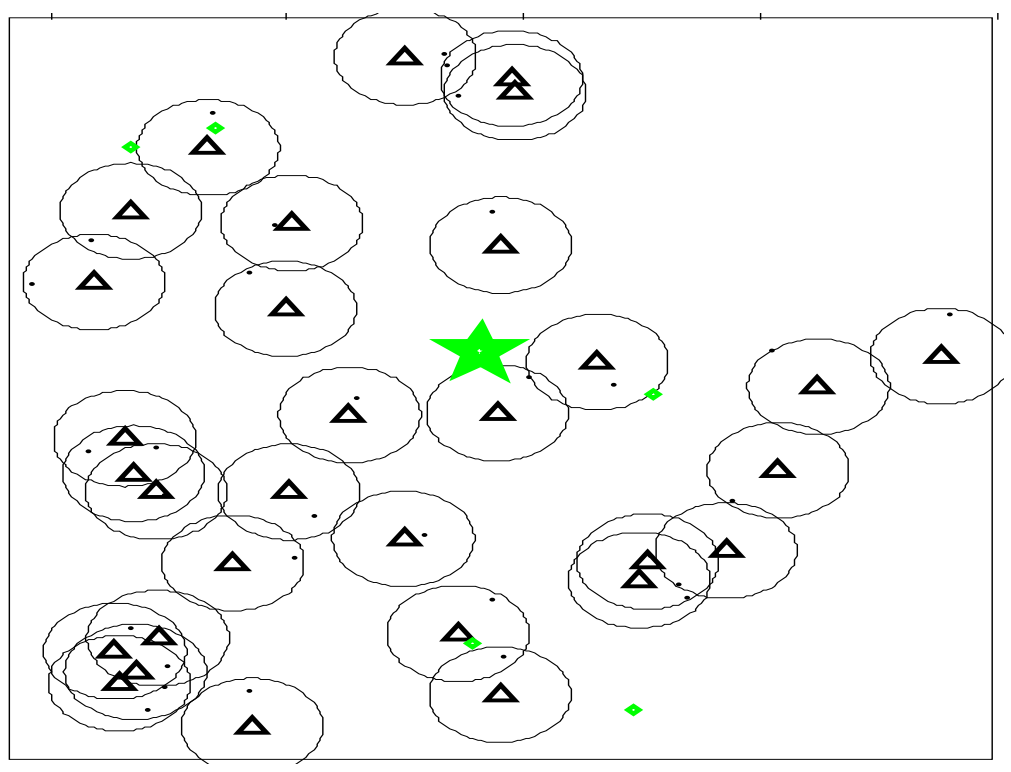

Figure A.2: HetNet system with random topology.

Green star: MBS

Green dots: MUs

Black triangles: SBSs

Small black dots: SCUs

found using the path loss model discussed in Section 2.1. Gaussian distributed shadow fading is then added (in dB) to the path loss effect for each link. Subsequently, a fading channel realization for each link is created over both dimensions (time and frequency) to capture the small scale fading, which is modelled using the Rayleigh distribution, as discussed in Section 2.1. In the whole thesis, we consider block fading channels in which the gain of every channel will be flat during each time slot. Fig. A.3.a \& b show two examples of time-frequency fading realizations for a desired link and an interfering link respectively.

After creating the realizations for all the links, these data are fed to the systems that we would like to examine (i.e. the proposed and the benchmark systems). 


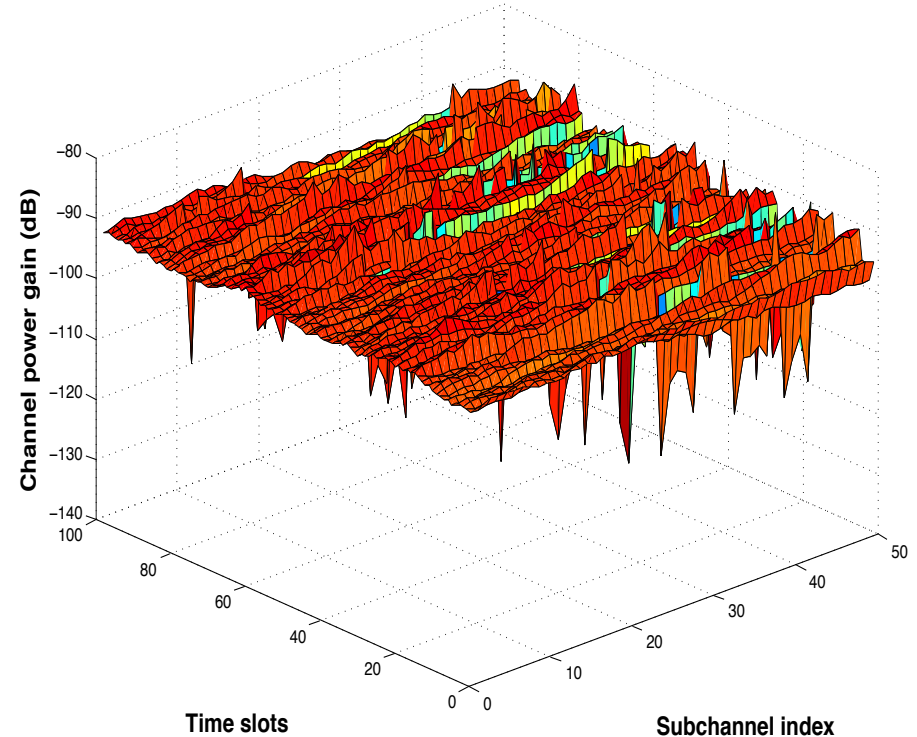

(a)

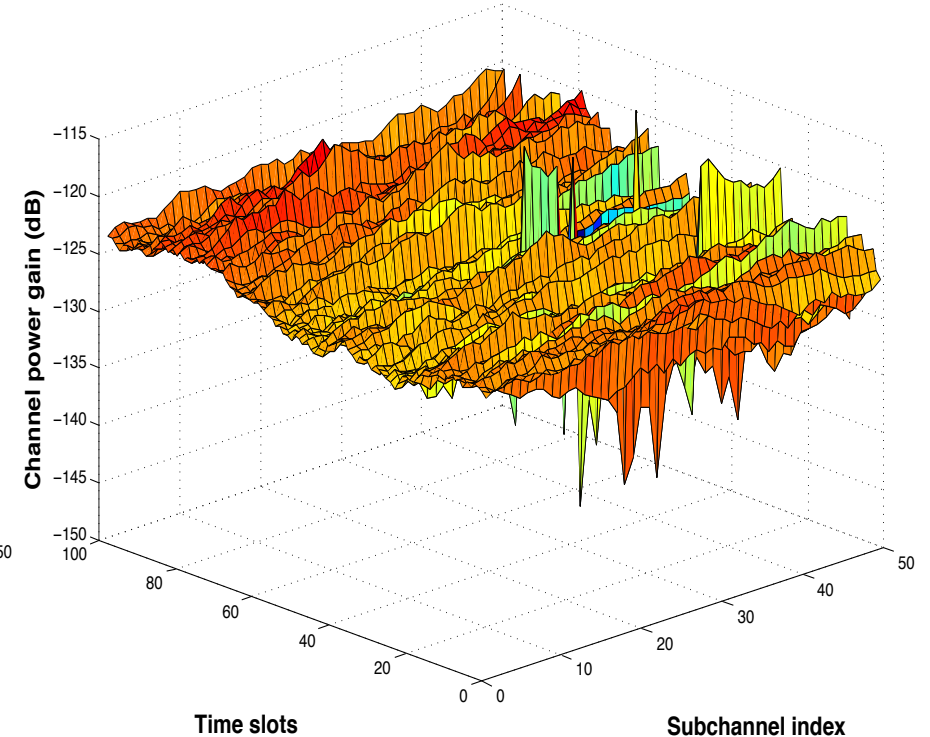

(b)

Figure A.3: Time-frequency fading realizations: (a) desired link, (b) interfering link. 